\title{
Fermion mass hierarchies from vectorlike families with an extended 2HDM and a possible explanation for the electron and muon anomalous magnetic moments
}

\author{
A. E. Cárcamo Hernández $\oplus^{1, *}$ S. F. King $\oplus^{2, \dagger}$ and H. Lee $\oplus^{2, \$}$ \\ ${ }^{1}$ Departamento de Física, Universidad Técnica Federico Santa María, \\ Casilla 110-V, Valparaíso, Chile \\ ${ }^{2}$ School of Physics and Astronomy, University of Southampton, \\ SO17 1BJ Southampton, United Kingdom
}

(Received 23 January 2021; accepted 6 May 2021; published 22 June 2021)

\begin{abstract}
We study an extended 2 Higgs doublet model (2HDM) in which the Standard Model (SM) Yukawa interactions are forbidden due to a global $U(1)^{\prime}$ symmetry, but may arise via mixing with vectorlike families. In this model, the hierarchical structure of Yukawa couplings of quarks and leptons in the SM arises from the heavy masses of the fourth and fifth vectorlike families. Within this model, we consider various nonstandard contributions to the electron and muon anomalous magnetic moments. We first consider the $W$ exchange at one-loop level, consistent with the $\mu \rightarrow e \gamma$ constraint, and show that it yields a negligible contribution to both electron and muon anomalous magnetic moments. We then consider Higgs scalar exchange, together with vectorlike leptons, at one-loop level and show that it is possible to have nonstandard contributions to the electron and muon anomalous magnetic moments within the $1 \sigma$ constraint of certain experiments. We present some benchmark points for both the muon and the electron anomalies, together with some numerical scans around these points, which indicate the mass regions of the Higgs scalars of the $2 \mathrm{HDM}$ in this scenario.
\end{abstract}

DOI: 10.1103/PhysRevD.103.115024

\section{INTRODUCTION}

The Standard Model (SM) has made many successful predictions for the phenomenology of both quark and lepton sectors with very high accuracy. However there are long-established anomalies which are not addressed by the SM such as muon and electron anomalous magnetic moments $a_{\mu}=(g-2)_{\mu} / 2, a_{e}=(g-2)_{e} / 2$. The muon anomalous magnetic moment reported by the Brookhaven E821 experiment at BNL [1] and the electron anomaly have confirmed $+3.5 \sigma$ and $-2.5 \sigma$ deviations from the SM, respectively. Detailed data analysis of the Standard Model predictions for the muon anomalous magnetic moment are provided in [2-6]. The experimentally observed values for the muon and electron

\footnotetext{
*antonio.carcamo@usm.cl

king@soton.ac.uk

"hl2n18@soton.ac.uk
}

Published by the American Physical Society under the terms of the Creative Commons Attribution 4.0 International license. Further distribution of this work must maintain attribution to the author(s) and the published article's title, journal citation, and DOI. Funded by SCOAP. anomalies at $1 \sigma$ of experimental error bars, respectively, $\operatorname{read}^{1}$ :

$$
\begin{aligned}
& \Delta a_{\mu}=a_{\mu}^{\mathrm{Exp}}-a_{\mu}^{\mathrm{SM}}=(26.1 \pm 8.0) \times 10^{-10} \\
& \Delta a_{e}=a_{e}^{\mathrm{Exp}}-a_{e}^{\mathrm{SM}}=(-0.88 \pm 0.36) \times 10^{-12} .
\end{aligned}
$$

When trying to explain both anomalies to within $1 \sigma$, a main difficulty arises from the sign of each anomaly: the muon anomaly requires positive definite nonstandard contributions, whereas the electron anomaly requires such contributions to contribute with a negative sign [8]. Without loss of generality, the Feynman diagrams corresponding to the contributions for the muon and electron anomalies take the same internal structure at one-loop

\footnotetext{
${ }^{1}$ It is worth mentioning that the experimental value of the anomalous magnetic moment of the electron is sensitive to the measurement of the fine-structure constant $\alpha$. The experimental value of $\Delta a_{e}=a_{e, \text { exp }}-a_{e}\left(\alpha_{\text {Berkeley }}\right)$ used in this work and given in Eq. (1) is obtained using $\alpha_{\text {Berkeley }}$ from caesium recoil measurements by the Berkeley 2018 experiment [7]. As this paper was being completed a different experiment [8] reported a result that implies $\Delta a_{e}=a_{e}^{\text {Exp }}-a_{e}^{\mathrm{SM}}=(0.48 \pm 0.30) \times 10^{-12}$ which differs from the SM by $+1.6 \sigma$. The two experiments appear to be inconsistent with each other, and our results here are based on the earlier result in Eq. (1).
} 

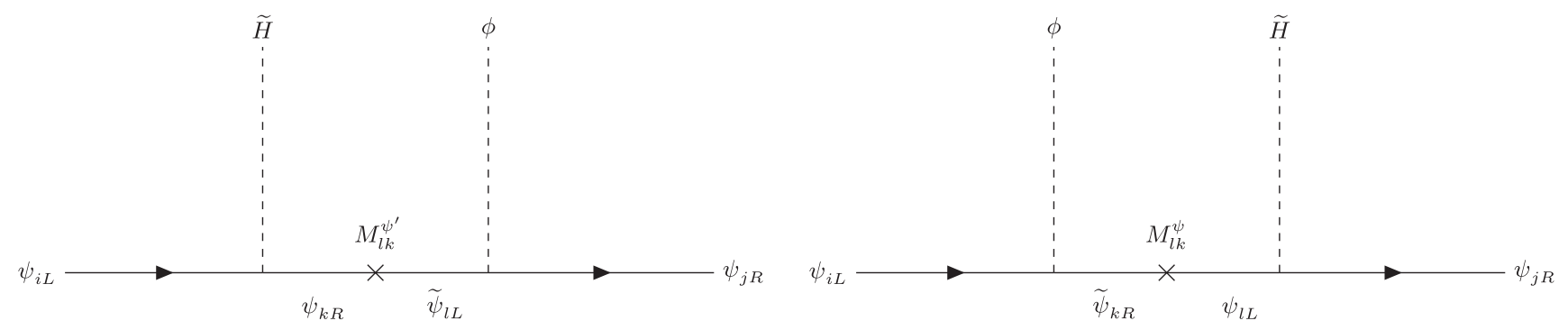

FIG. 1. Diagrams in this model which lead to the effective Yukawa interactions, where $\psi, \psi^{\prime}=Q, u, d, L, e$ (neutrinos will be treated separately) $i, j=1,2,3, k, l=4,5, M_{l k}$ is vectorlike mass and $\tilde{H}=i \sigma_{2} H^{*}, H=H_{u, d}$

except from the fact that the external particles are different. The similar structure of the one-loop level contributions to the muon and electron anomalous magnetic moments might be able to be explained by the same new physics, but accounting for the relative negative sign is challenging. For example, considering the one-loop exchange of $W$ or $Z^{\prime}$ gauge bosons results in theoretical predictions for the muon and electron anomalies having the same sign.

In this paper we take the view that both anomalies should be explained to $1 \sigma$ using the same internal structure at the one-loop level by some new physics which is capable of accounting for the correct signs of the anomalies. To explain the muon and electron anomalies, we focus on a well motivated model which is also capable of accounting for origin of Yukawa couplings and hierarchies in the SM. The model we consider will account for the Yukawa coupling constant for the top quark being nearly 1 while that for the electron is around $10^{-6}$, as well as all the other fermion hierarchies in between, as well as the neutrino masses and mixing. In order to achieve this we shall introduce vectorlike particles, which are charged under a global $U(1)^{\prime}$ symmetry. In a related previous work [9], with a gauged $U(1)^{\prime}$ symmetry, the first family of quarks and leptons remained massless when only one vectorlike family is included. Here we shall modify the model to include two vectorlike families charged under a global $U(1)^{\prime}$ to allow also the first family to be massive and avoid $Z^{\prime}$ constraints. Then we shall apply the resulting model to the problem of muon and electron anomalous magnetic moments. The considered model is based on a 2 Higgs doublet model (2HDM) extension of the SM, supplemented by a global $U(1)^{\prime}$ symmetry, where the particle spectrum is enlarged by the inclusion of two vectorlike fermion families, as well as one singlet Higgs to break the $U(1)^{\prime}$ symmetry. ${ }^{2}$ The SM Yukawa interactions are forbidden, but the Yukawa interactions with vectorlike families charged under the $U(1)^{\prime}$ symmetry are allowed. Once the flavon develops a vev and the heavy vectorlike fermions are integrated out, the

\footnotetext{
${ }^{2}$ An example of a multi-Higgs doublet model that uses a flavor dependent global $U(1)^{\prime}$ symmetry to explain the SM charged fermion mass hierarchy by hierarchies of the vacuum expectation values of the Higgs doublets is provided in [10].
}

effective SM Yukawa interactions are generated, as indicated in Fig. 1. Furthermore, this model also highlights the shape of the 2HDM model type II, since in our proposed model, one Higgs doublet (which in the alignment limit corresponds to the SM Higgs doublet) couples with the up type quarks whereas the other one features Yukawa interactions with down type quarks and SM charged leptons. Regarding the neutrino sector, since we consider the SM neutrinos as Majorana particles, we have that this sector requires another approach relying on the inclusion of a new five dimensional Weinberg-like operator, which is allowed in this model and which requires both SM Higgs doublets to be present, namely the so called type Ib seesaw model [11].

We shall show that the heavy vectorlike leptons are useful and necessary to explain the anomalous electron and muon magnetic moment deviations from the SM, of magnitude and opposite signs given in Eq. (1). A study of such $g-2$ anomalies in terms of new physics and a possible UV complete explanation via vectorlike leptons was performed in [12], although the model presented here is quite different, since our model is motivated by the requirement of accounting also for the fermion mass hierarchies. Other theories with extended symmetries and particle spectrum have also been proposed to find an explanation for the muon and electron anomalous magnetic moments [12-58]. In the following we provide a brief comparison of our model to other works, starting with the model proposed in [49] where vectorlike leptons are also present. The model of [49] corresponds to an extended type $X$ lepton specific $2 \mathrm{HDM}$ model of [49] having a $Z_{2}$ discrete symmetry under which one of the scalar doublets and the leptonic fields are charged. In such model the vectorlike leptons induces a one-loop level contribution to the electron anomalous magnetic moment whereas the muon anomalous magnetic moment is generated at two-loop via the exchange of a light pseudoscalar. On the other hand, in our proposed model a spontaneously broken global $U(1)^{\prime}$ symmetry is considered instead of the $Z_{2}$ symmetry and the vectorlike leptons generate one-loop level contributions to the muon and electron anomalous magnetic moments and at the same type produce the SM charged lepton masses, thus providing a connection of the charged lepton 
mass generation mechanism and the $g-2$ anomalies, which is not given in the model of [49]. It is also worth emphasizing that our model is very different from other models proposed in the literature based on the Universal Seesaw mechanism [59-76]. Universal Seesaw models are typically based on the left-right symmetric model with electroweak singlet fermions only, while our vectorlike fermions involves complete families, including electroweak doublets which are typically the lightest ones. Some examples of theories relying on the Universal Seesaw mechanism to explain the SM charged fermion mass hierarchy are provided in [59-76].

In the approach followed in this paper the large third family quark and lepton Yukawa couplings are effectively generated via mixing with a vectorlike fourth family of electroweak doublet fermions, which are assumed to be relatively light, with masses around the $\mathrm{TeV}$ scale. The smallness of the second family quark and lepton Yukawa couplings is due to their coupling to heavier vectorlike fourth family electroweak singlet fermions. Similar considerations apply to the lightest first family quarks and leptons which couple to heavy fifth family vectorlike fermions. It may seem that the problem of the hierarchies of SM fermions is not solved but simply reparametrized in terms of unknown vectorlike fermion masses. However, there are four advantages to this approach. First, the approach is dynamical, since the vectorlike masses are new physical quantities which could in principle be determined by a future theory. Second, it has experimental consequences, since the new vectorlike fermions can be discovered either directly, or (as in this paper) indirectly via their loop contributions. Third, this approach can also account for small quark mixing angles [9], as well as large lepton mixing angles via the type $\mathrm{Ib}$ seesaw mechanism [11]. Fourth, the effective Yukawa couplings are proportional to a product of two other dimensionless couplings, so a small hierarchy in those couplings can give a quadratically larger hierarchy in the effective couplings. For all these reasons, the approach we follow in this paper is both well motivated and interesting.

Returning to our proposed model framework, we first consider the contribution of $W$ boson exchange with neutrinos to the electron and muon anomalous magnetic moments at the one-loop level. Since this model involves the vectorlike neutrinos, the sensitivity of the branching ratio of $\mu \rightarrow e \gamma$ decay can be enhanced with respect to the observable level and the muon and electron anomalous magnetic moments are studied while keeping the $\mu \rightarrow e \gamma$ constraint. As a result, we find that the impact of our predictions with $W$ exchange at one-loop level is negligible when compared to their experimental bound. We then consider the contributions from the 2HDM scalar exchange. To study the implications of the one-loop level scalar exchange in the muon and electron anomalous magnetic moments, we first construct a scalar potential and derive the mass squared matrix for $C P$-even, $C P$-odd, and charged Higgses assuming there is no mixing between the SM Higgs $h$ and two non-SM physical scalars $H_{1,2}$. A diagonal Yukawa matrix for charged leptons implies the absence of mixing between charged leptons, resulting in vanishing branching ratio for the $\mu \rightarrow e \gamma$ decay, which in turn leads to a fulfillment of the charged lepton flavor violating constraints in this scenario. In such a framework we show that both anomalies can successfully explain both anomalies, including their opposite signs, at the $1 \sigma$ level. We present some benchmark points for both the muon and the electron anomalies, together with some numerical scans around these points, which indicate the mass regions of the Higgs scalars of the $2 \mathrm{HDM}$ in this scenario. We also provide some analytic arguments to augment the numerical results.

The layout of the remainder of the paper is as follows. In Sec. II we discuss the origin of Yukawa couplings from a fourth and fifth vectorlike family, within a mass insertion formalism. In Sec. III we construct the effective Yukawa matrices using a more detailed mixing formalism which goes beyond the mass insertion formalism. In Sec. IV we consider $W$ exchange contributions to $(g-2)_{\mu},(g-2)_{e}$ and $\operatorname{BR}(\mu \rightarrow e \gamma)$ based on the type Ib seesaw mechanism within our model and show that the contributions are too small. In Sec. V we turn to Higgs scalar exchange contributions to $(g-2)_{\mu},(g-2)_{e}$ and $\operatorname{BR}(\mu \rightarrow e \gamma)$, focussing on analytical formulas. Then in Sec. VI we give a full numerical analysis of such contributions, showing that they can successfully explain the anomalies, presenting some benchmark points for both the muon and the electron anomalies, together with some numerical scans around these points, which indicate the mass regions of the Higgs scalars of the 2HDM in this scenario. Section VII concludes the main body of the paper. Appendix A provides a discussion of the quark mass matrices in two bases. Appendix B includes a brief discussion of heavy scalar production at a proton-proton collider.

\section{THE ORIGIN OF YUKAWA COUPLINGS FROM A FOURTH AND FIFTH VECTORLIKE FAMILY}

We start by asking a question: what is the origin of the SM Yukawa couplings? In addressing such question, we assume that the SM Yukawa Lagrangian is the low energy limit of an extended theory with enlarged symmetry and particle spectrum, and arises after the spontaneous breaking of an $U(1)^{\prime}$ global symmetry at an energy scale as low as $\mathrm{TeV}$. Therefore, understanding the origin of the Yukawa interaction naturally leads to the presence of another Higgses whose masses are higher than the mass of the SM Higgs. Furthermore, the SM Yukawa interactions are forbidden by the global $U(1)^{\prime}$ symmetry, however the Yukawa interaction with the vectorlike particles are allowed. With these considerations in place, the possible 
diagrams generating the Yukawa interactions can be drawn as indicated in Fig. 1.

There are two key features in Fig. 1, one of which is the presence of the assumed flavon $\phi$ and the other one is the vectorlike mass $M$. Once the flavon $\phi$ develops its vev, the effective Yukawa interactions $\bar{\psi}_{i L} \tilde{H} \psi_{j R}$ are generated with a coupling constant proportional to $\langle\phi\rangle / M$, assumed to be less than unity, which appears in front of the usual Yukawa constant. The proportional factor $\langle\phi\rangle / M$ plays a crucial role in explaining why one Yukawa constant can be relatively smaller or bigger than the other ones since the magnitude of each Yukawa constant is accompanied by the mass of the vectorlike particles. The effective Lagrangian in this diagram reads in the mass insertion formalism:

$$
\begin{aligned}
\mathcal{L}_{\text {eff }}^{\text {Yukawa }}= & y_{i k}^{\psi}\left(M_{\psi^{\prime}}^{-1}\right)_{k l} x_{l j}^{\psi^{\prime}}\langle\phi\rangle \bar{\psi}_{i L} \tilde{H} \psi_{j R} \\
& +x_{i k}^{\psi}\langle\phi\rangle\left(M_{\psi}^{-1}\right)_{k l} y_{l j}^{\psi} \bar{\psi}_{i L} \tilde{H} \psi_{j R}+\text { H.c. }
\end{aligned}
$$

where $\psi, \psi^{\prime}=Q, u, d, L, e$ (neutrinos will be treated separately) and $x$ is a Yukawa constant in the interaction with $\phi$ and $y$ is in the interaction with $\tilde{H}$ as per Fig. 1 . Throughout this work, we take a view that the Yukawa constant $y$ can be ideally of order unity while the $x$ is small compared to the $y$. We shall also use a mixing formalism rather than the mass insertion formalism.

\section{A. The model with $\boldsymbol{U}(1)^{\prime}$ global symmetry}

For an analysis of the phenomenology described above, we extend the SM fermion sector by adding two vectorlike fermions, the SM gauge symmetry by including the global $U(1)^{\prime}$ symmetry and the scalar sector of the 2HDM model is enlarged by considering a gauge scalar singlet, whose VEV triggers the spontaneous breaking of the $U(1)^{\prime}$ symmetry. The scalar sector of the model is composed of by two $S U(2)$ doublet scalars $H_{u, d}$ and one flavon $\phi$. Our extended 2HDM with enlarged particle spectrum and symmetries has the interesting feature that the SM Yukawa interactions are forbidden due to the global $U(1)^{\prime}$ symmetry whereas the Yukawa interactions of SM fermions with vectorlike families are allowed. Furthermore, such vectorlike families have mass terms which are allowed by the symmetry. Thus, the SM charged fermions masses are generated from a universal seesaw mechanism mediated by heavy vectorlike fermions. Unlike the $U(1)^{\prime}$ model proposed in [77], we assume that the $U(1)^{\prime}$ symmetry is global instead of local. This allows us more flexibility in the allowed range for the scale where the $U(1)^{\prime}$ symmetry is broken. On top of that, the up-type quarks feature Yukawa interaction with the up-type Higgs whereas the down-type ones interact with down-type Higgs. In this BSM model, the SM particles are neutral under the $U(1)^{\prime}$ symmetry, while the vectorlike particles and all other scalars are charged under the symmetry. The particle content and symmetries of the model are shown in Table I.

The right-handed neutrinos $\nu_{i R}$ are absent in this model since we treat the left-handed neutrinos in the lepton doublet as Majorana particles and they are only extended by vectorlike neutrinos. The vectorlike particles and their partners have exact opposite charge to each other under the extended gauge symmetry to cancel out chiral anomaly. Lastly, the SM Higgses $H_{u, d}$ are negatively charged under the $U(1)^{\prime}$ symmetry to forbid the renormalizable SM Yukawa interactions.

\section{B. Mass insertion formalism}

The renormalizable Yukawa interactions and mass terms for both up and down quark sectors read:

$$
\begin{aligned}
\mathcal{L}_{q}^{\text {Yukawa }+ \text { Mass }}= & y_{i k}^{u} \bar{Q}_{i L} \tilde{H}_{u} u_{k R}+x_{k i}^{u} \phi \overline{\tilde{u}}_{k L} u_{i R}+x_{i k}^{Q} \phi \bar{Q}_{i L} \tilde{Q}_{k R} \\
& +y_{k i}^{u} \bar{Q}_{k L} \tilde{H}_{u} u_{i R}+y_{i k}^{d} \bar{Q}_{i L} \tilde{H}_{d} d_{k R} \\
& +x_{k i}^{d} \phi \overline{\tilde{d}}_{k L} d_{i R}+y_{k i}^{d} \bar{Q}_{k L} \tilde{H}_{d} d_{i R} \\
& +M_{k l}^{u} \overline{\tilde{u}}_{l L} u_{k R}+M_{k l}^{d} \overline{\tilde{d}}_{l L} d_{k R} \\
& +M_{k l}^{Q} \bar{Q}_{k L} \tilde{Q}_{l R}+\text { H.c. }
\end{aligned}
$$

where $i, j=1,2,3, k, l=4,5$ and $\tilde{H}=i \sigma_{2} H^{*}$. The possible diagrams contributing to the low energy quark Yukawa interaction are given in Fig. 2:

The above two diagrams correspond to the up-type quark sector whereas the below two diagrams correspond to the down-type quark sector. The model under consideration is an extended 2HDM where the up-type Higgs $H_{u}$ is relevant

TABLE I. This model is an extended $2 \mathrm{HDM}$ by the global $U(1)^{\prime}$ symmetry with two vectorlike families plus one flavon and reflects the property that the SM Yukawa interactions are forbidden. All SM particles $\psi_{i}(i=1,2,3)$ are neutral under the $U(1)^{\prime}$ symmetry and the right neutrinos $\nu_{i R}$ are not considered. Notice that this model involves two right-handed vectorlike neutrinos $\nu_{k R}, \widetilde{\nu}_{k R}$. The SM particles are extended by two vectorlike families where $k=4,5$ and two SM Higgses $H_{u, d}$ are charged negatively under $U(1)^{\prime}$ to forbid the renormalizable SM Yukawa interactions. The flavon field $\phi$ plays a role of breaking the $U(1)^{\prime}$ symmetry at TeV scale.

\begin{tabular}{lcccrcccccccccccccccc}
\hline \hline Field & $Q_{i L}$ & $u_{i R}$ & $d_{i R}$ & $L_{i L}$ & $e_{i R}$ & $Q_{k L}$ & $u_{k R}$ & $d_{k R}$ & $L_{k L}$ & $e_{k R}$ & $\nu_{k R}$ & $\tilde{Q}_{k R}$ & $\tilde{u}_{k L}$ & $\tilde{d}_{k L}$ & $\tilde{L}_{k R}$ & $\tilde{e}_{k L}$ & $\tilde{\nu}_{k R}$ & $\phi$ & $H_{u}$ & $H_{d}$ \\
\hline$S U(3)_{C}$ & $\mathbf{3}$ & $\mathbf{3}$ & $\mathbf{3}$ & $\mathbf{1}$ & $\mathbf{1}$ & $\mathbf{3}$ & $\mathbf{3}$ & $\mathbf{3}$ & $\mathbf{1}$ & $\mathbf{1}$ & $\mathbf{1}$ & $\mathbf{3}$ & $\mathbf{3}$ & $\mathbf{3}$ & $\mathbf{1}$ & $\mathbf{1}$ & $\mathbf{1}$ & $\mathbf{1}$ & $\mathbf{1}$ & $\mathbf{1}$ \\
$S U(2)_{L}$ & $\mathbf{2}$ & $\mathbf{1}$ & $\mathbf{1}$ & $\mathbf{2}$ & $\mathbf{1}$ & $\mathbf{2}$ & $\mathbf{1}$ & $\mathbf{1}$ & $\mathbf{2}$ & $\mathbf{1}$ & $\mathbf{1}$ & $\mathbf{2}$ & $\mathbf{1}$ & $\mathbf{1}$ & $\mathbf{2}$ & $\mathbf{1}$ & $\mathbf{1}$ & $\mathbf{1}$ & $\mathbf{2}$ & $\mathbf{2}$ \\
$U(1)_{Y}$ & $\frac{1}{6}$ & $\frac{2}{3}$ & $-\frac{1}{3}$ & $-\frac{1}{2}$ & 1 & $\frac{1}{6}$ & $\frac{2}{3}$ & $-\frac{1}{3}$ & $-\frac{1}{2}$ & -1 & 0 & $\frac{1}{6}$ & $\frac{2}{3}$ & $-\frac{1}{3}$ & $-\frac{1}{2}$ & -1 & 0 & 0 & $\frac{1}{2}$ & $-\frac{1}{2}$ \\
$U(1)^{\prime}$ & 0 & 0 & 0 & 0 & 0 & 1 & -1 & -1 & 1 & -1 & -1 & 1 & -1 & -1 & 1 & -1 & -1 & 1 & -1 & -1 \\
\hline \hline
\end{tabular}



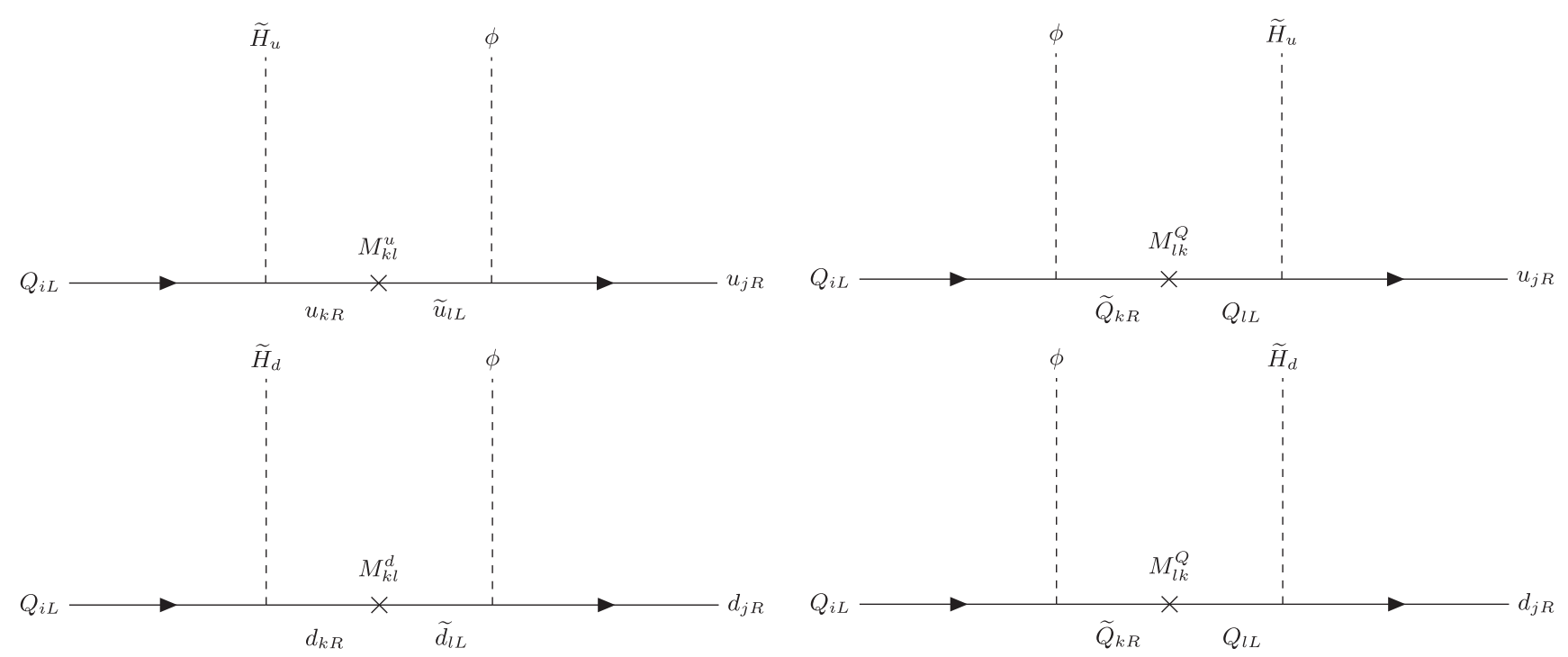

FIG. 2. Diagrams in this model which lead to the effective Yukawa interactions for the up quark sector(two above diagrams) and the down quark sector(two below diagrams) in mass insertion formalism, where $i, j=1,2,3$ and $k, l=4,5$ and $M_{l k}$ is vectorlike mass.

for the up-type quark sector whereas the down-type Higgs $H_{d}$ is suitable for the down-type quark and charged lepton sectors. Like in the quark sector, the Yukawa interactions and mass terms for charged leptons can be written in a similar way:

$$
\begin{aligned}
\mathcal{L}_{e}^{\text {Yukawa }+ \text { Mass }}= & y_{i k}^{e} \bar{L}_{i L} \tilde{H}_{d} e_{k R}+x_{k i}^{e} \phi \overline{\tilde{e}}_{k L} e_{i R}+x_{i k}^{L} \phi \bar{L}_{i L} \tilde{L}_{k R} \\
& +y_{k i}^{e} \bar{L}_{k L} \tilde{H}_{d} e_{i R}+M_{k l}^{e} \tilde{\tilde{e}}_{l L} e_{k R}+M_{k l}^{L} \bar{L}_{k L} \tilde{L}_{l R} \\
& + \text { H.c. }
\end{aligned}
$$

Then, the possible diagrams giving rise to the charged lepton Yukawa interactions are shown in Fig. 3:

As for the neutrinos, its behavior is different as compared to the quarks or charged leptons since there exists only Majorana neutrinos in this model so initial and final neutrinos in mass insertion formalism diagrams must be same. The Yukawa interactions and mass terms for the neutrino sector are given by:

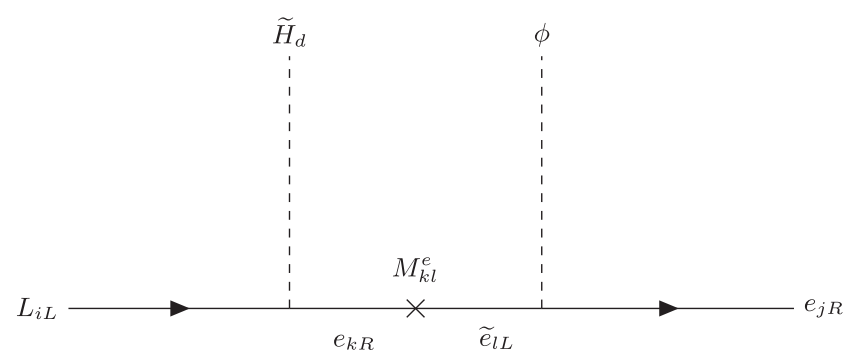

$$
\begin{aligned}
\mathcal{L}_{\nu}^{\text {Yukawa+Mass }}= & y_{i k}^{\nu} \bar{L}_{i L} \tilde{H}_{u} \nu_{k R}+x_{i k}^{L} \bar{L}_{i L} H_{d} \overline{\tilde{\nu}}_{k R}+M_{k l}^{M} \overline{\tilde{\nu}}_{l R} \nu_{k R} \\
& + \text { H.c. }
\end{aligned}
$$

Here, one important feature in Eq. (5) is the presence of the vectorlike mass $M$. From the two Yukawa interactions in Eq. (5), it follows that both $\nu_{R}$ and $\tilde{\nu}_{R}$ have a lepton number +1 and they are different particles. And then taking a look at the vectorlike mass term in Eq. (5), it can be confirmed that the vectorlike mass is not a strict Majorana mass because $\nu_{R}$ and $\tilde{\nu}_{R}$ are different particles but plays a role of Majorana mass since the mass term violates the lepton number conservation. The corresponding diagram for the neutrino sector in the mass insertion formalism is given in Figure 4. However for our calculations we use a mixing formalism (see next section).

The operator $\bar{L}_{i} \bar{L}_{j} \tilde{H}_{u} H_{d}$ resulting from Fig. 4 gives rise to the so called type Ib seesaw mechanism [11] which differs from the usual type Ia seesaw mechanism corresponding to the Weinberg operator $\bar{L}_{i} \bar{L}_{j} \tilde{H}_{u} \tilde{H}_{u}$ and will be discussed later in detail.

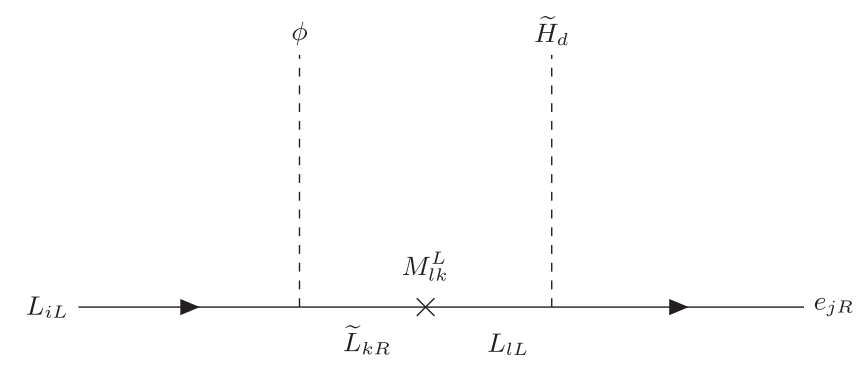

FIG. 3. Diagrams in this model which lead to the effective Yukawa interactions for the charged lepton sector in mass insertion formalism, where $i, j=1,2,3$ and $k, l=4,5$ and $M_{l k}$ is vectorlike mass. 


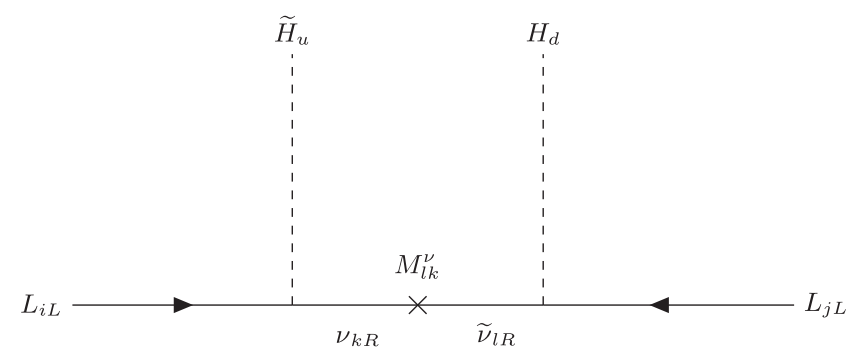

FIG. 4. Type Ib seesaw diagram [11] which leads to the effective Yukawa interactions for the Majorana neutrinos in mass insertion formalism, where $i, j=1,2,3$ and $k, l=4,5$ and $M_{l k}$ is vectorlike mass.

\section{EFFECTIVE YUKAWA MATRICES USING A MIXING FORMALISM}

As seen from Eq. (2), we need to mix Higgses with the flavon to generate the effective Yukawa Lagrangian required to produce the SM fermion mass hierarchy. Since there is no an extra symmetry or constraint to keep the mixing between Higgses and flavon from taking place, it is natural to assume their mixing.

\section{A. The $7 \times 7$ matrix}

Consider the $7 \times 7$ mass matrix for Dirac fermions:

$$
M^{\psi}=\left(\begin{array}{c|ccccccc} 
& \psi_{1 R} & \psi_{2 R} & \psi_{3 R} & \psi_{4 R} & \psi_{5 R} & \tilde{\psi}_{4 R} & \tilde{\psi}_{5 R} \\
\hline \bar{\psi}_{1 L} & 0 & 0 & 0 & y_{14}^{\psi}\left\langle\tilde{H}^{0}\right\rangle & y_{15}^{\psi}\left\langle\tilde{H}^{0}\right\rangle & x_{14}^{\psi}\langle\phi\rangle & x_{15}^{\psi}\langle\phi\rangle \\
\bar{\psi}_{2 L} & 0 & 0 & 0 & y_{24}^{\psi}\left\langle\tilde{H}^{0}\right\rangle & y_{25}^{\psi}\left\langle\tilde{H}^{0}\right\rangle & x_{24}^{\psi}\langle\phi\rangle & x_{25}^{\psi}\langle\phi\rangle \\
\bar{\psi}_{3 L} & 0 & 0 & 0 & y_{34}^{\psi}\left\langle\tilde{H}^{0}\right\rangle & y_{35}^{\psi}\left\langle\tilde{H}^{0}\right\rangle & x_{34}^{\psi}\langle\phi\rangle & x_{35}^{\psi}\langle\phi\rangle \\
\bar{\psi}_{4 L} & y_{41}^{\psi}\left\langle\tilde{H}^{0}\right\rangle & y_{42}^{\psi}\left\langle\tilde{H}^{0}\right\rangle & y_{43}^{\psi \prime}\left\langle\tilde{H}^{0}\right\rangle & 0 & 0 & M_{44}^{\psi} & M_{45}^{\psi} \\
\bar{\psi}_{5 L} & y_{51}^{\psi}\left\langle\tilde{H}^{0}\right\rangle & y_{52}^{\psi}\left\langle\tilde{H}^{0}\right\rangle & y_{53}^{\psi}\left\langle\tilde{H}^{0}\right\rangle & 0 & 0 & M_{54}^{\psi} & M_{55}^{\psi} \\
\overline{\tilde{\psi}}_{4 L} & x_{41}^{\psi^{\prime}}\langle\phi\rangle & x_{42}^{\psi^{\prime}}\langle\phi\rangle & x_{43}^{\psi^{\prime}}\langle\phi\rangle & M_{44}^{\psi^{\prime}} & M_{45}^{\psi^{\prime}} & 0 & 0 \\
\overline{\tilde{\psi}}_{5 L} & x_{51}^{\psi^{\prime}}\langle\phi\rangle & x_{52}^{\psi^{\prime}}\langle\phi\rangle & x_{53}^{\psi^{\prime}}\langle\phi\rangle & M_{54}^{\psi^{\prime}} & M_{55}^{\psi^{\prime}} & 0 & 0
\end{array}\right),
$$

with the coefficients $y$ and $x$ being Yukawa constants where the former is expected to be of order unity whereas the latter is smaller than $y$. Furthermore, the $125 \mathrm{GeV}$ SM like Higgs boson $H$ will corresponds to the lightest of the $C P$ even neutral scalar states arising from $H_{u}, H_{d}$, and $\phi$, whereas $M$ is the vectorlike mass. The column vector located at the lower left block in Eq. (6) consists of left-handed particles while the row vector at the upper right block are made up of right-handed particles. The zeros in the $3 \times 3$ upper block in Eq. (6) mean that no SM Yukawa interactions take place due to charge conservation as well as zeros in two $2 \times 2$ blocks. Since we are interested in explaining the muon and electron anomalous magnetic moments in this model, we first focus on the lepton sector in the next subsection and the method used for obtaining the low energy SM Yukawa matrices in the lepton sector can be applied to the quark sector in the same way with a slight change so that the quark sector will be discussed in Appendix A.

\section{B. A convenient basis for charged leptons}

From Eq. (6), we can take a specified basis by rotating some fields as below:

$$
M^{e}=\left(\begin{array}{c|ccccccc} 
& e_{1 R} & e_{2 R} & e_{3 R} & e_{4 R} & e_{5 R} & \tilde{L}_{4 R} & \tilde{L}_{5 R} \\
\hline \bar{L}_{1 L} & 0 & 0 & 0 & 0 & y_{15}^{e} v_{d} & 0 & x_{15}^{L} v_{\phi} \\
\bar{L}_{2 L} & 0 & 0 & 0 & y_{24}^{e} v_{d} & y_{25}^{e} v_{d} & 0 & x_{25}^{L} v_{\phi} \\
\bar{L}_{3 L} & 0 & 0 & 0 & y_{34}^{e} v_{d} & y_{35}^{e} v_{d} & x_{34}^{L} v_{\phi} & x_{35}^{L} v_{\phi} \\
\bar{L}_{4 L} & 0 & 0 & y_{43}^{e} v_{d} & 0 & 0 & M_{44}^{L} & M_{45}^{L} \\
\bar{L}_{5 L} & y_{51}^{e} v_{d} & y_{52}^{e} v_{d} & y_{53}^{e} v_{d} & 0 & 0 & 0 & M_{55}^{L} \\
\overline{\tilde{e}}_{4 L} & 0 & x_{42}^{e} v_{\phi} & x_{43}^{e} v_{\phi} & M_{44}^{e} & 0 & 0 & 0 \\
\overline{\tilde{e}}_{5 L} & x_{51}^{e} v_{\phi} & x_{52}^{e} v_{\phi} & x_{53}^{e} v_{\phi} & M_{54}^{e} & M_{55}^{e} & 0 & 0
\end{array}\right),
$$


where $v_{d}=\left\langle H_{d}^{0}\right\rangle$ and $\nu_{\phi}=\langle\phi\rangle$. We start by pointing out the reason why we take this specific basis for the charged leptons. The reason is that the strong hierarchical structure of the SM fermion Yukawa couplings can be implemented by the rotations with a simple assumption in this model to be specified below. In order to arrive from Eq. (6) to Eq. (7), we rotate the leptonic fields $L_{4 L}$ and $L_{5 L}$ to turn off $M_{54}^{L}$ and rotate $e_{4 R}$ and $e_{5 R}$ to turn off $M_{45}^{e}$. Then, we can rotate $L_{1 L}$ and $L_{3 L}$ to set $x_{14}^{L} v_{\phi}$ to zero and then rotate $L_{2 L}$ and $L_{3 L}$ to set $x_{24}^{L} v_{\phi}$ to zero. The same rotation can be applied to $e_{1 R, 2 R, 3 R}$ to set $y_{41,42}^{e} v_{d}$ to zero. Finally, we can further rotate $L_{1 L}$ and $L_{2 L}$ to switch off $y_{14}^{e} v_{d}$ and this rotation also goes for $e_{1 R, 2 R}$ to switch off $x_{41}^{e} v_{\phi}$. The above given mass matrix includes three distinct mass scales which are the vev $v_{d}$ of the neutral component of the Higgs doublet $H_{d}$, the vev $v_{\phi}$ of the flavon $\phi$ and the vectorlike masses $M$, whose orders of magnitude can be in principle be different. Therefore, the mass matrix will be diagonalized by the seesaw mechanism step-by-step instead of diagonalizing it at once. This mechanism is also known as universal seesaw, and was proposed for the first time, in the context of a leftright symmetric model in [59].

\section{A basis for decoupling heavy fourth and fifth vectorlike family}

As mentioned in the previous Sec. III B, the mass matrix in Eq. (7) involves three distinct mass scales $v_{d}$, $v_{\phi}$, and $M$ so it is possible to split this whole mass matrix by partial blocks to group mass terms with vev of $H_{d}$ as in Eq. (8)

$$
M^{e}=\left(\begin{array}{c|ccccc|cc} 
& e_{1 R} & e_{2 R} & e_{3 R} & e_{4 R} & e_{5 R} & \tilde{L}_{4 R} & \tilde{L}_{5 R} \\
\hline \bar{L}_{1 L} & 0 & 0 & 0 & 0 & y_{15}^{e} v_{d} & 0 & x_{15}^{L} v_{\phi} \\
\bar{L}_{2 L} & 0 & 0 & 0 & y_{24}^{e} v_{d} & y_{25}^{e} v_{d} & 0 & x_{25}^{L} v_{\phi} \\
\bar{L}_{3 L} & 0 & 0 & 0 & y_{34}^{e} v_{d} & y_{35}^{e} v_{d} & x_{34}^{L} v_{\phi} & x_{35}^{L} v_{\phi} \\
\bar{L}_{4 L} & 0 & 0 & y_{43}^{e} v_{d} & 0 & 0 & M_{44}^{L} & M_{45}^{L} \\
\bar{L}_{5 L} & y_{51}^{e} v_{d} & y_{52}^{e} v_{d} & y_{53}^{e} v_{d} & 0 & 0 & 0 & M_{55}^{L} \\
\hline \overline{\tilde{e}}_{4 L} & 0 & x_{42}^{e} v_{\phi} & x_{43}^{e} v_{\phi} & M_{44}^{e} & 0 & 0 & 0 \\
\overline{\tilde{e}}_{5 L} & x_{51}^{e} v_{\phi} & x_{52}^{e} v_{\phi} & x_{53}^{e} v_{\phi} & M_{54}^{e} & M_{55}^{e} & 0 & 0
\end{array}\right) \text {, }
$$

and then elements of the blocks involving $\phi$ can be rotated away to make those zeros by unitary mixing matrices of Eq. (10) as per Eq. (9):

$$
M^{e}=\left(\begin{array}{c|ccccccc} 
& e_{1 R} & e_{2 R} & e_{3 R} & e_{4 R} & e_{5 R} & \tilde{L}_{4 R} & \tilde{L}_{5 R} \\
\hline \bar{L}_{1 L} & & & & & & 0 & 0 \\
\bar{L}_{2 L} & & & & & & 0 & 0 \\
\bar{L}_{3 L} & & & \tilde{y}_{\alpha \beta}^{\prime e} v_{d} & & & 0 & 0 \\
\bar{L}_{4 L} & & & & & & \tilde{M}_{44}^{L} & M_{45}^{\prime L} \\
\bar{L}_{5 L} & & & & & & 0 & \tilde{M}_{55}^{L} \\
\overline{\tilde{e}}_{4 L} & 0 & 0 & 0 & \tilde{M}_{44}^{e} & 0 & 0 & 0 \\
\bar{e}_{5 L} & 0 & 0 & 0 & M_{54}^{\prime \prime e} & \tilde{M}_{55}^{e} & 0 & 0
\end{array}\right),
$$

where the indices $\alpha, \beta$ run from 1 to 5 , and tilde, primes repeated in the mass matrix mean that the parameters are rotated. The unitary $5 \times 5$ matrices are defined to be

$$
\begin{aligned}
& V_{L}=V_{45}^{L} V_{35}^{L} V_{25}^{L} V_{15}^{L} V_{34}^{L} V_{24}^{L} V_{14}^{L}, \\
& V_{e}=V_{45}^{e} V_{35}^{e} V_{25}^{e} V_{15}^{e} V_{34}^{e} V_{24}^{e} V_{14}^{e},
\end{aligned}
$$

where each of the unitary matrices $V_{i 4.5}$ are parameterized by a single angle $\theta_{i 4,5}$ describing the mixing between the $i$ th chiral family and the 4,5 th vectorlike family. The $5 \times 5$
Yukawa constant matrix in a mass basis (primed) can be diagonalized by the unitary rotation matrices as below:

$$
\tilde{y}_{\alpha \beta}^{e}=V_{L} \tilde{y}_{\alpha \beta}^{\prime e} V_{e}^{\dagger}
$$

From Eq. (7), we can read off the $5 \times 5$ upper block and confirm that the $(3,4),(1,5),(2,5),(3,5)$ mixings in the $L$ sector and $(2,4),(3,4),(1,5),(2,5),(3,5)$ mixings in the $e$ sector are required to go to the decoupling basis. The unitary matrices of Eq. (10) and mixing angles appearing in the unitary matrices are parameterized by 


$$
\begin{aligned}
& V_{L}=V_{35}^{L} V_{25}^{L} V_{15}^{L} V_{34}^{L} \\
& =\left(\begin{array}{ccccc}
1 & 0 & 0 & 0 & 0 \\
0 & 1 & 0 & 0 & 0 \\
0 & 0 & c_{35}^{L} & 0 & s_{35}^{L} \\
0 & 0 & 0 & 1 & 0 \\
0 & 0 & -s_{35}^{L} & 0 & c_{35}^{L}
\end{array}\right)\left(\begin{array}{ccccc}
1 & 0 & 0 & 0 & 0 \\
0 & c_{25}^{L} & 0 & 0 & s_{25}^{L} \\
0 & 0 & 1 & 0 & 0 \\
0 & 0 & 0 & 1 & 0 \\
0 & -s_{25}^{L} & 0 & 0 & c_{25}^{L}
\end{array}\right)\left(\begin{array}{ccccc}
c_{15}^{L} & 0 & 0 & 0 & s_{15}^{L} \\
0 & 1 & 0 & 0 & 0 \\
0 & 0 & 1 & 0 & 0 \\
0 & 0 & 0 & 1 & 0 \\
-s_{15}^{L} & 0 & 0 & 0 & c_{15}^{L}
\end{array}\right)\left(\begin{array}{ccccc}
1 & 0 & 0 & 0 & 0 \\
0 & 1 & 0 & 0 & 0 \\
0 & 0 & c_{34}^{L} & s_{34}^{L} & 0 \\
0 & 0 & -s_{34}^{L} & c_{34}^{L} & 0 \\
0 & 0 & 0 & 0 & 1
\end{array}\right) \\
& \approx\left(\begin{array}{ccccc}
1 & 0 & 0 & 0 & s_{15}^{L} \\
0 & 1 & 0 & 0 & s_{25}^{L} \\
0 & 0 & 1 & s_{34}^{L} & s_{35}^{L} \\
0 & 0 & -s_{34}^{L} & 1 & 0 \\
-s_{15}^{L} & -s_{25}^{L} & -s_{35}^{L} & 0 & 1
\end{array}\right) \\
& s_{34}^{L}=\frac{x_{34}^{L}\langle\phi\rangle}{\sqrt{\left(x_{34}^{L}\langle\phi\rangle\right)^{2}+\left(M_{44}^{L}\right)^{2}}}, \quad s_{15}^{L}=\frac{x_{15}^{L}\langle\phi\rangle}{\sqrt{\left(x_{15}^{L}\langle\phi\rangle\right)^{2}+\left(M_{55}^{L}\right)^{2}}}, \\
& s_{25}^{L}=\frac{x_{25}^{L}\langle\phi\rangle}{\sqrt{\left(x_{25}^{L}\langle\phi\rangle\right)^{2}+\left(M_{55}^{\prime L}\right)^{2}}}, \quad s_{35}^{L}=\frac{x_{35}^{\prime L}\langle\phi\rangle}{\sqrt{\left(x_{35}^{\prime L}\langle\phi\rangle\right)^{2}+\left(M_{55}^{\prime \prime L}\right)^{2}}}, \\
& x_{35}^{\prime L}\langle\phi\rangle=c_{34}^{L} x_{35}^{L}\langle\phi\rangle+s_{34}^{L} M_{45}^{L}, \quad M_{45}^{\prime L}=-s_{34}^{L} x_{35}^{L}\langle\phi\rangle+c_{34}^{L} M_{45}^{L} \quad \tilde{M}_{44}^{L}=\sqrt{\left(x_{34}^{L}\langle\phi\rangle\right)^{2}+\left(M_{44}^{L}\right)^{2}}, \\
& M_{55}^{\prime L}=\sqrt{\left(x_{15}^{L}\langle\phi\rangle\right)^{2}+\left(M_{55}^{L}\right)^{2}}, \quad M_{55}^{\prime \prime L}=\sqrt{\left(x_{25}^{L}\langle\phi\rangle\right)^{2}+\left(M_{55}^{\prime L}\right)^{2}}, \quad \tilde{M}_{55}^{L}=\sqrt{\left(x_{35}^{\prime L}\langle\phi\rangle\right)^{2}+\left(M_{55}^{\prime \prime L}\right)^{2}} \\
& V_{e}=V_{35}^{e} V_{25}^{e} V_{15}^{e} V_{34}^{e} V_{24}^{e} \\
& =\left(\begin{array}{ccccc}
1 & 0 & 0 & 0 & 0 \\
0 & 1 & 0 & 0 & 0 \\
0 & 0 & c_{35}^{e} & 0 & s_{35}^{e} \\
0 & 0 & 0 & 1 & 0 \\
0 & 0 & -s_{35}^{e} & 0 & c_{35}^{e}
\end{array}\right)\left(\begin{array}{ccccc}
1 & 0 & 0 & 0 & 0 \\
0 & c_{25}^{e} & 0 & 0 & s_{25}^{e} \\
0 & 0 & 1 & 0 & 0 \\
0 & 0 & 0 & 1 & 0 \\
0 & -s_{25}^{e} & 0 & 0 & c_{25}^{e}
\end{array}\right)\left(\begin{array}{ccccc}
c_{15}^{e} & 0 & 0 & 0 & s_{15}^{e} \\
0 & 1 & 0 & 0 & 0 \\
0 & 0 & 1 & 0 & 0 \\
0 & 0 & 0 & 1 & 0 \\
-s_{15}^{e} & 0 & 0 & 0 & c_{15}^{e}
\end{array}\right) \\
& \times\left(\begin{array}{ccccc}
1 & 0 & 0 & 0 & 0 \\
0 & 1 & 0 & 0 & 0 \\
0 & 0 & c_{34}^{e} & s_{34}^{e} & 0 \\
0 & 0 & -s_{34}^{e} & c_{34}^{e} & 0 \\
0 & 0 & 0 & 0 & 1
\end{array}\right)\left(\begin{array}{ccccc}
1 & 0 & 0 & 0 & 0 \\
0 & c_{24}^{e} & 0 & s_{24}^{e} & 0 \\
0 & 0 & 1 & 0 & 0 \\
0 & -s_{24}^{e} & 0 & c_{24}^{e} & 0 \\
0 & 0 & 0 & 0 & 1
\end{array}\right) \approx\left(\begin{array}{ccccc}
1 & 0 & 0 & 0 & \theta_{15}^{e} \\
0 & 1 & 0 & \theta_{24}^{e} & \theta_{25}^{e} \\
0 & 0 & 1 & \theta_{34}^{e} & \theta_{35}^{e} \\
0 & -\theta_{24}^{e} & -\theta_{34}^{e} & 1 & 0 \\
-\theta_{15}^{e} & -\theta_{25}^{e} & -\theta_{35}^{e} & 0 & 1
\end{array}\right), \\
& s_{24}^{e} \approx \frac{x_{42}^{e}\langle\phi\rangle}{M_{44}^{e}}, \quad s_{34}^{e} \approx \frac{x_{43}^{e}\langle\phi\rangle}{M_{44}^{\prime e}}, \quad s_{15}^{e} \approx \frac{x_{51}^{e}\langle\phi\rangle}{M_{55}^{e}}, \quad s_{25}^{e} \approx \frac{x_{52}^{\prime e}\langle\phi\rangle}{M_{55}^{\prime e}}, \quad s_{35}^{e} \approx \frac{x_{53}^{e}\langle\phi\rangle}{M_{55}^{\prime \prime e}}, \\
& x_{52}^{\prime e}\langle\phi\rangle=c_{24}^{e} x_{52}^{e}\langle\phi\rangle+s_{24}^{e} M_{54}^{e}, \quad M_{54}^{\prime e}=-s_{24}^{e} x_{52}^{e}\langle\phi\rangle+c_{24}^{e} M_{54}^{e}, \\
& x_{53}^{\prime e}\langle\phi\rangle=c_{34}^{e} x_{53}^{e}\langle\phi\rangle+s_{34}^{e} M_{54}^{\prime e}, \quad M_{54}^{\prime \prime e}=-s_{34}^{e} x_{53}^{e}\langle\phi\rangle+c_{34}^{e} M_{54}^{\prime e}, \\
& M_{44}^{\prime e}=\sqrt{\left(x_{42}^{e}\langle\phi\rangle\right)^{2}+\left(M_{44}^{e}\right)^{2}}, \quad \tilde{M}_{44}^{e}=\sqrt{\left(x_{43}^{e}\langle\phi\rangle\right)^{2}+\left(M_{44}^{e}\right)^{2}}, \\
& M_{55}^{\prime e}=\sqrt{\left(x_{51}^{e}\langle\phi\rangle\right)^{2}+\left(M_{55}^{e}\right)^{2}}, \quad M_{55}^{\prime \prime e}=\sqrt{\left(x_{52}^{\prime e}\langle\phi\rangle\right)^{2}+\left(M_{55}^{\prime e}\right)^{2}}, \quad \tilde{M}_{55}^{e}=\sqrt{\left(x_{53}^{\prime e}\langle\phi\rangle\right)^{2}+\left(M_{55}^{\prime \prime e}\right)^{2}} .
\end{aligned}
$$


Given the above unitary rotations, the $5 \times 5$ Yukawa matrices are computed in terms of the mixing angles and the upper $3 \times 3$ block would be the effective SM Yukawa matrix. Assuming all $\cos \theta$ to be 1 and neglecting order of $\theta$ square or more than that, we have a simple $3 \times 3$ Yukawa matrix of Eq. (14).

$$
y_{i j}^{e}=\left(\begin{array}{ccc}
s_{15}^{L} y_{51}^{e}+y_{15}^{e} \theta_{15}^{e} & s_{15}^{L} y_{52}^{e}+y_{15}^{e} \theta_{25}^{e} & s_{15}^{L} y_{53}^{e}+y_{15}^{e} \theta_{35}^{e} \\
s_{25}^{L} y_{51}^{e}+y_{25}^{e} \theta_{15}^{e} & s_{25}^{L} y_{52}^{e}+y_{24}^{e} \theta_{24}^{e}+y_{25}^{e} \theta_{25}^{e} & s_{25}^{L} y_{53}^{e}+y_{24}^{e} \theta_{34}^{e}+y_{25}^{e} \theta_{35}^{e} \\
s_{35}^{L} y_{51}^{e}+y_{35}^{e} \theta_{15}^{e} & s_{35}^{L} y_{52}^{e}+y_{34}^{e} \theta_{24}^{e}+y_{35}^{e} \theta_{25}^{e} & s_{34}^{L} y_{43}^{e}+s_{35}^{L} y_{53}^{e}+y_{34}^{e} \theta_{34}^{e}+y_{35}^{e} \theta_{35}^{e}
\end{array}\right)
$$

\section{A convenient basis for neutrinos}

The relevant Yukawa and mass terms of the neutrino sector give rise to the following neutrino mass matrix:

$$
M^{\nu}=\left(\begin{array}{c|ccc|cccc} 
& L_{1 L} & L_{2 L} & L_{3 L} & \bar{\nu}_{4 R} & \bar{\nu}_{5 R} & \tilde{\nu}_{4 R} & \tilde{\nu}_{5 R} \\
\hline L_{1 L} & 0 & 0 & 0 & y_{14}^{\nu} v_{u} & y_{15}^{\nu} v_{u} & x_{14}^{L} v_{d} & x_{15}^{L} v_{d} \\
L_{2 L} & 0 & 0 & 0 & y_{24}^{\nu} v_{u} & y_{25}^{\nu} v_{u} & x_{24}^{L} v_{d} & x_{25}^{L} v_{d} \\
L_{3 L} & 0 & 0 & 0 & y_{34}^{\nu} v_{u} & y_{35}^{\nu} v_{u} & x_{34}^{L} v_{d} & x_{35}^{L} v_{d} \\
\hline \bar{\nu}_{4 R} & y_{14}^{\nu} v_{u} & y_{24}^{\nu} v_{u} & y_{34}^{\nu} v_{u} & 0 & 0 & M_{44}^{\nu} & M_{54}^{\nu} \\
\bar{\nu}_{5 R} & y_{15}^{\nu} v_{u} & y_{25}^{\nu} v_{u} & y_{35}^{\nu} v_{u} & 0 & 0 & M_{45}^{\nu} & M_{55}^{\nu} \\
\tilde{\nu}_{4 R} & x_{14}^{L} v_{d} & x_{24}^{L} v_{d} & x_{34}^{L} v_{d} & M_{44}^{\nu} & M_{45}^{\nu} & 0 & 0 \\
\tilde{\nu}_{5 R} & x_{15}^{L} v_{d} & x_{25}^{L} v_{d} & x_{35}^{L} v_{d} & M_{54}^{\nu} & M_{55}^{\nu} & 0 & 0
\end{array}\right)
$$

Here, the zeros in the upper $3 \times 3$ block of Eq. (15) mean that neutrinos remain massless in the SM. Therefore, the SM neutrinos can be massive via the inclusion of two vectorlike families. In order to make this mass matrix as simple as possible, the only choice left is to rotate $\nu_{4 R}$ and $\nu_{5 R}$ to turn off $M_{45}^{\nu}$ since rotations between $L_{1 L, 2 L, 3 L}$ are already used in the charged lepton sector.

\section{IV. $W$ BOSON EXCHANGE CONTRIBUTIONS TO $(g-2)_{\mu},(g-2)_{e}$, AND BR $(\mu \rightarrow e \gamma)$}

Within the framework of our proposed model, we start by investigating the muon and electron anomalous magnetic moments with $W$ boson exchange first. Given that such $W$ boson exchange contribution also involves virtual neutrinos in the internal lines of the loop, we revisit the mass matrix for neutrinos. In this mass matrix, we remove fifth vectorlike neutrinos $\nu_{5 R}$ and $\tilde{\nu}_{5 R}$ since they are too heavy to contribute to the phenomenology under study. As mentioned in the previous section, we stick to a condition where the coefficient $y$ is expected to be of order unity, whereas the coupling $x$ is expected to be smaller than $y$. Such condition can be easily seen by substituting the coefficients $y_{i 4}^{\nu}$ by $y_{i}^{\nu}$ and the coefficients $x_{i 4}^{L}$ by $\epsilon y_{i}^{\nu \prime}$ where $\epsilon$ is a suppression factor. Putting all these considerations together, the mass matrix for neutrinos in Eq. (15) after electroweak symmetry breaking takes the form:

$$
M^{\nu} \approx\left(\begin{array}{c|ccc|cc} 
& \nu_{1 L} & \nu_{2 L} & \nu_{3 L} & \bar{\nu}_{4 R} & \tilde{\nu}_{4 R} \\
\hline \nu_{1 L} & 0 & 0 & 0 & y_{1}^{\nu} v_{u} & \epsilon y_{1}^{\nu \prime} v_{d} \\
\nu_{2 L} & 0 & 0 & 0 & y_{2}^{\nu} v_{u} & \epsilon y_{2}^{\nu \prime} v_{d} \\
\nu_{3 L} & 0 & 0 & 0 & y_{3}^{\nu} v_{u} & \epsilon y_{3}^{\nu \prime} v_{d} \\
\hline \bar{\nu}_{4 R} & y_{1}^{\nu} v_{u} & y_{2}^{\nu} v_{u} & y_{3}^{\nu} v_{u} & 0 & M_{44}^{\nu} \\
\tilde{\nu}_{4 R} & \epsilon y_{1}^{\nu \prime} v_{d} & \epsilon y_{2}^{\nu \prime} v_{d} & \epsilon y_{3}^{\nu \prime} v_{d} & M_{44}^{\nu} & 0
\end{array}\right) \equiv\left(\begin{array}{cc}
0 & m_{D} \\
m_{D}^{T} & M_{N}
\end{array}\right),
$$

where $v_{u}\left(v_{d}\right)$ is the vev of $\tilde{H}_{u}\left(H_{d}\right), v_{u}$ runs from $246 / \sqrt{2} \mathrm{GeV} \simeq 174 \mathrm{GeV}$ to $246 \mathrm{GeV}$ and $v_{u}^{2}+v_{d}^{2}=(246 \mathrm{GeV})^{2}$. 


\section{A. Type 1b seesaw mechanism}

Now that we constructed the neutrino mass matrix for this task, the next step is to read off the operator which gives rise to the neutrino mass from the mass matrix. Generally, the well-known operator for neutrino mass is the Weinberg operator (type 1a seesaw mechanism) $\frac{1}{\Lambda} L_{i} L_{j} H H$. A main feature of the Weinberg operator is the same SM Higgs should be repeated in the operator, however that property is not present in our model since the Higgs doublets $H_{u, d}$ are negatively charged under the $U(1)^{\prime}$ symmetry, which implies the corresponding Weinberg operator having such fields will not be invariant under the $U(1)^{\prime}$ unless an insertion of a quadratic power of the gauge singlet scalar $\phi$ is considered. However we do not consider the operators $\frac{1}{\Lambda^{3}}\left(\bar{L}_{i} \tilde{H}_{u}\right)\left(\tilde{H}_{u} L_{j}^{C}\right)\left(\phi^{*}\right)^{2}$ and $\frac{1}{\Lambda^{3}}\left(\bar{L}_{i} H_{d}\right)\left(H_{d} L_{j}^{C}\right) \phi^{2}$ in the neutrino sector, since they are very subleading and thus will give a tiny contribution to the light active neutrino masses. Instead of relying on a seven dimensional Weinberg to generate the tiny masses for the light active neutrinos, we take another approach named type 1b seesaw mechanism (we call the Weinberg operator "type 1a seesaw mechanism" to differentiate with) where the mixing of different $S U(2)$ Higgs doublets can appear satisfying charge conservation. Diagrams for the operators are given in Fig. 5 for comparison:

The diagrams in Fig. 5 clearly tell the difference between Majorana mass and vectorlike mass. They share a common property that they violate the lepton number conservation, whereas the particles appearing in a Majorana mass term are same but those ones involved in vectorlike mass terms are different. As the type $1 \mathrm{~b}$ seesaw mechanism only works in this model, we make use of this seesaw mechanism for the analysis of neutrinos. With the operator, the renormalizable Lagrangian for neutrinos can be written as:

$$
\begin{aligned}
\mathcal{L}_{\nu}^{\text {Yukawa+Mass }}= & y_{i}^{\nu} \bar{L}_{i L} \tilde{H}_{u} \nu_{k R}+\epsilon y_{i}^{\nu \prime} \bar{L}_{i L} H_{d} \overline{\tilde{\nu}}_{k R}+M_{k k}^{M} \overline{\tilde{\nu}}_{k R} \nu_{k R} \\
& + \text { H.c., }
\end{aligned}
$$

where $i=1,2,3$ and $k=4$. The renormalizable Lagrangian of Eq. (17) above the electroweak scale generates an effective Lagrangian after decoupling the heavy vectorlike neutrinos, which is suitable for study of low energy neutrino phenomenology. The effective Lagrangian for neutrino at electroweak scale is given by [11].

$\mathcal{L}^{d=5}=c_{i j}^{d=5}\left(\left(L_{i}^{T} \tilde{H}_{u}\right)\left(H_{d}^{T} L_{j}\right)+\left(L_{i}^{T} H_{d}\right)\left(\tilde{H}_{u}^{T} L_{j}\right)\right)$,

where the coefficient $c_{i j}^{d=5}$ is suppressed by a factor of the vectorlike mass $M$. The neutrino mass matrix of Eq. (16) can be diagonalized by the unitary matrix $U$ as below:

$$
U^{T}\left(\begin{array}{cc}
0 & m_{D}^{T} \\
m_{D} & M_{N}
\end{array}\right) U=\left(\begin{array}{cc}
m_{\nu}^{\mathrm{diag}} & 0 \\
0 & M_{N}^{\mathrm{diag}}
\end{array}\right),
$$

where $m_{\nu}^{\text {diag }}$ is a diagonal matrix for the light left-handed neutrinos $\nu_{i L}$ and $M_{N}^{\text {diag }}$ is that for the heavy vectorlike neutrinos $\nu_{4 R}, \tilde{\nu}_{4 R}$. Here, the unitary mixing matrix $U$ is defined by multiplication of two unitary matrices which we call $U_{A}$ and $U_{B}$, respectively [78]:

$$
\begin{aligned}
U & =U_{A} \cdot U_{B} \\
U_{A} & =\exp \left(\begin{array}{cc}
0 & \Theta \\
-\Theta^{\dagger} & 0
\end{array}\right) \simeq\left(\begin{array}{cc}
I-\frac{\Theta \Theta^{\dagger}}{2} & \Theta \\
-\Theta^{\dagger} & I-\frac{\Theta \Theta^{\dagger}}{2}
\end{array}\right) \text { at leading order in } \Theta \\
U_{B} & =\left(\begin{array}{cc}
U_{\mathrm{PMNS}} & 0 \\
0 & I
\end{array}\right)
\end{aligned}
$$

The unitary matrix $U_{\mathrm{PMNS}}$ in $U_{B}$ is the well-known Pontecorvo-Maki-Nakagawa-Sakata matrix and is parametrized by $[11,79]$.
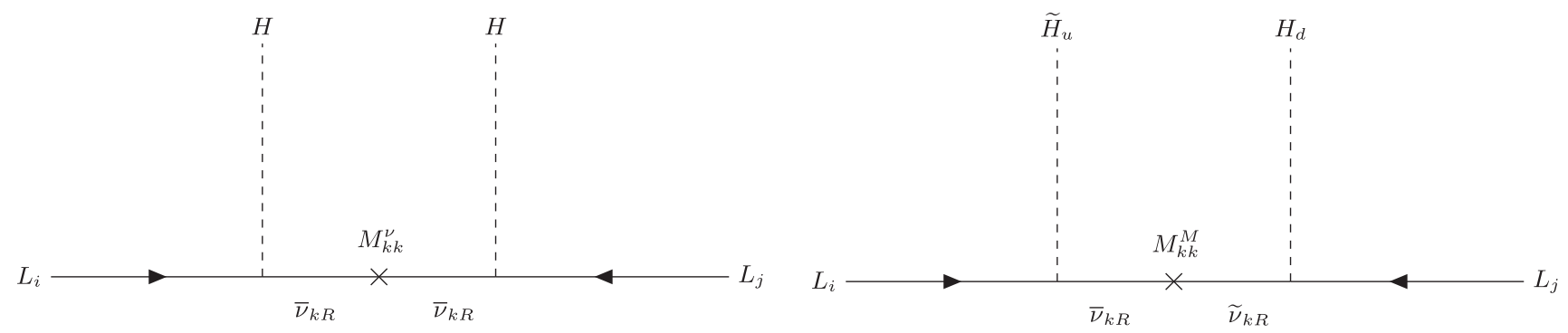

FIG. 5. Diagrams which lead to effective Weinberg operators for the Majorana and vectorlike mass in the mass insertion formalism, where $i, j=1,2,3$ and $k=4$, respectively. The left is the Weinberg operator(or type 1a seesaw mechanism) in which mass $M$ is Majorana mass and the right is Weinberg-like operator(or type 1b seesaw mechanism) in which mass $M$ is vectorlike mass. 


$$
U_{\mathrm{PMNS}}=\left(\begin{array}{ccc}
1 & 0 & 0 \\
0 & \cos \theta_{23} & \sin \theta_{23} \\
0 & -\sin \theta_{23} & \cos \theta_{23}
\end{array}\right)\left(\begin{array}{ccc}
\cos \theta_{13} & 0 & \sin \theta_{13} e^{-i \delta_{C P}} \\
0 & 1 & 0 \\
-\sin \theta_{13} e^{i \delta_{C P}} & 0 & \cos \theta_{13}
\end{array}\right)\left(\begin{array}{ccc}
\cos \theta_{12} & \sin \theta_{12} & 0 \\
-\sin \theta_{12} & \cos \theta_{12} & 0 \\
0 & 0 & 1
\end{array}\right)\left(\begin{array}{ccc}
e^{-i \alpha^{\prime} / 2} & 0 & 0 \\
0 & e^{-i \alpha / 2} & 0 \\
0 & 0 & 1
\end{array}\right),
$$

where the Majorana phase $\alpha^{\prime}$ is set to zero in this model. The mixing matrices $U_{A, B}$ are unitary, however the $3 \times 3$ upper block of the unitary matrix $U$ is not unitary due to the factor $\left(I-\Theta \Theta^{\dagger} / 2\right)$ for the light neutrinos. An interesting feature of the unitary matrix $U$ is it is unitary globally, but nonunitary locally and this nonunitarity contributes to explain muon and electron anomalous magnetic moments. Replacing the unitary matrices in Eq. (20) back to Eq. (19), the result is simplified with the assumption $M_{N} \gg m_{D}$ to the conventional seesaw mechanism:

$$
\begin{aligned}
\Theta & \simeq m_{D}^{\dagger} M_{N}^{-1} \\
U_{\mathrm{PMNS}}^{*} m_{\nu}^{\text {diag }} U_{\mathrm{PMNS}}^{\dagger} & \simeq-m_{D}^{T} M_{N}^{-1} m_{D} \equiv-m \\
M_{N}^{\text {diag }} & \simeq M_{N},
\end{aligned}
$$

where $m$ is the effective mass matrix resulted from Eq. (16).

$$
m_{i j}=\frac{\epsilon v_{u} v_{d}}{M_{44}^{\nu}}\left(y_{i}^{\nu} y_{j}^{\nu \prime}+y_{i}^{\nu \prime} y_{j}^{\nu}\right)
$$

Therefore, smallness of the light neutrino masses can be understood not only from mass of vectorlike mass $M_{44}^{\nu}$ but also from the suppression factor $\epsilon$ and the presence of $\epsilon$ allows more flexibility in the allowed mass values of the vectorlike neutrinos. Revisiting nonunitarity part for the light neutrinos from the unitary matrix $U[78,80]$, it reads:

$$
\left(I-\frac{\Theta_{i} \Theta_{j}^{\dagger}}{2}\right) U_{\mathrm{PMNS}}=\left(I-\eta_{i j}\right) U_{\mathrm{PMNS}}
$$

The nonunitarity $\eta$ is associated with the presence of the heavy vectorlike neutrinos and can be derived from a coefficient of the effective Lagrangian at dimension 6 [81]:

$$
\mathcal{L}^{d=6}=c_{i j}^{d=6}\left(\left(L_{i}^{\dagger} \tilde{H}_{u}\right) i \not \partial\left(\tilde{H}_{u}^{\dagger} L_{j}\right)+\left(L_{i}^{\dagger} H_{d}\right) i \not \partial\left(H_{d}^{\dagger} L_{j}\right)\right)
$$

Once the SM Higgs doublets in Eq. (25) develop its vev, the Lagrangian at dimension 6 causes nondiagonal kinetic terms for the light neutrinos and it gives rise to deviations of unitarity when it is diagonalized. The deviations of unitarity can be expressed in terms of the coefficient at dimension 6 $\eta_{i j} \equiv v^{2} c_{i j}^{d=6} / 2$.

$$
\begin{aligned}
\eta_{i j} & =\frac{\Theta_{i} \Theta_{j}^{\dagger}}{2}=\frac{1}{2} \frac{m_{D}^{\dagger} m_{D}}{M_{N}^{2}} \\
& =\frac{1}{2 M_{44}^{\nu 2}}\left(v_{u}^{2} y_{i}^{\nu *} y_{j}^{\nu}+\epsilon^{2} v_{d}^{2} y_{i}^{\nu / *} y_{j}^{\nu \prime}\right) \simeq \frac{v_{u}^{2}}{2 M_{44}^{\nu 2}} y_{i}^{\nu *} y_{j}^{\nu}
\end{aligned}
$$

From the fourth term in Eq. (26), the term with $\epsilon^{2}$ can be safely ignored due to both relative smallness of $v_{d}$ and the suppression factor $\epsilon$. Thus, the deviation of unitarity $\eta$ consists of the vectorlike mass $M_{44}^{\nu}$ and the Yukawa couplings $y_{i, j}^{\nu}$. As an interesting example, it is possible that the Yukawa couplings $y_{i, j}^{\nu}$ can be obtained from the observables such as the PMNS mixing matrix and two mass squared splitting, $\Delta m_{\text {sol }}^{2}$ and $\Delta m_{\text {atm }}^{2}$, in the neutrino oscillation experiments. Since the hierarchy between the light neutrinos is not yet determined, there are two possible scenarios, normal hierarchy( $\mathrm{NH})$ and inverted hierarchy (IH), and the lightest neutrino remains massless, whereas two other neutrinos get massive. The Yukawa couplings $y_{i}^{\nu, \nu \prime}$ for the $\mathrm{NH}\left(m_{1}=0\right)$ are determined by

$$
\begin{aligned}
& y_{i}^{\nu}=\frac{y}{\sqrt{2}}\left(\sqrt{1+\rho}\left(U_{\mathrm{PMNS}}^{*}\right)_{i 3}+\sqrt{1-\rho}\left(U_{\mathrm{PMNS}}^{*}\right)_{i 2}\right) \\
& y_{i}^{\nu^{\prime \prime}}=\frac{y^{\prime}}{\sqrt{2}}\left(\sqrt{1+\rho}\left(U_{\mathrm{PMNS}}^{*}\right)_{i 3}-\sqrt{1-\rho}\left(U_{\mathrm{PMNS}}^{*}\right)_{i 2}\right),
\end{aligned}
$$

where $y$ and $y^{\prime}$ are real numbers and $\rho=(1-\sqrt{r}) /(1+\sqrt{r})$ with $r \equiv\left|\Delta m_{\text {sol }}^{2}\right| /\left|\Delta m_{\text {atm }}^{2}\right|=\Delta m_{21}^{2} / \Delta m_{31}^{2}$, whereas the Yukawa couplings $y_{i}^{\nu, \nu \prime}$ for the $\operatorname{IN}\left(m_{3}=0\right)$ are

$$
\begin{aligned}
y_{i}^{\nu} & =\frac{y}{\sqrt{2}}\left(\sqrt{1+\rho}\left(U_{\mathrm{PMNS}}^{*}\right)_{i 2}+\sqrt{1-\rho}\left(U_{\mathrm{PMNS}}^{*}\right)_{i 1}\right) \\
y_{i}^{\nu \prime} & =\frac{y^{\prime}}{\sqrt{2}}\left(\sqrt{1+\rho}\left(U_{\mathrm{PMNS}}^{*}\right)_{i 2}-\sqrt{1-\rho}\left(U_{\mathrm{PMNS}}^{*}\right)_{i 1}\right),
\end{aligned}
$$

where $\rho=(1-\sqrt{1+r}) /(1+\sqrt{1+r}) \quad$ with $\quad r \equiv$ $\left|\Delta m_{\mathrm{sol}}^{2}\right| /\left|\Delta m_{\mathrm{atm}}^{2}\right|=\Delta m_{21}^{2} / \Delta m_{32}^{2}$.

\section{B. The charged lepton flavor violation(CLFV) $\mu \rightarrow e \gamma$ decay}

Consider the three light neutrinos in the SM for the CLFV $\mu \rightarrow e \gamma$ decay first. In this case, the unitary mixing matrix becomes just the PMNS mixing matrix and the GIM mechanism which suppresses flavor-changing process works, therefore it leads quite suppressed sensitivity for $\operatorname{BR}(\mu \rightarrow e \gamma)$ about $10^{-55}$ [82], which is impossible to 

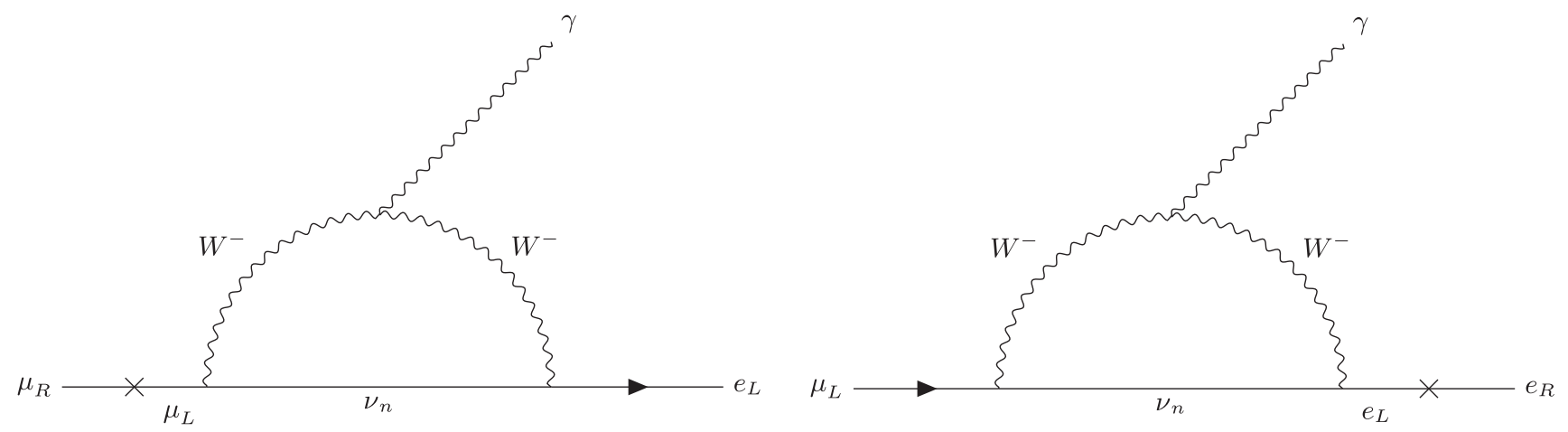

FIG. 6. Diagrams for CLFV $\mu \rightarrow e \gamma$ decay with all neutrinos. Here $n=1,2,3,4,5$.

observe with the current sensitivity of $\mu \rightarrow e \gamma$ decay. This impractical sensitivity can be enhanced to the observable level by introducing the heavy vectorlike neutrinos which give rise to deviation of unitarity. With the presence of heavy vectorlike neutrinos, the GIM mechanism is gone and the factor suppressed by GIM mechanism can survive with a factor of deviation of unitarity, which plays a crucial role to increase significantly order of theoretical prediction for $\mu \rightarrow e \gamma$ decay [83]. Therefore, the strongest constraint for deviation of unitarity in the modified PMNS mixing matrix comes from CLFV $\mu \rightarrow e \gamma$ decay. The possible oneloop diagrams for the CLFV $\mu \rightarrow e \gamma$ with all neutrinos in this model are given in Figure 6.

The amplitude from above diagrams in Figure 6 reads [82]:

$$
\begin{aligned}
\mathcal{M}(\mu \rightarrow e \gamma) & =\bar{u}_{e} i \sigma_{\mu \nu} q^{\nu}\left(F_{1}+F_{2} \gamma^{5}\right) u_{\mu} \epsilon^{* \mu} \\
& =\bar{u}_{e} i \sigma_{\mu \nu} q^{\nu}\left(A_{R} P_{R}+A_{L} P_{L}\right) u_{\mu} \epsilon^{* \mu},
\end{aligned}
$$

where $u$ is Dirac spinor for the muon and electron, $q$ is four momentum of an outgoing photon, $F_{1,2}$ are form factors, $A_{L, R}$ are left- and right-handed amplitude defined to be $A_{L, R}=F_{1} \pm F_{2}$ and lastly $P_{L, R}$ are projection operators. From the amplitude, the helicity flip between initial particle and final particle should arise and this makes the helicity flip process takes place on one of external legs since the $W$ gauge boson couples only to left-handed fields. Comparing the left diagram with the right, the left is proportional to the muon mass, while the right is proportional to the electron mass, which means that impact of the right is ignorable. The unpolarized squared amplitude $|\mathcal{M}|^{2}$ takes the form:

$$
|\mathcal{M}|^{2}=m_{\mu}^{4}\left(A_{R}+A_{L}\right)^{2} \simeq m_{\mu}^{4}\left(A_{R}\right)^{2}
$$

Then, the decay rate is given by

$$
\Gamma(\mu \rightarrow e \gamma)=\frac{|\mathcal{M}|^{2}}{16 \pi m_{\mu}}=\frac{m_{\mu}^{3}}{16 \pi}\left|A_{R}\right|^{2}
$$

where $A_{R}$ is expressed by $[82,84]^{3}$

$$
\begin{aligned}
A_{R}= & \frac{g^{2} e}{128 \pi^{2}} \frac{m_{\mu}}{M_{W}^{2}} \sum_{n=1,2,3,4,5} U_{2 n} U_{1 n}^{*} F\left(x_{n}\right)\left[1-\frac{1}{3} \frac{\ln \xi}{\xi-1}\right. \\
& \left.+\frac{1}{\xi-1}\left(\frac{\xi \ln \xi}{\xi-1}-1\right)\right]
\end{aligned}
$$

Taking the unitary gauge into account, $\xi \rightarrow \infty$, the additional $\xi$-dependent terms in $A_{R}$ all are cancelled by contribution of Goldstone bosons so $A_{R}$ is gauge invariant. Substituting the gauge invariant $A_{R}$ back into the decay rate of Eq. (31) and dividing the expanded decay rate by the total muon decay rate $\Gamma(\mu \rightarrow e \nu \bar{\nu})=G_{F}^{2} m_{\mu}^{5} / 192 \pi^{3}$, we have the prediction for $\mu \rightarrow e \gamma$ decay $[11,82]$ :

$$
\begin{aligned}
\operatorname{BR}(\mu \rightarrow e \gamma) & =\frac{\Gamma(\mu \rightarrow e \gamma)}{\Gamma\left(\mu \rightarrow e \nu_{\mu} \bar{\nu}_{e}\right)} \\
& =\frac{3 \alpha}{32 \pi} \frac{\left|\sum_{n=1}^{5} U_{2 n} U_{n 1}^{\dagger} F\left(x_{n}\right)\right|^{2}}{\left(U U^{\dagger}\right)_{11}\left(U U^{\dagger}\right)_{22}},
\end{aligned}
$$

where $x_{n}=M_{n}^{2} / M_{W}^{2}$ and the loop function $F\left(x_{n}\right)$ is

$F\left(x_{n}\right)=\frac{10-43 x_{n}+78 x_{n}^{2}-\left(49-18 \log x_{n}\right) x_{n}^{3}+4 x_{n}^{4}}{3\left(x_{n}-1\right)^{4}}$.

Numerator in Eq. (33) can be simplified by separating the light neutrinos and heavy vectorlike neutrinos as below (Contribution of the fifth neutrino $\tilde{\nu}_{4 R}$ is safely ignored both by the suppression factor $\epsilon$ and by relative smallness of $v_{d}$ compared to $v_{u}$ ):

\footnotetext{
${ }^{3}$ Since the PMNS mixing matrix is multiplied by a factor of deviation of unitarity, it is not unitary anymore. Therefore, the first term of sum over neutrino eigenstates in Eq. (28) of [82] does not vanish and come in our prediction with a loop function $F\left(x_{n}\right)$.
} 

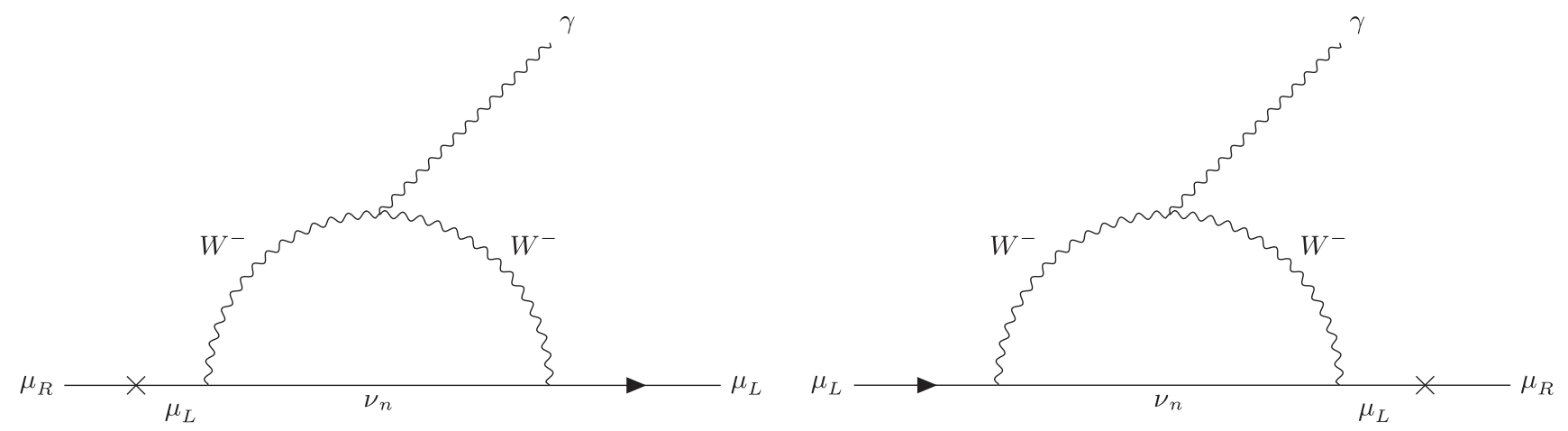

FIG. 7. Diagrams for muon anomalous magnetic moment with all neutrinos. Here $n=1,2,3,4,5$.

$$
\begin{aligned}
\left|\sum_{n=1}^{5} U_{2 n} U_{n 1}^{\dagger} F\left(x_{n}\right)\right|^{2} & \simeq\left|U_{2 i} U_{i 1}^{\dagger} F(0)+U_{24} U_{41}^{\dagger} F\left(x_{4}\right)\right|^{2} \\
U_{2 i} U_{i 1}^{\dagger} & =-\eta_{12}^{*}-\eta_{21}=-2 \eta_{21} \\
U_{24} U_{41}^{\dagger} & =\Theta_{24} \Theta_{14}^{*}=2 \eta_{21} \\
\left|\sum_{n=1}^{5} U_{2 n} U_{n 1}^{\dagger} F\left(x_{n}\right)\right|^{2} & \simeq\left|4 \eta_{21}\right|^{2}\left(F\left(x_{4}\right)-F\left(x_{0}\right)\right)^{2}
\end{aligned}
$$

The final form for the CLFV $\mu \rightarrow e \gamma$ decay in this model reads:

$$
\mathrm{BR}(\mu \rightarrow e \gamma)=\frac{3 \alpha_{e m}}{8 \pi}\left|\eta_{21}\right|^{2}\left(F\left(x_{4}\right)-F(0)\right)^{2},
$$

where $\alpha_{e m}$ is the fine structure constant. We find that our theoretical prediction for the $\mu \rightarrow e \gamma$ decay can be expressed in terms of the deviation of unitarity $\eta_{21}$.

\section{The anomalous muon magnetic moment $g-2$}

We derive our prediction for the muon anomalous magnetic moment in this section and confirm the derived expression can be consistent with an expression of the theoretical prediction for $\mu \rightarrow e \gamma$ in Refs. [82,85]. Consider two possible diagrams for muon anomalous magnetic moment at one-loop level in Fig. 7.

The amplitude for the muon anomalous magnetic moment at one-loop level is

$$
\begin{aligned}
\mathcal{M}\left(\Delta a_{\mu}\right) & =\bar{u}_{\mu} i \sigma_{\mu \nu} q^{\nu}\left(F_{1}+F_{2} \gamma^{5}\right) u_{\mu} \epsilon^{* \mu} \\
& =\bar{u}_{\mu} i \sigma_{\mu \nu} q^{\nu}\left(A_{R} P_{R}+A_{L} P_{L}\right) u_{\mu} \epsilon^{* \mu} .
\end{aligned}
$$

Unlike the CLFV $\mu \rightarrow e \gamma$ decay, muon anomaly diagrams have the same structure for helicity flip process. So we conclude $A_{R}$ is equal to $A_{L}$ and can make use of other expression of this amplitude to derive our own expression for $\Delta a_{\mu}$ [85].

$$
\begin{aligned}
V & =\bar{u}_{\mu} i \sigma_{\alpha \beta} q^{\beta} e m_{\mu}\left(A_{\mu \mu}^{M}+\gamma_{5} A_{\mu \mu}^{E}\right) u_{\mu} \epsilon^{* \alpha} \\
& =\bar{u}_{\mu} i \sigma_{\alpha \beta} q^{\beta} e m_{\mu}\left(\left(A_{\mu \mu}^{M}+A_{\mu \mu}^{E}\right) P_{R}+\left(A_{\mu \mu}^{M}-A_{\mu \mu}^{E}\right) P_{L}\right) u_{\mu} \epsilon^{* \alpha} .
\end{aligned}
$$

Comparing Eq. (37) with Eq. (38), we confirm that

$$
\begin{aligned}
& A_{R}=e m_{\mu}\left(A_{\mu \mu}^{M}+A_{\mu \mu}^{E}\right) \\
& A_{L}=e m_{\mu}\left(A_{\mu \mu}^{M}-A_{\mu \mu}^{E}\right) .
\end{aligned}
$$

Here, we can use the condition that $A_{R}=A_{L}$ identified in Fig. 7 and can rearrange $A_{L, R}$ in terms of $A_{\mu \mu}^{M, E}$, which are essential to derive our theoretical muon anomaly prediction. Then, we find our desirable form $A_{\mu \mu}^{M, E}$ for the muon anomalous magnetic moment.

$A_{\mu \mu}^{M}=\frac{1}{e m_{\mu}} A_{R}=\frac{g^{2}}{128 \pi^{2}} \frac{1}{M_{W}^{2}} \sum_{n=1,2,3,4,5} U_{2 n} U_{2 n}^{*} F\left(x_{n}\right)$

$A_{\mu \mu}^{E}=0$.

Using the definition for both the muon anomalous magnetic moment and branching ratio of $\mu \rightarrow e \gamma$ decay in [85], we can check our analytic argument for the observable and constraint are correct.

$$
\begin{aligned}
\Delta a_{\mu} & =A_{\mu \mu}^{M} m_{\mu}^{2} \\
\operatorname{BR}(\mu \rightarrow e \gamma) & =\frac{3(4 \pi)^{3} \alpha_{e m}}{4 G_{F}^{2}}\left(\left|A_{\mu e}^{M}\right|^{2}+\left|A_{\mu e}^{E}\right|^{2}\right) .
\end{aligned}
$$

One difference between $A_{\mu \mu}^{M, E}$ and $A_{L, R}$ is that $A_{\mu \mu}^{M, E}$ is only determined by the internal structure of the loop in Figure 7, whereas $A_{L, R}$ is the extended factor by multiplying $A_{\mu \mu}^{M, E}$ by the helicity flip mass in one of the external legs. Therefore, it is natural to think $A_{\mu \mu}^{M, E}$ is the same as $A_{\mu e}^{M, E}$ since their internal structure of loop are exactly same. ${ }^{4}$ The muon anomalous magnetic moment and the branching ratio of $\mu \rightarrow e \gamma$ take the form:

\footnotetext{
${ }^{4}$ One can concern the coefficient at the vertex with electron. However, this change is already reflected on the loop integration $A_{R}$ of Eq. (32) by $U_{1 n}$. For the muon anomaly, the coefficient is simply replaced by $U_{2 n}$, therefore, modification of the coefficient at the vertex does not harm our argument.
} 


$$
\begin{array}{r}
\Delta a_{\mu}=\frac{\alpha_{W}}{32 \pi} \frac{m_{\mu}^{2}}{M_{W}^{2}} \sum_{n=1,2,3,4,5} U_{2 n} U_{2 n}^{*} F\left(x_{n}\right) \\
\operatorname{BR}(\mu \rightarrow e \gamma)=\frac{3 \alpha_{e m}}{32 \pi}\left|\sum_{n=1,2,3,4,5} U_{2 n} U_{1 n}^{*} F\left(x_{n}\right)\right|^{2}
\end{array}
$$

where the $\alpha_{W}$ is the weak coupling constant. As for the branching ratio of $\mu \rightarrow e \gamma$ in Eq. (42), we showed that substituting $A_{\mu e}$ back into the branching ratio in Eq. (41) is exactly consistent with the one in Eq. (33). Expanding the unitary mixing matrices in the muon anomaly prediction in Eq. (42), yields the following relation:

$$
\Delta a_{\mu}=\frac{\alpha_{W}}{32 \pi} \frac{m_{\mu}^{2}}{M_{W}^{2}}\left(\left(1-2 \eta_{22}\right) F(0)+2 \eta_{22} F\left(x_{4}\right)\right)
$$

Looking at Eq. (43), it is clear that the SM part which is without $\eta$ and the BSM having $\eta$ are entangled together. We arrive at the right prediction for the muon anomaly at one-loop by removing the SM part from Eq. (43)

$$
\Delta a_{\mu}=\frac{\alpha_{W}}{16 \pi} \frac{m_{\mu}^{2}}{M_{W}^{2}} \eta_{22}\left(F\left(x_{4}\right)-F(0)\right) .
$$

Similarly to the branching ratio of $\mu \rightarrow e \gamma$ decay, it can be confirmed that the prediction for the muon anomaly also consists of the factor of deviation of unitarity $\eta$.

\section{The anomalous electron magnetic moment $g-2$}

As in the muon anomalous magnetic moment, the same diagrams with external particles replaced by electrons can be generated in Fig. 8.

Using the complete form of the muon anomaly prediction in Eq. (44), we can derive the right prediction for the electron anomalous magnetic moment with slight modifications $m_{\mu} \rightarrow m_{e}, \eta_{22} \rightarrow \eta_{11}$.

$$
\Delta a_{e}=\frac{\alpha_{W}}{16 \pi} \frac{m_{e}^{2}}{M_{W}^{2}} \eta_{11}\left(F\left(x_{4}\right)-F(0)\right) .
$$

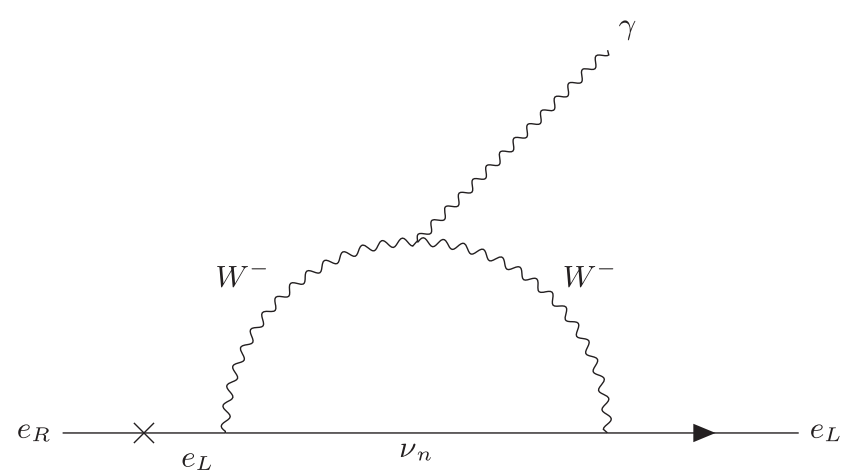

\section{E. Numerical analysis of $W$ exchange contributions}

The presence of heavy vectorlike neutrinos leads to the deviation of unitarity and the observables $\Delta a_{\mu, e}$ and constraint $\operatorname{BR}(\mu \rightarrow e \gamma)$ can be written in terms of the factor of nonunitarity $\eta$.

$$
\begin{aligned}
\operatorname{BR}(\mu \rightarrow e \gamma) & =\frac{3 \alpha_{e m}}{8 \pi}\left|\eta_{21}\right|^{2}\left(F\left(x_{4}\right)-F(0)\right)^{2} \\
\Delta a_{\mu} & =\frac{\alpha_{W}}{16 \pi} \frac{m_{\mu}^{2}}{M_{W}^{2}} \eta_{22}\left(F\left(x_{4}\right)-F(0)\right) \\
\Delta a_{e} & =\frac{\alpha_{W}}{16 \pi} \frac{m_{e}^{2}}{M_{W}^{2}} \eta_{11}\left(F\left(x_{4}\right)-F(0)\right) .
\end{aligned}
$$

\section{The branching ratio of $\mu \rightarrow$ er decay}

We consider the branching ratio of $\mu \rightarrow e \gamma$ decay first. Since we assume that mass of heavy vectorlike neutrinos are heavier than $1 \mathrm{TeV}$, the value of $F(0)$ for the light neutrinos converges to approximately 3.3 , while that of $F\left(x_{4}\right)$ for the heavy vectorlike neutrino converges to 1.3 . Therefore, the branching ratio of $\mu \rightarrow e \gamma$ decay can be reduced to[11].

$$
\begin{aligned}
\operatorname{BR}(\mu \rightarrow e \gamma) & =\frac{3 \alpha_{e m}}{8 \pi}\left|\eta_{21}\right|^{2}\left(F\left(x_{4}\right)-F(0)\right)^{2} \\
& \leq \frac{3 \alpha_{e m}}{2 \pi}\left|\eta_{21}\right|^{2} .
\end{aligned}
$$

The nonunitarity $\eta$ of Eq. (26) consists of four free parameters: mass of heavy vectorlike neutrinos $M_{44}^{\nu}$, a real number $y$, a $C P$ violation phase $\delta$, and a Majorana phase $\alpha$. The experimental branching ratio of $\mu \rightarrow e \gamma$ decay constrains the minimal parameter space in terms of $M_{44}^{\nu}$ and $y$, while setting up two phases $\delta, \alpha$ which maximize or minimize the branching ratio of $\mu \rightarrow e \gamma$ [11], and the minimal parameter space is shown in Fig. 9.

The left plot in Fig. 9 is an available parameter space for mass of the vectorlike neutrino versus the free parameter $y$ times SM up-type Higgs vev $v_{u}$. The blue bold line corresponds to bound of the branching ratio of $\mu \rightarrow e \gamma$ decay at the normal hierarchy with $C P$ violation phase $\delta=0$ and Majorana phase $\alpha=0$ and this line can be

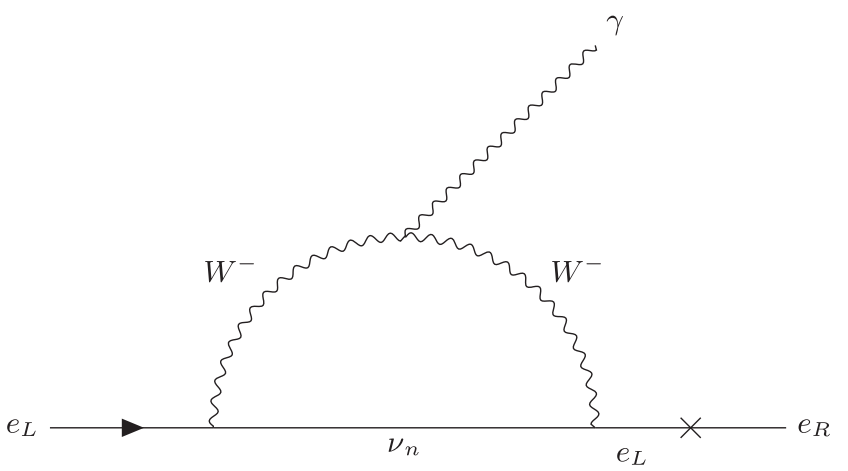

FIG. 8. Diagrams for electron anomalous magnetic moment with all neutrinos. Here $n=1,2,3,4,5$. 

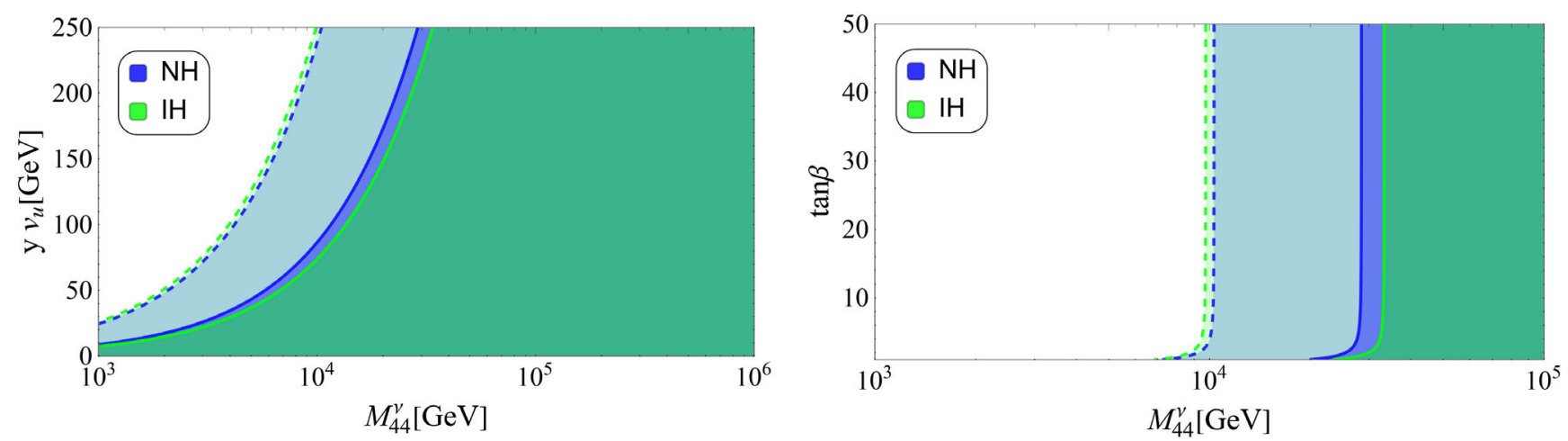

FIG. 9. The left plot is an available parameter space for two free parameters: mass of vectorlike neutrino $M_{44}^{\nu}$ and SM up-type Higgs vev $v_{u}$. Here, the free parameter $y$ is set to 1 . The right plot is the case where vev of the up-type Higgs is constrained from $246 / \sqrt{2} \simeq 174$ to $246 \mathrm{GeV}$ or from $\tan \beta=1$ to 50 in a same way.

relaxed up to the blue dotted line where $\delta=0, \alpha=2 \pi$. The green bold(dotted) line corresponds to the inverted hierarchy with $\delta=\pi / 2(0)$ and $\alpha=\frac{9 \pi}{10}(0)$. Since we are especially interested in the range of SM up-type Higgs vev $v_{u}$ from 174 to $246 \mathrm{GeV}$, the right plot consistent with the interested range is extracted from the left after replacing $v_{u}$ by $\tan \beta=v_{u} / v_{d}$ using the relation $v_{u}^{2}+v_{d}^{2}=(246 \mathrm{GeV})^{2}$.

As for the constraint of deviation of unitarity $\eta$ with the CLFV $\mu \rightarrow e \gamma$ decay at $1 \sigma$, it is given by $[86,87]$.

$$
\left|\eta_{21}\right| \leq 8.4 \times 10^{-6}
$$

\section{The muon and electron anomalous magnetic moments $\Delta a_{\mu, e}$}

As in the constraint for $\eta_{21}$ in Eq. (48), the other nonunitarities $\eta_{11,22}$ for the electron and muon anomalous magnetic moment are given by $[11,86]$.

$$
\begin{array}{ll}
\eta_{11}<4.2 \times 10^{-4}(\text { for } \mathrm{NH}), & <4.8 \times 10^{-4}(\text { for } \mathrm{IH}) \\
\eta_{22}<2.9 \times 10^{-7}(\text { for } \mathrm{NH}), & <2.4 \times 10^{-7}(\text { for } \mathrm{IH}) .
\end{array}
$$

With the constraints $\eta_{11,22}$ in Eq. (49), we can calculate impact of the muon and electron anomalous magnetic moments at $\mathrm{NH}$ (IH) using Eq. (46).

$\begin{aligned} \Delta a_{\mu} & =\frac{\alpha_{W}}{16 \pi} \frac{m_{\mu}^{2}}{M_{W}^{2}} \eta_{22}\left(F\left(x_{4}\right)-F(0)\right) \simeq-6.6(-5.5) \times 10^{-16} \\ \Delta a_{e} & =\frac{\alpha_{W}}{16 \pi} \frac{m_{e}^{2}}{M_{W}^{2}} \eta_{11}\left(F\left(x_{4}\right)-F(0)\right) \simeq-2.2(-2.6) \times 10^{-17} .\end{aligned}$

There are two interesting features in the above prediction for the muon and electron anomalous magnetic moments. One feature is sign of each prediction. As mentioned in the introduction, this prediction with the $W$ exchange can not flip the sign of each anomaly. In order to explain both anomalies at $1 \sigma$, the prediction for both anomalies with $W$ exchange requires additional contributions such as $Z^{\prime}$ or scalar exchange. Another feature is magnitude of each prediction. For the muon anomaly, the experimental order of magnitude at $1 \sigma$ is about $10^{-9}$, however our prediction is much smaller than that of the experimental bound as well as the electron anomaly, which means the nonunitarity derived from the presence of heavy vectorlike neutrino can not bring the anomalies to the observable level. This inadequate prediction with $W$ exchange has been a good motivation to search for another possibility such as scalar exchange.

\section{HIGGS EXCHANGE CONTRIBUTIONS TO $(g-2)_{\mu},(g-2)_{e}$, AND BR $(\mu \rightarrow e \gamma)$}

The relevant sector for the muon and electron anomalous magnetic moments with scalar exchange is the charged lepton Yukawa matrix which can be expressed in the mass insertion formalism as,

$$
\begin{aligned}
y_{i j}^{e}= & \left(\begin{array}{ccc}
0 & 0 & 0 \\
0 & y_{24}^{e} x_{42}^{e} & y_{24}^{e} x_{43}^{e} \\
0 & y_{34}^{e} x_{42}^{e} & y_{34}^{e} x_{43}^{e}
\end{array}\right) \frac{\langle\phi\rangle}{M_{44}^{e}} \\
& +\left(\begin{array}{lll}
y_{15}^{e} x_{51}^{e} & y_{15}^{e} x_{52}^{e} & y_{15}^{e} x_{53}^{e} \\
y_{25}^{e} x_{51}^{e} & y_{25}^{e} x_{52}^{e} & y_{25}^{e} x_{53}^{e} \\
y_{35}^{e} x_{51}^{e} & y_{35}^{e} x_{52}^{e} & y_{35}^{e} x_{53}^{e}
\end{array}\right) \frac{\langle\phi\rangle}{M_{55}^{e}} \\
& +\left(\begin{array}{lll}
y_{51}^{e} x_{15}^{L} & y_{52}^{e} x_{15}^{L} & y_{53}^{e} x_{15}^{L} \\
y_{51}^{e} x_{25}^{L} & y_{52}^{e} x_{25}^{L} & y_{53}^{e} x_{25}^{L} \\
y_{51}^{e} x_{35}^{L} & y_{52}^{e} x_{35}^{L} & y_{53}^{e} x_{35}^{L}
\end{array}\right) \frac{\langle\phi\rangle}{M_{55}^{L}} \\
& +\left(\begin{array}{lll}
0 & 0 & 0 \\
0 & 0 & 0 \\
0 & 0 & x_{34}^{L} y_{43}^{e}
\end{array}\right) \frac{\langle\phi\rangle}{M_{44}^{L}}
\end{aligned}
$$

The effective Yukawa matrix of Eq. (51) in the mass basis is diagonalized by the universal seesaw mechanism due to involving a few of different mass scales. Therefore, 

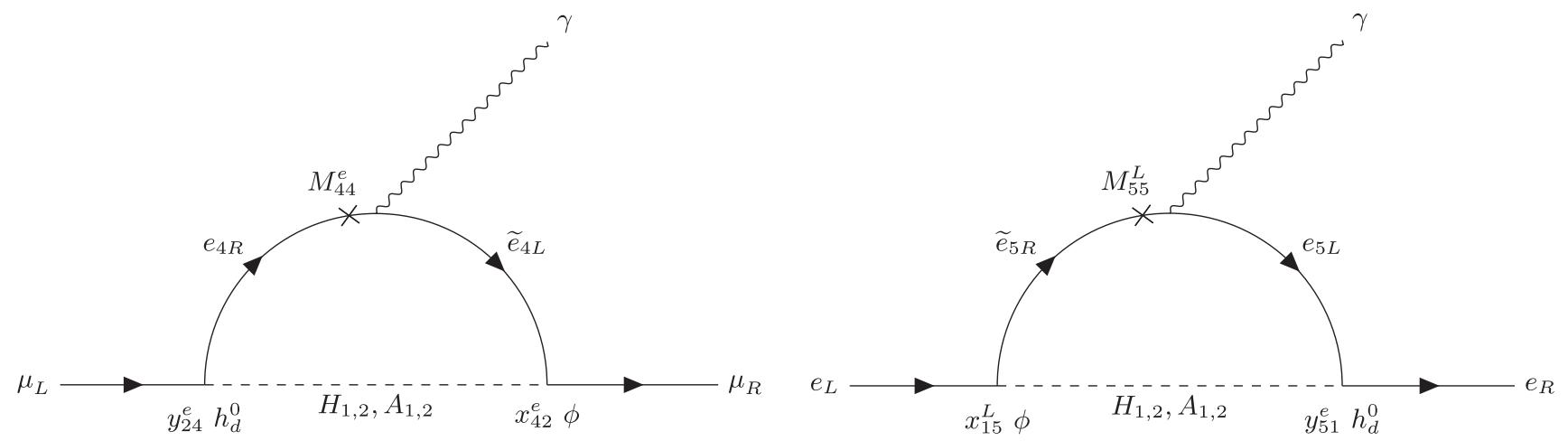

FIG. 10. Diagrams contributing to the muon anomaly (left) and the electron anomaly (right) where $H_{1,2}$ are $C P$-even non-SM scalars and $A_{1,2}$ are $C P$-odd scalars in the physical basis.

the only diagonal components should alive in the mass matrix. In order to make the mass matrix diagonal, we assume that $y_{34}^{e}=x_{43}^{e}=y_{15,25,35}^{e}=x_{51,52,53}^{e}=x_{25,35}^{L}=$ $y_{52,53}^{e}=0$. Then, the mass matrix is reduced to

$$
\begin{aligned}
y_{i j}^{e}= & \left(\begin{array}{ccc}
0 & 0 & 0 \\
0 & y_{24}^{e} x_{42}^{e} & 0 \\
0 & 0 & 0
\end{array}\right) \frac{\langle\phi\rangle}{M_{44}^{e}}+\left(\begin{array}{ccc}
0 & 0 & 0 \\
0 & 0 & 0 \\
0 & 0 & 0
\end{array}\right) \frac{\langle\phi\rangle}{M_{55}^{e}} \\
& +\left(\begin{array}{ccc}
y_{51}^{e} x_{15}^{L} & 0 & 0 \\
0 & 0 & 0 \\
0 & 0 & 0
\end{array}\right) \frac{\langle\phi\rangle}{M_{55}^{L}}+\left(\begin{array}{ccc}
0 & 0 & 0 \\
0 & 0 & 0 \\
0 & 0 & x_{34}^{L} y_{43}^{e}
\end{array}\right) \frac{\langle\phi\rangle}{M_{44}^{L}} \\
y_{i j}^{e}= & \left(\begin{array}{ccc}
y_{51}^{e} s_{15}^{L} & 0 & 0 \\
0 & y_{24}^{e} s_{24}^{e} & 0 \\
0 & 0 & y_{43}^{e} s_{34}^{L}
\end{array}\right),
\end{aligned}
$$

where $\quad s_{15}^{L} \simeq x_{15}^{L}\langle\phi\rangle / M_{55}^{L}, \quad s_{24}^{e} \simeq x_{42}^{e}\langle\phi\rangle / M_{44}^{e}, \quad s_{34}^{L} \simeq$ $x_{34}^{L}\langle\phi\rangle / M_{44}^{L}$ and the diagonal elements from top-left to bottom-right should be responsible for electron, muon and tau Yukawa constants, respectively. After removing all irrelevant terms to both anomalies and applying the assumption, the $7 \times 7$ mass matrix in the interaction basis is also reduced to as below:

$$
M^{e}=\left(\begin{array}{c|cccc} 
& e_{1 R} & e_{2 R} & e_{4 R} & \tilde{L}_{5 R} \\
\hline \bar{L}_{1 L} & 0 & 0 & 0 & x_{15}^{L} v_{\phi} \\
\bar{L}_{2 L} & 0 & 0 & y_{24}^{e} v_{d} & 0 \\
\bar{L}_{5 L} & y_{51}^{e} v_{d} & 0 & 0 & M_{55}^{L} \\
\overline{\tilde{e}}_{4 L} & 0 & x_{42}^{e} v_{\phi} & M_{44}^{e} & 0
\end{array}\right)
$$

The reduced charged lepton mass matrix of Eq. (53) clearly tells that no mixing between charged leptons arise so the branching ratio of $\mu \rightarrow e \gamma$ is naturally satisfied under this scenario. The scalar exchange for both anomalies can be realized by closing the Higgs sectors in Fig. 3 as per Fig. 10.
In Figure 10, the $C P$-even non-SM scalars $H_{1,2}$ and $C P$-odd scalars $A_{1,2}$ appear as a result of mixing between Higgses $H_{u}, H_{d}$, and $\phi$ in the interaction basis. The Higgs sector in the interaction basis is defined by

$$
\begin{aligned}
H_{u} & =\left(\begin{array}{c}
H_{u}^{+} \\
v_{u}+\frac{1}{\sqrt{2}}\left(\operatorname{Re} H_{u}^{0}+i \operatorname{Im} H_{u}^{0}\right)
\end{array}\right), \\
H_{d} & =\left(\begin{array}{c}
v_{d}+\frac{1}{\sqrt{2}}\left(\operatorname{Re} H_{d}^{0}+i \operatorname{Im} H_{d}^{0}\right) \\
H_{d}^{-}
\end{array}\right), \\
\phi & =\frac{1}{\sqrt{2}}\left(v_{\phi}+\operatorname{Re} \phi+i \operatorname{Im} \phi\right) .
\end{aligned}
$$

For consistency, we equate $v_{u}, v_{d}$ and $v_{\phi}$ to $v_{1}, v_{2}$, and $v_{3}$, respectively.

\section{A. The 2HDM scalar potential}

The scalar potential of the model under consideration takes the form:

$$
\begin{aligned}
V= & \mu_{1}^{2}\left(H_{u} H_{u}^{\dagger}\right)+\mu_{2}^{2}\left(H_{d} H_{d}^{\dagger}\right)+\mu_{3}^{2}\left(\phi \phi^{*}\right) \\
& +\mu_{s b}^{2}\left[\phi^{2}+\left(\phi^{*}\right)^{2}\right]+\lambda_{1}\left(H_{u} H_{u}^{\dagger}\right)^{2}+\lambda_{2}\left(H_{d} H_{d}^{\dagger}\right)^{2} \\
& +\lambda_{3}\left(H_{u} H_{u}^{\dagger}\right)\left(H_{d} H_{d}^{\dagger}\right)+\lambda_{4}\left(H_{u} H_{d}^{\dagger}\right)\left(H_{d} H_{u}^{\dagger}\right) \\
& +\lambda_{5}\left(\varepsilon_{i j} H_{u}^{i} H_{d}^{j} \phi^{2}+H . c\right)+\lambda_{6}\left(\phi \phi^{*}\right)^{2} \\
& +\lambda_{7}\left(\phi \phi^{*}\right)\left(H_{u} H_{u}^{\dagger}\right)+\lambda_{8}\left(\phi \phi^{*}\right)\left(H_{d} H_{d}^{\dagger}\right),
\end{aligned}
$$

where the $\lambda_{i}(i=1,2, \ldots, 8)$ are dimensionless parameters whereas the $\mu_{j}(j=1,2,3)$ are dimensionful parameters and $\mu_{s b}$ is a dimensionful soft-breaking parameter. We consider the $U(1)^{\prime}$ symmetry as global in this model so our model does not feature $Z^{\prime}$ boson and the scalar potential requires the inclusion of the soft-breaking mass term $-\mu_{s b}^{2}\left[\phi^{2}+\left(\phi^{*}\right)^{2}\right]$ in order to prevent the appearance of a massless scalar state arising from the imaginary part of $\phi$. 
The minimization conditions of the scalar potential yield the following relations:

$$
\begin{aligned}
& \mu_{1}^{2}=-2 \lambda_{1} v_{1}^{2}-\lambda_{3} v_{2}^{2}-\frac{1}{2} \lambda_{7} v_{3}^{2}+\frac{\lambda_{5} v_{2} v_{3}^{2}}{2 v_{1}}, \\
& \mu_{2}^{2}=-2 \lambda_{2} v_{2}^{2}-\lambda_{3} v_{1}^{2}-\frac{1}{2} \lambda_{8} v_{3}^{2}+\frac{\lambda_{5} v_{3}^{2} v_{1}}{2 v_{2}}, \\
& \mu_{3}^{2}=-\lambda_{8} v_{2}^{2}-\lambda_{6} v_{3}^{2}+v_{1}\left(2 \lambda_{5} v_{2}-\lambda_{7} v_{1}\right)-2 \mu_{s b}^{2} .
\end{aligned}
$$

\section{B. Mass matrix for $\boldsymbol{C P}$-even, $\boldsymbol{C P}$-odd, neutral, and charged scalars}

The squared mass matrix for the $C P$-even scalars in the basis $\left(\operatorname{Re} H_{u}^{0}, \operatorname{Re} H_{d}^{0}, \operatorname{Re} \phi\right)$ takes the form:

$$
\mathbf{M}_{C P \text {-even }}^{2}=\left(\begin{array}{ccc}
4 \lambda_{1} v_{1}^{2}+\frac{\lambda_{5} v_{2} v_{3}^{2}}{2 v_{1}} & -\frac{1}{2} \lambda_{5} v_{3}^{2}+2 \lambda_{3} v_{1} v_{2} & \sqrt{2} v_{3}\left(-\lambda_{5} v_{2}+\lambda_{7} v_{1}\right) \\
-\frac{1}{2} \lambda_{5} v_{3}^{2}+2 \lambda_{3} v_{1} v_{2} & 4 \lambda_{2} v_{2}^{2}+\frac{\lambda_{5} v_{1} v_{3}^{2}}{2 v_{2}} & \sqrt{2} v_{3}\left(-\lambda_{5} v_{1}+\lambda_{8} v_{2}\right) \\
\sqrt{2} v_{3}\left(-\lambda_{5} v_{2}+\lambda_{7} v_{1}\right) & \sqrt{2} v_{3}\left(-\lambda_{5} v_{1}+\lambda_{8} v_{2}\right) & 2 \lambda_{6} v_{3}^{2}
\end{array}\right) .
$$

From the mass matrix given above, we find that the $C P$-even scalar spectrum is composed of the $125 \mathrm{GeV}$ SM-like Higgs $h$ and two non-SM $C P$-even Higgses $H_{1,2}$. Furthermore, we assume that no mixing between the SM physical Higgs $h$ and the two non-SM $C P$-even Higgses $H_{1,2}$ arise and this assumption constrains the $(1,2),(1,3),(2,1)$, and $(3,1)$ elements of $C P$-even mass matrix of Eq. (57). The constraints are given by the following decoupling limit scenario

$$
\begin{aligned}
& \lambda_{5}=\frac{4 v_{1} v_{2}}{v_{3}^{2}} \lambda_{3} \\
& \lambda_{7}=\frac{v_{2}}{v_{1}} \lambda_{5}=\frac{4 v_{2}^{2}}{v_{3}^{2}} \lambda_{3},
\end{aligned}
$$

and then the $C P$-even mass matrix of Eq. (57) with the constraints is simplified to

$$
\mathbf{M}_{C P-\mathrm{even}}^{2}=\left(\begin{array}{ccc}
4 \lambda_{1} v_{1}^{2}+2 v_{2}^{2} \lambda_{3} & 0 & 0 \\
0 & 4 \lambda_{2} v_{2}^{2}+2 v_{1}^{2} \lambda_{3} & \sqrt{2} v_{3}\left(-\frac{4 v_{1}^{2} v_{2}}{v_{3}^{2}} \lambda_{3}+\lambda_{8} v_{2}\right) \\
0 & \sqrt{2} v_{3}\left(-\frac{4 v_{1}^{2} v_{2}}{v_{3}^{2}} \lambda_{3}+\lambda_{8} v_{2}\right) & 2 \lambda_{6} v_{3}^{2}
\end{array}\right)
$$

In the above given decoupling limit scenario, chosen in order to simplify our analysis, the $C P$-even neutral scalar states contained in the $S U(2)$ doublet $H_{u}$ will not mix with the $C P$-even neutral ones contained in $H_{d}$. In such limit, the neutral $C P$-even states of $H_{u}$ will not feature mixing with the gauge singlet scalar $\phi$. Thus, the lightest $125 \mathrm{GeV} C P$ even scalar of our model will have couplings to the SM particles close to the SM expectation, which is consistent with the current experimental data.

Diagonalizing the simplified $C P$-even mass matrix, it reveals masses of the physical SM Higgs $h$ and non-SM $C P$-even scalars $H_{1,2}$ in the physical basis $\left(h, H_{1}, H_{2}\right)$

$R_{C P-\text { even }}^{\dagger} \mathbf{M}_{C P-\text { even }}^{2} R_{C P-\text { even }}=\operatorname{diag}\left(m_{h}^{2}, m_{H_{1}}^{2}, m_{H_{2}}^{2}\right)$.

The SM Higgs $h$ is appeared as $\operatorname{Re} H_{u}^{0}$ itself and the nonSM $C P$-even scalars $H_{1,2}$ are the states which $\operatorname{Re} H_{d}^{0}$ is mixed with Re $\phi$. Regarding the $C P$-odd scalar sector, we find that the squared mass matrix for the $C P$-odd scalars in the basis $\left(\operatorname{Im} H_{u}^{0}, \operatorname{Im} H_{d}^{0}, \operatorname{Im} \phi\right)$ is given by:

$$
\mathbf{M}_{C P-\mathrm{odd}}^{2}=\left(\begin{array}{ccc}
\frac{\lambda_{5} v_{2} v_{3}^{2}}{2 v_{1}} & \frac{1}{2} \lambda_{5} v_{3}^{2} & \sqrt{2} \lambda_{5} v_{2} v_{3} \\
\frac{1}{2} \lambda_{5} v_{3}^{2} & \frac{\lambda_{5} v_{1} v_{3}^{2}}{2 v_{2}} & \sqrt{2} \lambda_{5} v_{1} v_{3} \\
\sqrt{2} \lambda_{5} v_{2} v_{3} & \sqrt{2} \lambda_{5} v_{1} v_{3} & 4 \lambda_{5} v_{1} v_{2}-4 \mu_{s b}^{2}
\end{array}\right)
$$

The squared $C P$-odd mass matrix is diagonalized in the same way as in the $C P$-even mass matrix and the $C P$-odd physical basis is given by $\left(G_{Z}, A_{1}, A_{2}\right)$ where $G_{Z}$ is the massless Goldstone bosons associated with the longitudinal components of the $Z$ gauge boson, whereas $A_{1}$ and $A_{2}$ are massive non-SM $C P$-odd scalars 


$$
R_{C P-\text { odd }}^{\dagger} \mathbf{M}_{C P \text {-odd }}^{2} R_{C P-\text { odd }}=\operatorname{diag}\left(0, m_{A_{1}}^{2}, m_{A_{2}}^{2}\right) .
$$

Furthermore, the squared mass matrix for the electrically charged scalars is given by:

$$
\mathbf{M}_{\text {charged }}^{2}=\left(\begin{array}{cc}
\lambda_{4} v_{2}^{2}+\frac{\lambda_{5} v_{2} v_{3}^{2}}{2 v_{1}} & \lambda_{4} v_{1} v_{2}+\frac{1}{2} \lambda_{5} v_{3}^{2} \\
\lambda_{4} v_{1} v_{2}+\frac{1}{2} \lambda_{5} v_{3}^{2} & \lambda_{4} v_{1}^{2}+\frac{\lambda_{5} v_{1} v_{3}^{2}}{2 v_{2}}
\end{array}\right) .
$$

The charged scalar mass matrix can be diagonalized in the basis $\left(H_{1}^{ \pm}, H_{2}^{ \pm}\right)$as in $C P$-even or -odd mass matrix:

$$
R_{\text {charged }}^{\dagger} \mathbf{M}_{\text {charged }}^{2} R_{\text {charged }}=\operatorname{diag}\left(0, m_{H^{ \pm}}^{2}\right) .
$$

Then, the electrically charged scalar sector contains the massive scalars $H^{ \pm}$and the massless electrically charged scalars $G_{W}^{ \pm}$which correspond to the Goldstone bosons associated with the longitudinal components of the $W^{ \pm}$ gauge bosons. In the following sections we will analyze the phenomenological implications of our model in the Higgs diphoton decay as well as in the muon and electron anomalous magnetic moments.

\section{The Higgs diphoton signal strength}

The rate for the $h \rightarrow \gamma \gamma$ decay is given by:

$$
\begin{aligned}
\Gamma(h \rightarrow \gamma \gamma)= & \frac{\alpha_{e m}^{2} m_{h}^{3}}{256 \pi^{3} v^{2}} \mid \sum_{f} a_{h f f} N_{C} Q_{f}^{2} F_{1 / 2}\left(\rho_{f}\right) \\
& +a_{h W W} F_{1}\left(\rho_{W}\right)+\left.\frac{C_{h H^{ \pm} H^{\mp}} v}{2 m_{H^{ \pm}}^{2}} F_{0}\left(\rho_{H_{k}^{ \pm}}\right)\right|^{2},
\end{aligned}
$$

where $\rho_{i}$ are the mass ratios $\rho_{i}=\frac{m_{h}^{2}}{4 M_{i}^{2}}$ with $M_{i}=m_{f}, M_{W}$; $\alpha_{e m}$ is the fine structure constant; $N_{C}$ is the color factor ( $N_{C}=1$ for leptons and $N_{C}=3$ for quarks) and $Q_{f}$ is the electric charge of the fermion in the loop. From the fermion-loop contributions we only consider the dominant top quark term. Furthermore, $C_{h H^{ \pm} H^{\mp}}$ is the trilinear coupling between the SM-like Higgs and a pair of charged Higges, whereas $a_{h t t}$ and $a_{h W W}$ are the deviation factors from the SM Higgs-top quark coupling and the SM Higgs$\mathrm{W}$ gauge boson coupling, respectively (in the SM these factors are unity). Such deviation factors are close to unity in our model and they are defined as below:

$$
\begin{aligned}
a_{h t t} & \simeq 1, a_{h W W} \\
& =\frac{1}{\sqrt{v_{1}^{2}+v_{2}^{2}}} \frac{\partial}{\partial h}\left(\sum_{i, j=1,2,3} v_{i}\left(R_{C P-\mathrm{even}}^{T}\right)_{i j}\left(h, H_{1}, H_{2}\right)_{j}\right) \\
& =\frac{v_{1}}{\sqrt{v_{1}^{2}+v_{2}^{2}}}
\end{aligned}
$$

Furthermore, $F_{1 / 2}(z)$ and $F_{1}(z)$ are the dimensionless loop factors for spin- $1 / 2$ and spin- 1 particles running in the internal lines of the loops. These loop factors take the form:

$$
\begin{aligned}
F_{1 / 2}(z) & =2(z+(z-1) f(z)) z^{-2}, \\
F_{1}(z) & =-2\left(2 z^{2}+3 z+3(2 z-1) f(z)\right) z^{-2}, \\
F_{0}(z) & =-(z-f(z)) z^{-2}
\end{aligned}
$$

with

$f(z)=\left\{\begin{array}{lll}\arcsin ^{2} \sqrt{2} & \text { for } & z \leq 1 \\ -\frac{1}{4}\left(\ln \left(\frac{1+\sqrt{1-z^{-1}}}{1-\sqrt{1-z^{-1}}-i \pi}\right)^{2}\right) & \text { for } & z>1\end{array}\right.$

In order to study the implications of our model in the decay of the $125 \mathrm{GeV}$ Higgs into a photon pair, one introduces the Higgs diphoton signal strength $R_{\gamma \gamma}$, which is defined as:

$$
\begin{aligned}
R_{\gamma \gamma} & =\frac{\sigma(p p \rightarrow h) \Gamma(h \rightarrow \gamma \gamma)}{\sigma(p p \rightarrow h)_{\mathrm{SM}} \Gamma(h \rightarrow \gamma \gamma)_{\mathrm{SM}}} \\
& \simeq a_{h t t}^{2} \frac{\Gamma(h \rightarrow \gamma \gamma)}{\Gamma(h \rightarrow \gamma \gamma)_{\mathrm{SM}}} .
\end{aligned}
$$

That Higgs diphoton signal strength, normalizes the $\gamma \gamma$ signal predicted by our model in relation to the one given by the SM. Here we have used the fact that in our model, single Higgs production is also dominated by gluon fusion as in the Standard Model.

The ratio $R_{\gamma \gamma}$ has been measured by CMS and ATLAS collaborations with the best fit signals $[88,89]$ :

$R_{\gamma \gamma}^{\mathrm{CMS}}=1.18_{-0.14}^{+0.17} \quad$ and $\quad R_{\gamma \gamma}^{\mathrm{ATLAS}}=0.96 \pm 0.14$.

As it will be shown in the next subsection, the constraints arising from the Higgs diphoton decay rate will be considered in our numerical analysis.

\section{The muon and electron anomalous magnetic moments}

The Yukawa interactions relevant for the computation of the muon anomalous magnetic moment are

$$
\begin{aligned}
\mathcal{L}_{\Delta a_{\mu}}= & y_{24}^{e} \mu\left(\operatorname{Re} H_{d}^{0}-i \gamma^{5} \operatorname{Im} H_{d}^{0}\right) \bar{e}_{4} \\
& +x_{42}^{e} \tilde{e}_{4}\left(\operatorname{Re} \phi-i \gamma^{5} \operatorname{Im} \phi\right) \bar{e}_{2}+M_{44}^{e} \tilde{e}_{4} \bar{e}_{4}+\text { H.c. }
\end{aligned}
$$

where the Yukawa coupling constants $y_{24}^{e}, x_{42}^{e}$ are assumed to be real, the scalar fields have been expanded by their real and imaginary parts and the properties of the projection operators $P_{L, R}$ acting on the charged leptonic fields have been used.

By expressing the scalar fields in the interaction basis in terms of the scalar fields in the physical basis, the charged lepton Yukawa interactions relevant for the computation of the $g-2$ anomalies take the form: 


$$
\begin{aligned}
\mathcal{L}_{\Delta a_{\mu}}= & y_{24}^{e} \mu\left(\left(R_{e}^{T}\right)_{22} H_{1}+\left(R_{e}^{T}\right)_{23} H_{2}\right. \\
& \left.-i \gamma^{5}\left(R_{o}^{T}\right)_{22} A_{1}-i \gamma^{5}\left(R_{o}^{T}\right)_{23} A_{2}\right) \bar{e}_{4} \\
& +x_{42}^{e} \tilde{e}_{4}\left(\left(R_{e}^{T}\right)_{32} H_{1}+\left(R_{e}^{T}\right)_{33} H_{2}-i \gamma^{5}\left(R_{o}^{T}\right)_{32} A_{1}\right. \\
& \left.-i \gamma^{5}\left(R_{o}^{T}\right)_{33} A_{2}\right) \bar{e}_{2}+M_{44}^{e} \tilde{e}_{4} \bar{e}_{4}+\text { H.c. }
\end{aligned}
$$

where we are using the unitary gauge where the contributions arising from unphysical Goldstone bosons to the muon anomaly are excluded and we shorten the notations $R_{C P}$ by $R_{e(o)}$. Here $R_{e}$ and $R_{o}$ are the rotation matrices that diagonalize the squared mass matrices for the $C P$ even and $C P$ odd scalars, respectively. Then, it follows that the muon and electron anomalous magnetic moments in the scenario of diagonal SM charged lepton mass matrix take the form:

$$
\begin{aligned}
\Delta a_{\mu}= & y_{24}^{e} x_{42}^{e} \frac{m_{\mu}^{2}}{8 \pi^{2}}\left[\left(R_{e}^{T}\right)_{22}\left(R_{e}^{T}\right)_{32} I_{S}^{(\mu)}\left(m_{e_{4}}, m_{H_{1}}\right)+\left(R_{e}^{T}\right)_{23}\left(R_{e}^{T}\right)_{33} I_{S}^{(\mu)}\left(m_{e_{4}}, m_{H_{2}}\right)\right. \\
& \left.-\left(R_{o}^{T}\right)_{22}\left(R_{o}^{T}\right)_{32} I_{P}^{(\mu)}\left(m_{e_{4}}, m_{A_{1}}\right)-\left(R_{o}^{T}\right)_{23}\left(R_{o}^{T}\right)_{33} I_{P}^{(\mu)}\left(m_{e_{4}}, m_{A_{2}}\right)\right] \\
\Delta a_{e}= & y_{51}^{e} x_{15}^{L} \frac{m_{e}^{2}}{8 \pi^{2}}\left[\left(R_{e}^{T}\right)_{22}\left(R_{e}^{T}\right)_{32} I_{S}^{(e)}\left(m_{e_{5}}, m_{H_{1}}\right)+\left(R_{e}^{T}\right)_{23}\left(R_{e}^{T}\right)_{33} I_{S}^{(e)}\left(m_{e_{5}}, m_{H_{2}}\right)\right. \\
& \left.-\left(R_{o}^{T}\right)_{22}\left(R_{o}^{T}\right)_{32} I_{P}^{(e)}\left(m_{e_{5}}, m_{A_{1}}\right)-\left(R_{o}^{T}\right)_{23}\left(R_{e}^{T}\right)_{33} I_{P}^{(E)}\left(m_{e_{5}}, m_{A_{2}}\right)\right],
\end{aligned}
$$

where the loop integrals are given by $[85,90-93]$ :

$$
I_{S(P)}^{(e, \mu)}\left(m_{E_{4,5}}, m_{S}\right)=\int_{0}^{1} \frac{x^{2}\left(1-x \pm \frac{m_{E_{4,5}}}{m_{e, \mu}}\right)}{m_{e, \mu}^{2} x^{2}+\left(m_{E_{4,5}}^{2}-m_{e, \mu}^{2}\right) x+m_{S, P}^{2}(1-x)} d x
$$

and $S(P)$ means scalar (pseudoscalar) and $E_{4.5}$ stands for the vectorlike family. It is worth mentioning that $E_{4}$ and $E_{5}$ only contribute to the muon and electron anomalous magnetic moments, respectively.

\section{NUMERICAL ANALYSIS OF THE HIGGS EXCHANGE CONTRIBUTIONS}

For the sake of simplicity, we consider the scenario of absence of mixing between SM charged leptons, which automatically prevents charged lepton flavor violating decays. In our numerical analysis we have found that the non-SM $C P$-even scalar mass can reach values around $200 \mathrm{GeV}$. Despite the fact that the non-SM $C P$-even scalar is quite light and can have a sizeable decay mode into a bottom-antibottom quark pair, its single LHC production via gluon fusion mechanism is strongly suppressed since it is dominated by the triangular bottom quark loop. Such non-SM $C P$-even scalar $H$ can also be produced by vector boson fusion but such production is expected to have a low total cross section due to small $H W W$ and $H Z Z$ couplings, which are proportional to $v_{d}$. In this section we will discuss the implications of our model in the muon and electron anomalous magnetic moments.

\section{A. The fitting function $\chi^{\mathbf{2}}$ and free parameter setup}

For the first approach to both anomalies, we construct the fitting function $\chi^{2}$

$$
\begin{aligned}
\chi^{2}= & \frac{\left(m_{h}^{\text {Thy }}-m_{h}^{\mathrm{Cen}}\right)^{2}}{\left(\delta m_{h}^{\mathrm{Dev}}\right)^{2}}+\frac{\left(a_{h W W}^{\text {Thy }}-a_{h W W}^{\mathrm{Cen}}\right)^{2}}{\left(\delta a_{h W W}^{\mathrm{Dev}}\right)^{2}}+\frac{\left(R_{\gamma \gamma}^{\mathrm{Thy}}-R_{\gamma \gamma}^{\mathrm{Cen}}\right)^{2}}{\left(\delta R_{\gamma \gamma}^{\mathrm{Dev}}\right)^{2}} \\
& +\frac{\left(\Delta a_{\mu}^{\mathrm{Thy}}-\Delta a_{\mu}^{\mathrm{Cen}}\right)^{2}}{\left(\delta \Delta a_{\mu}^{\mathrm{Dev}}\right)^{2}}+\frac{\left(\Delta a_{e}^{\mathrm{Thy}}-\Delta a_{e}^{\mathrm{Cen}}\right)^{2}}{\left(\delta \Delta a_{e}^{\mathrm{Dev}}\right)^{2}}
\end{aligned}
$$

where the superscripts Thy, Cen and Dev mean theoretical prediction, central value of experimental bound and deviation from the central value at one of $1,2,3 \sigma$, respectively. The parameters used in this fitting function are defined as below (the integer number multiplied in delta terms means $\sigma$ ):

$$
\begin{aligned}
m_{h}^{\mathrm{Cen}} & =125.38 \mathrm{GeV}, \quad \delta m_{h}^{\mathrm{Dev}}=3 \times 0.14 \mathrm{GeV}, \\
a_{h W W}^{\mathrm{Cen}} & =0.59, \quad \delta a_{h W W}^{\mathrm{Dev}}=1 \times 0.35, \\
R_{\gamma \gamma}^{\mathrm{Cen}} & =\frac{1}{2}\left(R_{\gamma \gamma}^{\mathrm{CMS}}+R_{\gamma \gamma}^{\mathrm{ATLAS}}\right)=1.07, \quad \delta R_{\gamma \gamma}^{\mathrm{Dev}}=1 \times 0.14, \\
\Delta a_{\mu}^{\mathrm{Cen}} & =26.1 \times 10^{-10}, \quad \delta \Delta a_{\mu}^{\mathrm{Dev}}=1 \times\left(8.0 \times 10^{-10}\right) \\
\Delta a_{e}^{\mathrm{Cen}} & =-0.88 \times 10^{-12}, \quad \delta \Delta a_{e}^{\mathrm{Dev}}=2 \times\left(0.36 \times 10^{-12}\right)
\end{aligned}
$$

For an initial scan, we set up the starting parameter region as below:

(1) For the Higgs vevs, we are interested in the range of $\tan \beta$ from 5 to 50 as in the $W$ boson exchange in Fig. 9.

(2) For $\lambda_{1}$, we fixed mass of the SM physical Higgs $h$ to be $125 \mathrm{GeV}$ to save time and to make the calculation 
faster. For $\lambda_{5,7}$, the assumption that no mixing between the SM Higgs $h$ and non-SM Higgses $H_{1,2}$ arise is reflected on these parameters. All quartic coupling constants $\lambda_{1, \ldots, 8}$ are set up not to go over $4 \pi$ for perturbativity.

(3) For the vectorlike masses $M_{44}^{e}$ and $M_{55}^{L}$, there is a constraint that the lightest should be greater than $200 \mathrm{GeV}$ [94].

(4) In our numerical analysis we consider solutions where the non-SM scalar masses are larger than about $200 \mathrm{GeV}$ as done in [95].

(5) The soft-breaking mass term $\mu_{s b}$ is a free parameter, which does not generate any problem and appropriate values of this parameters yields masses for scalars and vectorlike fermions consistent with the experimental constraints.

(6) The diagonal Yukawa constants appearing in Eq. (52) should be the Yukawa constant for electron, muon, and tau, respectively. The Yukawa constants $y_{24,51}$ and $x_{42,15}$ interacting with vectorlike families are defined under this consideration. For perturbativity, the Yukawa constants $y_{24,51}$ are considered not to go over $\sqrt{4 \pi}$.

After saturating value of the $\chi^{2}$ function less than or nearly 2 which we believe it is converged enough, we find a

TABLE II. Next parameter setup after the initial scan result.

\begin{tabular}{lc}
\hline \hline Parameter & Value/Scanned Region $(\mathrm{GeV})$ \\
\hline$v_{u}=v_{1}$ & $\frac{\tan \beta_{p}}{\sqrt{1+\tan \beta_{p}^{2}}} \times 246$ \\
$v_{d}=v_{2}$ & $\frac{1}{\sqrt{1+\tan \beta_{p}^{2}}} \times 246$ \\
$v_{\phi}=v_{3}$ & {$[(1-\kappa),(1+\kappa)] \times v_{3 p}$} \\
$\tan \beta=v_{u} / v_{d}$ & {$[(1-\kappa),(1+\kappa)] \times \tan \beta_{p}$} \\
$\lambda_{1}$ & $\left(m_{h}^{2}-\frac{v_{2} v_{2}^{2} \lambda_{5}}{2 v_{1}} /\left(4 v_{1}^{2}\right)\right.$ \\
$\lambda_{2}$ & {$[(1-\kappa),(1+\kappa)] \times \lambda_{2 p}$} \\
$\lambda_{3}$ & {$[(1-\kappa),(1+\kappa)] \times \lambda_{3 p}$} \\
$\lambda_{4}$ & {$[(1-\kappa),(1+\kappa)] \times \lambda_{4 p}$} \\
$\lambda_{5}$ & $4 v_{1} v_{2} \lambda_{3} /\left(v_{3}\right)^{2}$ \\
$\lambda_{6}$ & {$[(1-\kappa),(1+\kappa)] \times \lambda_{6 p}$} \\
$\lambda_{7}$ & $v_{2} \lambda_{5} / v_{1}$ \\
$\lambda_{8}$ & {$[(1-\kappa),(1+\kappa)] \times \lambda_{8 p}$} \\
$M_{44}^{e}$ & {$[(1-\kappa),(1+\kappa)] \times M_{44 p}^{e}$} \\
$M_{55}^{L}$ & {$[(1-\kappa),(1+\kappa)] \times M_{55 p}^{L}$} \\
$\mu_{s b}$ & {$[(1-\kappa),(1+\kappa)] \times \mu_{s b p}$} \\
$y_{e}$ & $\sqrt{2} m_{e} / v_{2}$ \\
$y_{\mu}$ & $\sqrt{2} m_{\mu} / v_{2}$ \\
$y_{24}^{e}=y_{2}$ & {$[(1-\kappa),(1+\kappa)] \times y_{24 p}^{e}$} \\
$y_{51}^{e}=y_{1}$ & {$[(1-\kappa),(1+\kappa)] \times y_{51 p}^{e}$} \\
$x_{42}^{e}=x_{2}$ & $y_{\mu} M_{44}^{e} /\left(y_{24}^{e} v_{3}\right)$ \\
$x_{15}^{L}=x_{1}$ & $y_{e} M_{55}^{L} /\left(y_{51}^{e} v_{3}\right)$ \\
$\kappa$ & 0.1 \\
\hline \hline
\end{tabular}

best peaked value for each free parameter. For the given parameters, we rename them by adding an index " $p$ " to the end of subscript of each parameter like $\tan \beta_{p}$ and then the expansion factor $\kappa$ is multiplied to find a correlation between the observables and the mass parameters. Then, the parameter region is refreshed by both the specific value of each parameter and the expansion factor $\kappa$ as per Table II.

\section{B. A scanned result on the free parameters as well as observables across over the first and second scan}

The best peaked value for each parameter is listed in Table III and energy scale is in unit of GeV. Note that all

TABLE III. A best peaked value for each parameter at each case. All energy scale is in $\mathrm{GeV}$ units. Notice that in all cases $v_{3}$ is smaller than the vector like mass parameters $M_{44}^{e}$ and $M_{55}^{L}$, which is consistent with the assumption made in Sec. I, regarding the fact that the corresponding expansion parameter $v_{3} / M_{\psi}$ is less than unity.

\begin{tabular}{lccccc}
\hline \hline Parameter & case A & case B & case C & case D & case E \\
\hline$v_{u}=v_{1}$ & 245.925 & 245.936 & 245.951 & 245.917 & 245.948 \\
$v_{d}=v_{2}$ & 6.086 & 5.595 & 4.921 & 6.387 & 5.077 \\
$v_{\phi}=v_{3}$ & -57.761 & -36.470 & -57.919 & -30.746 & -17.146 \\
$\tan \beta=v_{u} / v_{d}$ & 40.410 & 43.957 & 49.977 & 38.503 & 48.441 \\
$\lambda_{1}$ & 0.063 & 0.064 & 0.066 & 0.064 & 0.065 \\
$\lambda_{2}$ & -7.978 & 8.414 & -2.000 & 2.948 & 10.382 \\
$\lambda_{3}$ & -6.344 & -2.675 & 6.242 & -1.724 & -0.706 \\
$\lambda_{4}$ & 1.859 & 2.158 & -3.633 & 10.837 & -2.796 \\
$\lambda_{5}$ & -11.384 & -11.070 & 9.009 & -11.460 & -12.000 \\
$\lambda_{6}$ & 2.888 & 1.228 & 0.866 & 1.351 & 1.324 \\
$\lambda_{7}$ & -0.282 & -0.252 & 0.180 & -0.298 & -0.248 \\
$\lambda_{8}$ & -1.363 & -1.346 & -10.845 & -11.510 & 7.033 \\
$M_{44}^{e}$ & 1475.010 & 1355.470 & 1495.770 & 1134.340 & 1681.760 \\
$M_{55}^{L}$ & 279.386 & 211.263 & 204.706 & 323.292 & 331.462 \\
$\mu_{s b}$ & $424.618 i$ & $443.435 i$ & 480.993 & $480.062 i$ & 491.533 \\
$y_{e}\left[10^{-4}\right]$ & 1.135 & 1.234 & 1.403 & 1.081 & 1.360 \\
$y_{\mu}\left[10^{-2}\right]$ & 2.391 & 2.600 & 2.956 & 2.278 & 2.865 \\
$y_{24}^{e}=y_{2}$ & -3.161 & -3.101 & -2.942 & -1.548 & 1.662 \\
$y_{51}^{e}=y_{1}$ & 2.315 & 2.164 & 2.050 & 1.352 & 3.377 \\
$x_{42}^{e}=x_{2}$ & 0.193 & 0.312 & 0.260 & 0.543 & 1.691 \\
$x_{15}^{L}=x_{1}\left[10^{-4}\right]$ & 2.371 & 3.304 & 2.419 & 8.408 & 7.787 \\
$m_{H_{1}}$ & 213.390 & 222.924 & 212.147 & 238.523 & 205.477 \\
$m_{H_{2}}$ & 911.585 & 614.516 & 891.413 & 518.147 & 354.709 \\
$m_{A_{1}}$ & 741.343 & 537.111 & 807.268 & 435.887 & 282.964 \\
$m_{A_{2}}$ & 1003.790 & 939.553 & 1035.800 & 1006.240 & 1015.760 \\
$m_{H^{ \pm}}$ & 938.259 & 674.054 & 987.625 & 929.786 & 504.684 \\
$\Delta a_{\mu}\left[10^{-9}\right]$ & 2.734 & 2.688 & 2.935 & 2.891 & 2.393 \\
$\Delta a_{e}\left[10^{-13}\right]$ & -5.073 & -8.310 & -5.543 & -6.365 & -9.232 \\
$a_{h W W}$ & 1.000 & 1.000 & 1.000 & 1.000 & 1.000 \\
$R_{\gamma \gamma}$ & 0.999 & 0.999 & 0.999 & 0.999 & 0.999 \\
$\chi^{2}$ & 1.794 & 1.516 & 1.870 & 1.740 & 1.579 \\
\hline \hline & & & & & \\
\hline
\end{tabular}


TABLE IV. A scanned range of each parameter at case A, B, C, D and E. $H_{1,2}$ mean non SM $C P$-even scalars and $A_{1,2}$ are non SM $C P$ odd scalars and $H^{ \pm}$stand for non SM charged scalars in this model. All data of $\Delta a_{\mu, e}$ are collected within the $1 \sigma$ constraint of each anomaly.

\begin{tabular}{lcccccc}
\hline \hline Parameter & case A & case B & case C & case D & case E \\
\hline$v_{u}=v_{1}$ & {$[245.907 \rightarrow 245.938]$} & {$[245.921 \rightarrow 245.947]$} & {$[245.939 \rightarrow 245.959]$} & {$[245.898 \rightarrow 245.931]$} & {$[245.935 \rightarrow 245.957]$} \\
$v_{d}=v_{2}$ & {$[5.533 \rightarrow 6.761]$} & {$[5.087 \rightarrow 6.216]$} & {$[4.474 \rightarrow 5.468]$} & {$[5.807 \rightarrow 7.096]$} & {$[4.616 \rightarrow 5.641]$} \\
$v_{\phi}=v_{3}$ & {$[-63.525 \rightarrow-51.985]$} & {$[-40.117 \rightarrow-32.823]$} & {$[-63.706 \rightarrow-52.128]$} & {$[-33.820 \rightarrow-27.671]$} & {$[-18.860 \rightarrow-15.438]$} \\
$\tan \beta=v_{u} / v_{d}$ & {$[36.371 \rightarrow 44.451]$} & {$[39.561 \rightarrow 48.353]$} & {$[44.980 \rightarrow 54.975]$} & {$[34.653 \rightarrow 42.354]$} & {$[43.597 \rightarrow 53.284]$} \\
$m_{H_{1}}$ & {$[200.000 \rightarrow 242.653]$} & {$[201.520 \rightarrow 246.046]$} & {$[200.000 \rightarrow 230.754]$} & {$[215.523 \rightarrow 261.920]$} & {$[200.000 \rightarrow 220.017]$} \\
$m_{H_{2}}$ & {$[752.061 \rightarrow 1088.130]$} & {$[516.289 \rightarrow 724.997]$} & {$[735.831 \rightarrow 1059.900]$} & {$[441.371 \rightarrow 604.981]$} & {$[338.724 \rightarrow 374.424]$} \\
$m_{A_{1}}$ & {$[638.813 \rightarrow 853.637]$} & {$[442.527 \rightarrow 640.705]$} & {$[670.550 \rightarrow 945.705]$} & {$[357.697 \rightarrow 516.760]$} & {$[266.086 \rightarrow 297.589]$} \\
$m_{A_{2}}$ & {$[892.847 \rightarrow 1141.780]$} & {$[847.825 \rightarrow 1032.140]$} & {$[927.768 \rightarrow 1154.640]$} & {$[907.576 \rightarrow 1105.770]$} & {$[918.667 \rightarrow 1114.960]$} \\
$m_{H^{ \pm}}$ & {$[783.823 \rightarrow 1111.600]$} & {$[580.316 \rightarrow 779.945]$} & {$[842.585 \rightarrow 1143.880]$} & {$[856.237 \rightarrow 1007.360]$} & {$[478.900 \rightarrow 529.178]$} \\
$M_{44}^{e}$ & {$[1327.510 \rightarrow 1622.510]$} & {$[1219.930 \rightarrow 1491.020]$} & {$[1346.190 \rightarrow 1645.330]$} & {$[1029.900 \rightarrow 1247.770]$} & {$[1513.590 \rightarrow 1849.930]$} \\
$M_{55}^{L}$ & {$[251.447 \rightarrow 307.323]$} & {$[200.000 \rightarrow 232.389]$} & {$[200.000 \rightarrow 225.176]$} & {$[290.963 \rightarrow 355.621]$} & {$[298.317 \rightarrow 364.604]$} \\
$\left|\mu_{s b}\right|$ & {$[382.158 \rightarrow 467.079]$} & {$[399.091 \rightarrow 487.777]$} & {$[432.895 \rightarrow 529.091]$} & {$[432.059 \rightarrow 528.067]$} & {$[442.381 \rightarrow 540.679]$} \\
$\Delta a_{\mu}\left[10^{-9}\right]$ & {$[1.811 \rightarrow 3.410]$} & {$[1.810 \rightarrow 3.410]$} & {$[1.810 \rightarrow 3.410]$} & {$[1.810 \rightarrow 3.410]$} & {$[1.810 \rightarrow 3.410]$} \\
$\Delta a_{e}\left[10^{-13}\right]$ & {$[-6.730 \rightarrow-5.200]$} & {$[-11.142 \rightarrow-5.985]$} & {$[-7.207 \rightarrow-5.200]$} & {$[-8.721 \rightarrow-5.200]$} & {$[-12.393 \rightarrow-5.442]$} \\
$a_{h W W}$ & {$[1.000 \rightarrow 1.000]$} & {$[1.000 \rightarrow 1.000]$} & {$[0.999 \rightarrow 1.000]$} & {$[1.000 \rightarrow 1.000]$} & {$[1.000 \rightarrow 1.000]$} \\
$R_{\gamma \gamma}$ & {$[0.999 \rightarrow 0.999]$} & {$[0.999 \rightarrow 0.999]$} & {$[0.999 \rightarrow 1.000]$} & {$[1.000 \rightarrow 1.000]$} & {$[0.999 \rightarrow 1.000]$} \\
$\chi^{2}$ & {$[1.604 \rightarrow 2.750]$} & {$[1.501 \rightarrow 2.635]$} & {$[1.580 \rightarrow 2.761]$} & {$[1.509 \rightarrow 2.749]$} & {$[1.501 \rightarrow 2.720]$} \\
\hline \hline
\end{tabular}

cases are carried out independently and all points of plots in each case are collected within $1 \sigma$ constraint of each anomaly.

Here, we put two constraints on the lightest vectorlike mass and the lightest non-SM scalar mass; the vectorlike mass should be greater than $200 \mathrm{GeV}$ as well as the non-SM scalar mass [94,95]. After we carry out second parameter scan based on the first scan result of Table III, range of the parameters are given in Table IV.

\section{The muon and electron anomalous magnetic moments}

In order to confirm that our theoretical prediction for both anomalies can accommodate their constraints at $1 \sigma$ and to analyze correlations between both anomalies and mass parameters, we consider cases B and E in Table III since their benchmark point have relative lower values of the $\chi^{2}$ function when compared to other cases. The reason that the cases $\mathrm{B}$ and $\mathrm{E}$ have the lower values of the $\chi^{2}$ function arises from the obtained value of the electron anomaly, which is very close to the central experimental value. All cases reveal nearly central value of muon anomaly constraint at $1 \sigma$, whereas the other cases except $\mathrm{B}$ and $\mathrm{E}$ reveal nearly edge value of electron anomaly constraint at $1 \sigma$. Therefore, the reason why the cases B and $\mathrm{E}$ are more converged is related to whether our theoretical prediction for both anomalies can gain access to their central value of each anomaly constraint at $1 \sigma$. More importantly, the case $\mathrm{E}$ is only one satisfying vacuum stability conditions and a detailed investigation for the vacuum stability of each case will be studied in a subsection. For these reasons, we take the case E in Table IV to study the correlations. The relevant parameter spaces are listed in Figs. 11 and 12.

To begin with, we consider the parameter spaces for the muon anomaly versus electron anomaly with a mass parameter which attends both anomalies $\left(H_{1,2}, A_{1,2}\right)$ and does not $\left(H^{ \pm}\right)$in Fig. 11. Even thought the non-SM charged scalar does not attend both anomalies, the similar pattern which the other scalars implement in Fig. 11 is also appeared. We confirmed that mass of $\mathrm{H}_{2}$ is nearly proportional to that of $H^{ \pm}$, which causes the correlation identified in plots of the other non-SM scalars in Fig. 11 is still maintained for the non-SM charged scalar. Interestingly, the cases $\mathrm{A}, \mathrm{B}$ and $\mathrm{C}$ in Table IV reported $m_{\mathrm{H}_{2}}$ is nearly proportional to $m_{H^{ \pm}}$one-to-one ratio, whereas the cases $\mathrm{D}$ and $\mathrm{E}$ revealed a fat proportion between them and still maintained the correlation.

As mentioned at the beginning of this section, we take the case $\mathrm{E}$ for the plots in Figs. 11 and 12 and a main distinction between the case $\mathrm{E}$ and others arises from the value of electron anomaly. If we take other cases instead of the case $\mathrm{E}$ to investigate the parameter spaces, the parameter region appeared in top-left plot of Fig. 11 will be shifted upward by locating at the value of -5 or $-6 \times 10^{-13}$ for the electron anomaly. In other words, the whole colored region in Fig. 11 is shifted upwards to meet the scanned value of electron anomaly constraint at $1 \sigma$, holding the correlations. Therefore, the white region appeared in Fig. 11 is not strictly excluded region and affected by 

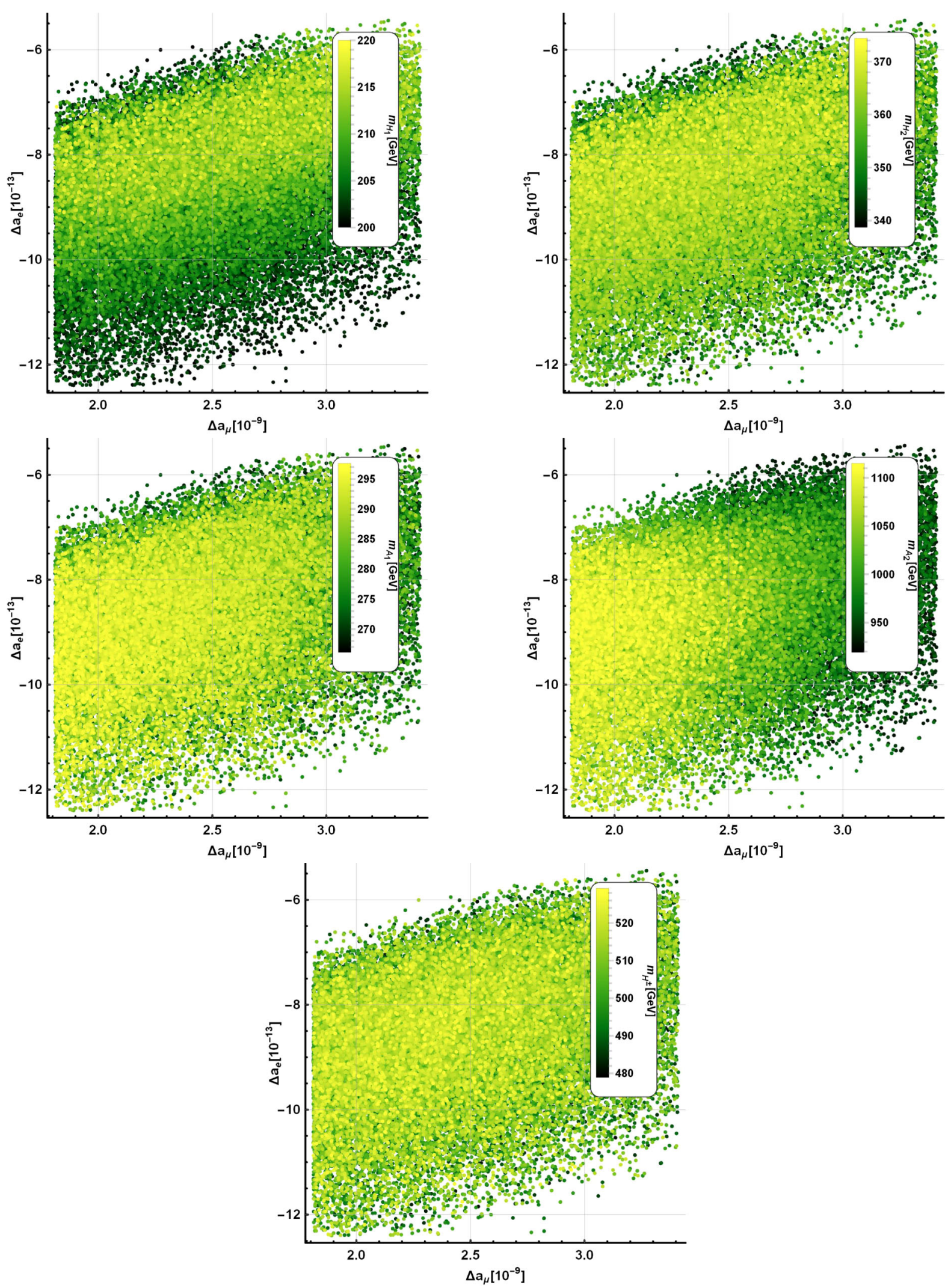

FIG. 11. Available parameter spaces for the muon anomaly versus electron anomaly with a mass parameter which attends the both anomalies $\left(H_{1,2}, A_{1,2}\right)$ and does not $\left(H^{ \pm}\right) . H_{1,2}$ are non-SM $C P$ even scalars, $A_{1,2}$ are non-SM $C P$ odd scalars and $H^{ \pm}$are non-SM charged scalars. All points in each plot are collected within $1 \sigma$ constraint of each anomaly. 

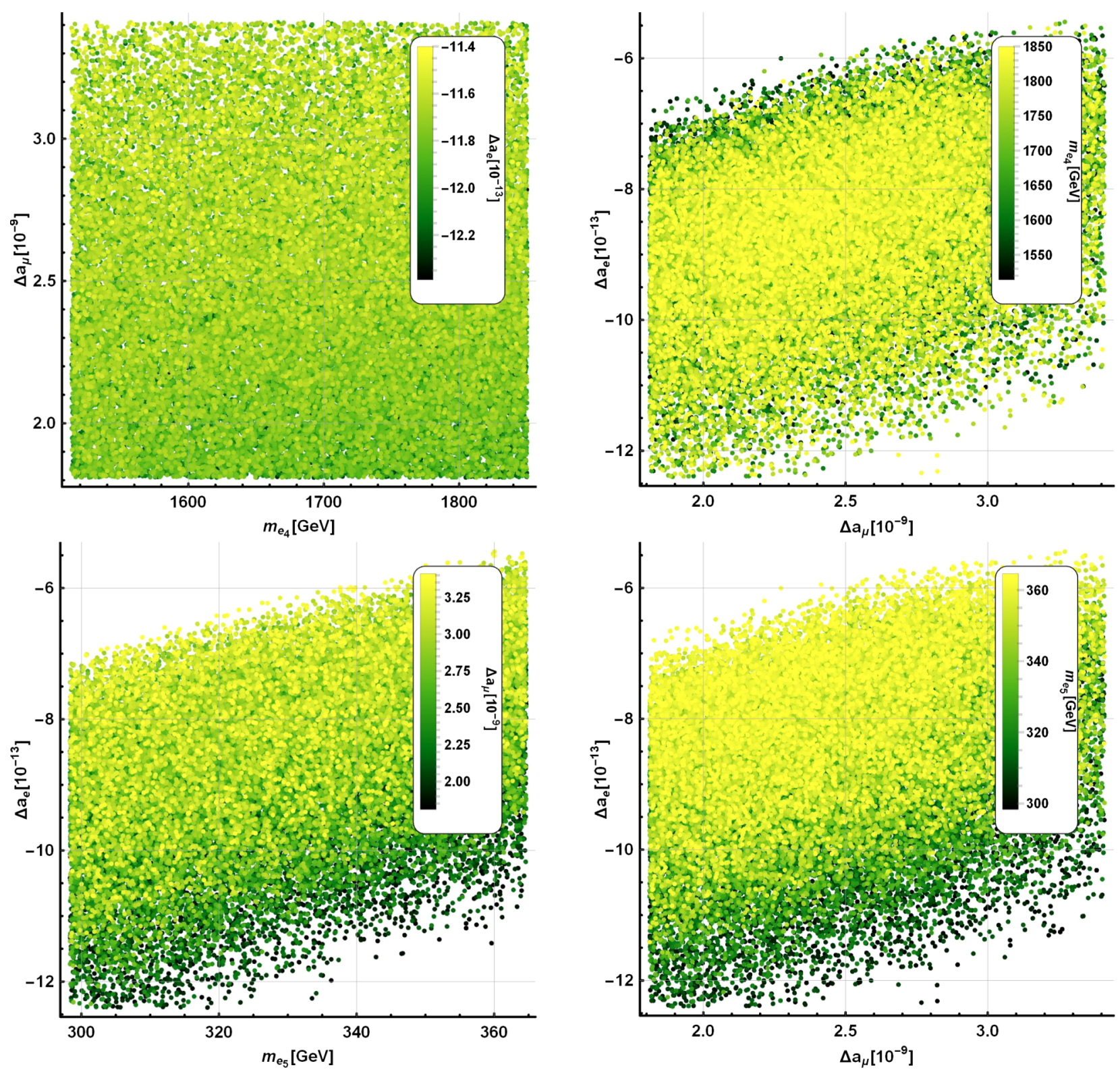

FIG. 12. Available parameter spaces for the muon anomaly(electron anomaly) versus a relevant vectorlike mass $m_{e_{4}}\left(m_{e_{5}}\right)$ with another anomaly(two left plots) in bar where $m_{e_{4}}\left(m_{e_{5}}\right)$ is simplified notation for $M_{44}^{e}\left(M_{55}^{L}\right)$, while the two right plots for the muon anomaly versus electron anomaly with a vectorlike mass $m_{e_{4}}\left(m_{e_{5}}\right)$

how well a benchmark point is converged and by a factor of $\kappa$. However, these plots still tells a correlation between both anomalies and a tendency that the lighter mass of $H_{1}$ is located at edge region of the parameter space. Mass of the lightest non-SM scalar $H_{1}$ implied in top-left plot of Fig. 11 is ranged from 200 to $220 \mathrm{GeV}$ [95] and the cross section for this light non-SM scalar will be compared to that for SM Higgs in appendix. As for mass range of the other non-SM scalars confirmed in rest of other plots in Fig. 11, they all implied heavier mass than that of $H_{1}$ which can be flexible depending on how the parameters are converged as seen in each case of Table IV.
We investigate a correlation for an anomaly versus a relevant mass parameter with another anomaly in bar in Fig. 12. Note that the fourth vectorlike mass is relevant only for the muon anomaly, whereas the fifth is only for the electron anomaly. Even though the fourth (fifth) is irrelevant to the electron (muon) anomaly, it is good to express them together since we rearrange the mass parameters and the anomalies in bar for comparison. The top-left plot in Fig. 12 just fills in whole parameter region, thus no any correlation between the fourth vectorlike mass and the muon anomaly is identified. After we rearranged the order of $m_{e_{4}}$ and $\Delta a_{\mu, e}$ from the top-left 
plot, we can confirm the similar correlation identified in Fig. 11 from the top-right plot in Fig. 12. The bottom-left plot identifies some correlation between the fifth vectorlike mass and the electron anomaly contrary to the top-left plot. For the fifth vectorlike mass, we put the constraint that the lightest vectorlike mass should be greater than $200 \mathrm{GeV}$ [94] and the mass region below $200 \mathrm{GeV}$ is all excluded. After rearranging the order of $m_{e_{5}}$ and $\Delta a_{\mu}$ as in the above plot, we confirmed the similar correlation appears in the bottom-right plot. Interestingly, the topright and the bottom-right plots check the similar correlation.

We confirmed that the muon and electron anomalous magnetic moments with vectorlike particles can be explained to within $1 \sigma$ constraint of each anomaly in a unified way, which is based on two attributes; the first one is the extended scalar sector and the second one is related with the contributions of the vectorlike leptons. The first one which is reflected in our prediction for both anomalies, consists of four non-SM scalars and these contributions play a crucial role for determining the magnitude of each anomaly. The second one is seen by two vertices of both anomaly diagrams. The other Yukawa interactions can take place at each vertex since the vectorlike leptons come in the loop, which is differentiated by the case where the normal SM particles enter in the loop. To be more specific, the helicity flip mass caused by the vectorlike fermions in the $C P$-even and $C P$-odd basis couples the initial particle inside the loop to another particle of different chirality, thus allowing different interactions at each vertex. This means that the different sign problem can be solved by only considering multiplication of the Yukawa constants of each vertex and this property will be covered in detail in next subsection.

\section{Vacuum stability}

An important feature of our extended 2HDM theory is that it predicts large values for the Yukawa coupling constants $y_{2,1}, x_{2,1}$ which can be ideally order of unity in our model. If the Yukawa coupling constants are much lower than unity, which means $y_{2,1}, x_{2,1} \ll 1$, it will not cause any problem for stabilization of the scalar potential. However, large values of the leptonic Yukawa couplings are required in our model to successfully explain both $g-2$ anomalies within the $1 \sigma$ experimentally allowed range and since they are somehow related with the electroweak sector parameters, it might be able to destabilize the Higgs potential. As previously mentioned, in our analysis of the scalar sector and $g-2$ anomalies we are restricting to the scenario of decoupling limit, which implies that the large values of the leptonic Yukawa couplings will have a very small impact in the stability of up-type Higgs $H_{u}$ potential, whereas the conditons for the stability of the down type Higgs $H_{d}$ potential need to be determined. To discuss the stability of the scalar potential, one has to analyze its quartic terms because they will dominate the behavior of the scalar potential in the region of very large values of the field components. To this end, the quartic terms of the scalar potential are written in terms of the Hermitian bilinear combination of the scalar fields. To simplify our analysis, we discuss the stability conditions of the resulting $2 \mathrm{HDM}$ scalar potential arising after the gauge singlet scalar field $\phi$ acquires a vacuum expectation value. Such stability conditions have been analyzed in detail in the framework of $2 \mathrm{HDM}$ in $[96,97]$. In order to analyze the stability of the $H_{d}$ potential, what we need to check if the quartic scalar couplings in each case of Table III fulfill the stability conditions to be determined below. Given that our Higgs potential corresponds to the one of an extended $2 \mathrm{HDM}$ with the flavon field $\phi$, in order to apply the stability conditions used in the Ref. [96] to our Higgs potential, we need to reduce the number of scalar degrees of freedom by considering the resulting 2HDM scalar potential arising after the gauge singlet scalar field $\phi$ is integrated out. From the scalar potential it follows that the relevant quartic coupling constant $\lambda_{6}$ must be positive, otherwise the vev $v_{3}$ would fall into negative infinity when the field $\phi$ value increases. For the same reason, the quartic coupling constants $\lambda_{1,2}$ must also be positive. From the aforementioned stability conditions we conclude that the cases A and $\mathrm{C}$ must be excluded since their corresponding quartic coupling constants $\lambda_{2}$ are negative. Assuming the flavon field $\phi$ develops its vev $v_{3}$, we can rewrite the Higgs potential in terms of $H_{u}$ and $H_{d}$ fields as follows:

$$
\begin{aligned}
V= & \mu_{1}^{2}\left(H_{u} H_{u}^{\dagger}\right)+\mu_{2}^{2}\left(H_{d} H_{d}^{\dagger}\right)+\mu_{3}^{2} \frac{v_{3}^{2}}{2}+2 \mu_{s b}^{2} \frac{v_{3}^{2}}{2} \\
& +\lambda_{1}\left(H_{u} H_{u}^{\dagger}\right)^{2}+\lambda_{2}\left(H_{d} H_{d}^{\dagger}\right)^{2} \\
& +\lambda_{3}\left(H_{u} H_{u}^{\dagger}\right)\left(H_{d} H_{d}^{\dagger}\right)+\lambda_{4}\left(H_{u} H_{d}^{\dagger}\right)\left(H_{d} H_{u}^{\dagger}\right) \\
& +\lambda_{5}\left(\varepsilon_{i j} H_{u}^{i} H_{d}^{j} \frac{v_{3}^{2}}{2}+H . c\right) \\
& +\lambda_{6} \frac{v_{3}^{4}}{4}+\lambda_{7} \frac{v_{3}^{2}}{2}\left(H_{u} H_{u}^{\dagger}\right)+\lambda_{8} \frac{v_{3}^{2}}{2}\left(H_{d} H_{d}^{\dagger}\right) .
\end{aligned}
$$

Dropping all numbers and combining same order terms, the Higgs potential becomes much simpler as follows:

$$
\begin{aligned}
V= & \left(\mu_{1}^{2}+\lambda_{7} \frac{v_{3}^{2}}{2}\right)\left(H_{u} H_{u}^{\dagger}\right)+\left(\mu_{2}^{2}+\lambda_{8} \frac{v_{3}^{2}}{2}\right)\left(H_{d} H_{d}^{\dagger}\right) \\
& +\lambda_{1}\left(H_{u} H_{u}^{\dagger}\right)^{2}+\lambda_{2}\left(H_{d} H_{d}^{\dagger}\right)^{2} \\
& +\lambda_{3}\left(H_{u} H_{u}^{\dagger}\right)\left(H_{d} H_{d}^{\dagger}\right)+\lambda_{5} \frac{v_{3}^{2}}{2}\left(\varepsilon_{i j} H_{u}^{i} H_{d}^{j}+H . c\right)
\end{aligned}
$$

where it is worth mentioning that the $\lambda_{4}$ term can be safely removed in the Higgs potential since it does not play a role in the $C P$-even, odd but charged mass matrix (Now our focus is the neutral scalar sectors). Here, we can impose one 
extra condition for the stabilization check, which is that the redefined mass terms must be negative, otherwise we get zero vev as a global minimum.

$$
\begin{aligned}
\mu_{1}^{2}+\lambda_{7} \frac{v_{3}^{2}}{2} & =-2 \lambda_{1} v_{1}^{2}-\lambda_{3} v_{2}^{2}+2 \lambda_{3} v_{2}^{2}=-2 \lambda_{1} v_{1}^{2}+\lambda_{3} v_{2}^{2}<0 \\
\mu_{2}^{2}+\lambda_{8} \frac{v_{3}^{2}}{2} & =-2 \lambda_{2} v_{2}^{2}+\lambda_{3} v_{1}^{2}-\frac{1}{2} \lambda_{8} v_{3}^{2}+\lambda_{8} \frac{v_{3}^{2}}{2} \\
& =-2 \lambda_{2} v_{2}^{2}+\lambda_{3} v_{1}^{2}<0
\end{aligned}
$$

We have used the decoupling limit of Eq. (58) at the first equality of Eq. (79). From this equation, it is possible to determine the appropriate sign for the quartic coupling constant $\lambda_{3}$. In our numerical analysis, the vev $v_{1}$ is much dominant than the vev $v_{2}$ so it leads to a negative sign for the quartic coupling constant $\lambda_{3}$, otherwise the below equation of Eq. (79) would become positive. The sign of the quartic coupling constant $\lambda_{3}$ also determines the one of $\lambda_{5,7}$ in the decoupling scenario, which means that $\lambda_{5,7}$ must also be negative. On top of that, the large Yukawa coupling constants $y, x$ can be understood in connection with the vev $v_{3}$. To this end, we consider the definition for the Yukawa coupling constants $x_{1}$ and $x_{2}$, which are given by:

$$
x_{2}=\left|\frac{y_{\mu} M_{44}}{y_{2} v_{3}}\right|, \quad x_{1}=\left|\frac{y_{e} M_{55}}{y_{1} v_{3}}\right|,
$$

where in order to successfully explain both $g-2$ anomalies within the $1 \sigma$ experimentally allowed range, one has to rely on small values of $v_{3}$, which are $\mathcal{O}(10 \mathrm{GeV})$, and the small values of $v_{3}$ do not significantly spoil the down-type Higgs $H_{d}$ potential as seen in Eq. (79). In other words, the mass parameters $\mu_{1,2}^{2}$ are much larger than the parameters $\lambda_{7,8} v_{3}^{2} / 2$, thus allowing more freedom in the sign of $\lambda_{8}$. Then, we are now ready to match our simplified Higgs potential with the one given in the Ref. [96]. Taking into consideration that our Higgs alignment is different than the one of [96], our mass parameters can be redefined as follows:

$m_{11}^{2}=\mu_{1}^{2}+\lambda_{7} \frac{v_{3}^{2}}{2}, \quad m_{22}^{2}=\mu_{2}^{2}+\lambda_{8} \frac{v_{3}^{2}}{2}, \quad m_{12}^{2}=\lambda_{5} \frac{v_{3}^{2}}{2}$

$\beta_{1}=2 \lambda_{1}, \quad \beta_{2}=2 \lambda_{2}, \quad \beta_{3}=\lambda_{3}, \quad \beta_{4}=0, \quad \beta_{5}=0$.

Then, following [96,97], it is found that the scalar potential is stable, when the following relations are fulfilled:

$$
\begin{array}{r}
\beta_{1} \geq 0, \quad \beta_{2} \geq 0, \quad \beta_{3}+\sqrt{\beta_{1} \beta_{2}} \geq 0 \\
\beta_{3}+\beta_{4}+\sqrt{\beta_{1} \beta_{2}}>\left|\beta_{5}\right| \rightarrow \beta_{3}+\sqrt{\beta_{1} \beta_{2}}>0 .
\end{array}
$$

The last stability condition can be rewritten as shown on the right side since the $\beta_{4,5}$ are zero in our Higgs potential and the cases B and D must be excluded by this last condition shown in Eq. (84). The conditions given in Eqs. (83) and (84) are crucial to guarantee the stability of the electroweak vacuum. Furthermore, one has to require that the squared masses for the physical scalars are positive. Besides that, according to [96], the minimum of the scalar potential is a global minimum when the following condition is fulfilled:

$$
\begin{aligned}
& m_{12}^{2}\left(m_{11}^{2}-m_{22}^{2} \sqrt{\frac{\beta_{1}}{\beta_{2}}}\right)\left(\tan \beta-\sqrt{4} \frac{\beta_{1}}{\beta_{2}}\right)>0 \\
& \rightarrow m_{12}^{2}\left(m_{11}^{2}-m_{22}^{2} \sqrt{\frac{\beta_{1}}{\beta_{2}}}\right)>0
\end{aligned}
$$

where the latter condition on the left-hand side is always successfully fulfilled for all cases, so we can simply drop off the condition as shown on the right side. Then, it is enough to confirm whether each case satisfies the reduced global minimum condition and the case E successfully fulfills that requirement as shown below:

$$
\begin{aligned}
& m_{12}^{2}=-1763.9 \mathrm{GeV}^{2}, \quad m_{11}^{2}=-7896.5 \mathrm{GeV}^{2}, \\
& m_{22}^{2}=-43258.8 \mathrm{GeV}^{2}, \quad \sqrt{\frac{\beta_{1}}{\beta_{2}}}=0.0791994 \\
& m_{12}^{2}\left(m_{11}^{2}-m_{22}^{2} \sqrt{\frac{\beta_{1}}{\beta_{2}}}\right) \approx 7.886 \times 10^{6} \mathrm{GeV}^{4}>0 .
\end{aligned}
$$

Thus, we have numerically checked that the best fit point corresponding to the case E obtained in the numerical analysis of the scalar potential and $g-2$ muon and electron anomalies is consistent with the above given stability conditions of the scalar potential and at the same time ensure positive values for the squared masses of the physical scalars, consistent with the current experimental data. Finally, to close this section, it is worth mentioning that the large Yukawa coupling constants $y$, $x$ involve the small vev $v_{3}$ in our model and this ensures that not only the $H_{u}$ potential is stable in the decoupling scenario but also the $H_{d}$ potential successfully fulfill the requirements of vacuum stability for both the small vev $v_{3}$ and appropriate values of the quartic scalar couplings.

\section{How is the scalar exchange possible to accommodate both anomalies at $1 \sigma$ constraint analytically?}

In order to analyze how the scalar exchange is able to explain both anomalies within the $1 \sigma$ range, we revisit the analytic expressions for both muon and electron anomalous magnetic moments: 


$$
\begin{aligned}
\Delta a_{\mu}= & y_{24}^{e} x_{42}^{e} \frac{m_{\mu}^{2}}{8 \pi^{2}}\left[\left(R_{e}^{T}\right)_{22}\left(R_{e}^{T}\right)_{32} I_{S}^{(\mu)}\left(m_{e_{4}}, m_{H_{1}}\right)+\left(R_{e}^{T}\right)_{23}\left(R_{e}^{T}\right)_{33} I_{S}^{(\mu)}\left(m_{e_{4}}, m_{H_{2}}\right)\right. \\
& \left.-\left(R_{o}^{T}\right)_{22}\left(R_{o}^{T}\right)_{32} I_{P}^{(\mu)}\left(m_{e_{4}}, m_{A_{1}}\right)-\left(R_{o}^{T}\right)_{23}\left(R_{o}^{T}\right)_{33} I_{P}^{(\mu)}\left(m_{e_{4}}, m_{A_{2}}\right)\right], \\
\Delta a_{e}= & y_{51}^{e} x_{15}^{L} \frac{m_{e}^{2}}{8 \pi^{2}}\left[\left(R_{e}^{T}\right)_{22}\left(R_{e}^{T}\right)_{32} I_{S}^{(e)}\left(m_{e_{5}}, m_{H_{1}}\right)+\left(R_{e}^{T}\right)_{23}\left(R_{e}^{T}\right)_{33} I_{S}^{(e)}\left(m_{e_{5}}, m_{H_{2}}\right)\right. \\
& \left.-\left(R_{o}^{T}\right)_{22}\left(R_{o}^{T}\right)_{32} I_{P}^{(e)}\left(m_{e_{5}}, m_{A_{1}}\right)-\left(R_{o}^{T}\right)_{23}\left(R_{e}^{T}\right)_{33} I_{P}^{(E)}\left(m_{e_{5}}, m_{A_{2}}\right)\right],
\end{aligned}
$$

where

$$
I_{S(P)}^{(e, \mu)}\left(m_{E_{4,5},}, m_{S}\right)=\int_{0}^{1} \frac{x^{2}\left(1-x \pm \frac{m_{E_{4,5}}}{m_{e, \mu}}\right)}{m_{e, \mu}^{2} x^{2}+\left(m_{E_{4,5}}^{2}-m_{e, \mu}^{2}\right) x+m_{S, P}^{2}(1-x)} d x
$$

with $S(P)$ corresponding to scalar (pseudoscalar) and $E_{4,5}$ standing for the vectorlike family. Furthermore, $E_{4}$ and $E_{5}$ only contribute to the muon and electron anomalous magnetic moments, respectively.

First of all, we focus on the sign of each anomaly. The different signs of each anomaly indicated by the $1 \sigma$ experimentally allowed range can be understood at the level of Yukawa constants apart from the loop structures. As seen in Table V, the Yukawa coefficient $y$ can be either positive or negative, while $x$ only remains positive since we take the absolute value to the $x$. We also considered the case

TABLE V. Initial parameter setup.

\begin{tabular}{lc}
\hline \hline Parameter & Value/Scanned Region $(\mathrm{GeV})$ \\
\hline$v_{u}=v_{1}$ & $\frac{\tan \beta}{\sqrt{1+\tan \beta^{2}}} \times 246$ \\
$v_{d}=v_{2}$ & $\frac{1}{\sqrt{1+\tan \beta^{2}}} \times 246$ \\
$v_{\phi}=v_{3}$ & $\pm[0.01,1.00] \times 1000$ \\
$\tan \beta=v_{u} / v_{d}$ & {$[5,50]$} \\
$\lambda_{1}$ & $\left(m_{h}^{2}-\frac{v_{2} v_{2}^{2} \lambda_{5}}{2 v_{1}}\right) /\left(4 v_{1}^{2}\right)$ \\
$\lambda_{2}$ & $\pm[0.50,12.00]$ \\
$\lambda_{3}$ & $\pm[0.50,12.00]$ \\
$\lambda_{4}$ & $\pm[0.50,12.00]$ \\
$\lambda_{5}$ & $4 v_{1} v_{2} \lambda_{3} /\left(v_{3}\right)^{2}$ \\
$\lambda_{6}$ & $\pm[0.50,12.00]$ \\
$\lambda_{7}$ & $v_{2} \lambda_{5} / v_{1}$ \\
$\lambda_{8}$ & $\pm[0.50,12.00]$ \\
$M_{44}^{e}$ & {$\left[2 \times 10^{2}, 2 \times 10^{3}\right]$} \\
$M_{55}^{L}$ & {$\left[2 \times 10^{2}, 2 \times 10^{3}\right]$} \\
$\mu_{s b}$ & $i[0,1] \times[300,500]$ \\
$y_{e}$ & $\sqrt{2} m_{e} / v_{2}$ \\
$y_{\mu}$ & $\sqrt{2} m_{\mu} / v_{2}$ \\
$y_{24}^{e}=y_{2}$ & $\pm[1.0,3.5]$ \\
$y_{51}^{e}=y_{1}$ & $\pm[1.0,3.5]$ \\
$x_{42}^{e}=x_{2}$ & $\left|y_{\mu} M_{44}^{e} /\left(y_{24}^{e} v_{3}\right)\right|$ \\
$x_{15}^{L}=x_{1}$ & $\left|y_{e} M_{55}^{L} /\left(y_{51}^{e} v_{3}\right)\right|$ \\
\hline \hline &
\end{tabular}

where the coefficients $x, y$ are purely positive, assuming $v_{3}$ is positive, without taking absolute value and the multiplication of the Yukawa coefficients $x \times y$ cannot change the sign of each anomaly since the denominator of $x$ includes $y$ and they are cancel out. Then, the sign problem depends on summing over loop functions and we found that the order of the muon anomaly prediction is suitable, whereas the corresponding to the electron anomaly is about $10^{-16}$ which is too small to be accommodated within the $1 \sigma$ experimentally allowed range. Therefore, we found that taking an absolute value to one of the Yukawa coefficients is an appropriate strategy for the sign and allows to reproduce the correct order of magnitude of each anomaly allowed by the $1 \sigma$ experimentally allowed range, for an appropriate choice of the model parameters. This feature is a crucial difference compared with the $W$ or $Z^{\prime}$ gauge boson exchange [33]. The $W$ gauge boson exchange covered in the main body of this work keeps the same coupling constant at each vertex, therefore it is completely different from the scalar exchange with vectorlike leptons. For the $Z^{\prime}$ exchange covered in [33], it has the common property that the coupling constant of each vertex is different to each other, whereas the coupling constants of the $Z^{\prime}$ are more constrained by the mixing angle between $i$ th chiral family and fourth vectorlike family, so it is impossible to explain both anomalies at the same time. As a result, allowing different Yukawa constants with appropriate signs enables both anomalies to be explained in a unified way.

Next we turn our attention to the order of magnitude of our predictions for both anomalies. Considering that the sign problem is solved by having each Yukawa constant $y$ either positive or negative, it can be easily understood that inside the structure in parentheses of Eq. (88) should imply the same direction, which is is determined by the contribution of all loop functions in parentheses. Since the mass difference among non-SM scalars and vectorlike particles is not so big, we have to consider their masses in the computation of muon and electron anomalous 
magnetic moments, as follows from Eq. (88). For an easy analysis, we take the case E reported in Table III and suppose that

$$
\begin{aligned}
&\left(R_{e}^{T}\right)_{22}\left(R_{e}^{T}\right)_{32}=c_{1},\left(R_{e}^{T}\right)_{23}\left(R_{e}^{T}\right)_{33}=-c_{1}, \\
&\left(R_{o}^{T}\right)_{22}\left(R_{o}^{T}\right)_{32}=c_{2},\left(R_{o}^{T}\right)_{23}\left(R_{o}^{T}\right)_{33}=-c_{2}, \\
& I_{S}^{\mu}\left(m_{e_{4}}, m_{H_{1}}\right)=d_{1}, I_{S}^{\mu}\left(m_{e_{4}}, m_{H_{2}}\right)=d_{2}, \\
& I_{P}^{\mu}\left(m_{e_{4}}, m_{A_{1}}\right)=-d_{3}, I_{P}^{\mu}\left(m_{e_{4}}, m_{A_{2}}\right)=-d_{4}, \\
& I_{S}^{e}\left(m_{e_{5}}, m_{H_{1}}\right)=e_{1}, I_{S}^{e}\left(m_{e_{5}}, m_{H_{2}}\right)=e_{2}, \\
& I_{P}^{e}\left(m_{e_{5}}, m_{A_{1}}\right)=-e_{3}, I_{P}^{e}\left(m_{e_{5}}, m_{A_{2}}\right)=-e_{4}, \\
& d_{1}>d_{3}>d_{2}>d_{4}, \quad e_{1}>e_{3}>e_{2}>e_{4}
\end{aligned}
$$

where $c_{1,2}$ are arbitrary constant between 0 and 1 either positive or negative and mass ordering among $d(e)_{i},(i=1,2,3,4)$ can be easily understood by considering mass difference between non-SM scalars and vectorlike particles. The muon and electron anomaly prediction can be rewritten in terms of these redefined constants:

$$
\begin{aligned}
\Delta a_{\mu} & =y_{2} x_{2} \frac{m_{\mu}^{2}}{8 \pi^{2}}\left[c_{1} d_{1}-c_{1} d_{2}+c_{2} d_{3}-c_{2} d_{4}\right] \\
& =y_{2} x_{2} \frac{m_{\mu}^{2}}{8 \pi^{2}}\left[c_{1}\left(d_{1}-d_{2}\right)+c_{2}\left(d_{3}-d_{4}\right)\right] \\
& =y_{2} x_{2} \frac{m_{\mu}^{2}}{8 \pi^{2}}\left[c_{1} d_{12}+c_{2} d_{34}\right] \\
\Delta a_{e} & =y_{1} x_{1} \frac{m_{e}^{2}}{8 \pi^{2}}\left[c_{1} e_{1}-c_{1} e_{2}+c_{2} e_{3}-c_{2} e_{4}\right] \\
& =y_{1} x_{1} \frac{m_{e}^{2}}{8 \pi^{2}}\left[c_{1}\left(e_{1}-e_{2}\right)+c_{2}\left(e_{3}-e_{4}\right)\right] \\
& =y_{1} x_{1} \frac{m_{e}^{2}}{8 \pi^{2}}\left[c_{1} e_{12}+c_{2} e_{34}\right]
\end{aligned}
$$

where $y_{2}, x_{2}, y_{1}, x_{1}$ are simplified notation for $y_{24}^{e}, x_{42}^{e}, y_{51}^{e}, x_{15}^{L}, \quad$ respectively, and $d(e)_{i j} \equiv d(e)_{i}-$ $d(e)_{j}$ and $d(e)_{i j}$ are positive. Since the inside structure in parentheses depends on relative magnitude of both $c_{1,2}$ and $d(e)_{i j}$ at this stage where no more analytic simplification is possible, it is good to implement a specific value for them. Referring the values used to derive the result of case E, they are

$$
\begin{aligned}
y_{2} x_{2} \frac{m_{\mu}^{2}}{8 \pi^{2}} c_{1} d_{1} & =-4.629 \times 10^{-7}, \\
y_{1} x_{1} \frac{m_{e}^{2}}{8 \pi^{2}} c_{1} e_{1} & =-8.532 \times 10^{-12} \\
-y_{2} x_{2} \frac{m_{\mu}^{2}}{8 \pi^{2}} c_{1} d_{2} & =4.520 \times 10^{-7}, \\
-y_{1} x_{1} \frac{m_{e}^{2}}{8 \pi^{2}} c_{1} e_{2} & =6.808 \times 10^{-12} \\
y_{2} x_{2} \frac{m_{\mu}^{2}}{8 \pi^{2}} c_{2} d_{3} & =7.984 \times 10^{-8}, \\
y_{1} x_{1} \frac{m_{e}^{2}}{8 \pi^{2}} c_{2} e_{3} & =1.323 \times 10^{-12} \\
-y_{2} x_{2} \frac{m_{\mu}^{2}}{8 \pi^{2}} c_{2} d_{4} & =-6.659 \times 10^{-8}, \\
-y_{1} x_{1} \frac{m_{e}^{2}}{8 \pi^{2}} c_{2} e_{4} & =-5.217 \times 10^{-13}
\end{aligned}
$$

and summing over all values in left or right column of Eq. (92) yields the prediction for muon and electron anomaly at $1 \sigma$

$$
\begin{gathered}
\Delta a_{\mu}=y_{2} x_{2} \frac{m_{\mu}^{2}}{8 \pi^{2}}\left[c_{1} d_{1}-c_{1} d_{2}+c_{2} d_{3}-c_{2} d_{4}\right]=2.393 \times 10^{-9} \\
\Delta a_{e}=y_{1} x_{1} \frac{m_{e}^{2}}{8 \pi^{2}}\left[c_{1} e_{1}-c_{1} e_{2}+c_{2} e_{3}-c_{2} e_{4}\right]=-9.232 \times 10^{-13} .
\end{gathered}
$$

\section{CONCLUSION}

We have proposed a model to account for the hierarchical structure of the SM Yukawa couplings. In our approach the SM is an effective theory arising from a theory with extended particle spectrum and symmetries. The considered model includes an extension of the 2HDM where the particle spectrum is enlarged by the inclusion of two vectorlike fermion families, right-handed Majorana neutrinos and a gauge singlet scalar field, together with the inclusion of a global $U(1)^{\prime}$ symmetry spontaneously broken at the TeV scale. Since the $U(1)^{\prime}$ symmetry is global, this model does not feature a $Z^{\prime}$ boson and it is softly broken in the 2HDM potential to avoid a Goldstone boson. Its main effect is to forbid SM Yukawa interactions due to the $U(1)^{\prime}$ charge conservation. Besides that, this model has the property of the 2HDM type II where one Higgs doublet couples with the up-type fermions whereas the remaining one has Yukawa interactions with downtype fermions, where such couplings are allowed between chiral fermions and vectorlike fermions due to the choice of $U(1)^{\prime}$ charges (chiral fermions having zero charges while vectorlike fermions, Higgs and flavons have charges \pm 1 ). Below the mass scale of the vectorlike fermions, such 
couplings result in effective Yukawa couplings suppressed by a factor $\langle\phi\rangle / M$ where the numerator is the vev of the flavon and the denominator is the vectorlike mass. This factor naturally determines the magnitude of SM interactions and the mass scale for the vectorlike fermions under a suitable choice of the flavon vev. We have developed a mixing formalism based on $7 \times 7$ mass matrices to describe the mixing of the three chiral families with the two vectorlike families.

Within the above proposed model, we have focused on accommodating the long-established muon and less established electron anomalous magnetic moments at one-loop level. A main difficulty arises from the sign of each anomalous deviation of the experimental value from its SM prediction. Generally, the Feynman diagrams for the muon and electron anomalous magnetic moments have the same structure except from the fact that the external particles are different, which makes it difficult to flip the sign of each contribution. Specifically we have required that both deviations in Eq. (1) at oneloop should be accommodated within the $1 \sigma$ experimentally allowed range, which is a challenging requirement.

We first considered in detail the $W$ boson exchange contributions to the muon and electron anomalous magnetic moments at one-loop. The relevant sector for the $W$ boson exchange is that of the neutrino and we analyzed a novel operator that generates the masses of the light active neutrinos in this model. The well-known five dimensional Weinberg operator which we refer as type Ia seesaw mechanism does not work in this model since it is forbidden by the $U(1)$ symmetry due to the fact that both $S U(2)$ scalar doublets are negatively charged under this symmetry. For this reason, we made use of the Weinberg-like operator known as type Ib seesaw mechanism allowed in this model. With the type $\mathrm{Ib}$ seesaw mechanism, we built the neutrino mass matrix with two vectorlike neutrinos and ignored fifth vectorlike neutrinos since they are too heavy to contribute to the phenomenology. The deviation of unitarity $\eta$ derived from the heavy vectorlike neutrinos plays a crucial role for enhancing the sensitivity of the CLFV $\mu \rightarrow e \gamma$ decay to the observable level. Furthermore, the Yukawa constants of Dirac neutrino mass matrix can be connected to the observables measured in neutrino oscillation experiments. One of the neutrino Yukawa constants is defined with a suppression factor $\epsilon$. Therefore, the effective $3 \times 3$ neutrino mass matrix tells that the tiny masses of the light active neutrinos depend on the mass scale of vectorlike neutrinos as well as on the suppression factor $\epsilon$. This implies that mass scale of vectorlike neutrinos is not required to be of the order of $10^{14} \mathrm{GeV}$, as in the conventional type Ia seesaw mechanism. In our proposed model, the vectorlike neutrinos can have masses at the $\mathrm{TeV}$ scale, thus allowing to test our model at colliders. Those vectorlike neutrinos can be pair produced at the LHC via Drell-Yan annihilation mediated by a virtual $Z$ gauge boson. They can also be produced in association with a SM charged lepton via Drell-Yan annihilation mediated by a $W$ gauge boson. These heavy vector like sterile neutrinos can decay into a SM charged lepton and light active neutrinos. Thus, the heavy neutrino pair production at a proton-proton collider will give rise to an opposite sign dilepton final state, which implies that the observation of an excess of events in this final state over the SM background can be a smoking gun signature of this model, whose observation will be crucial to assess its viability. It is confirmed that the branching ratio of $\mu \rightarrow e \gamma$ decay can be expressed in terms of the deviation of unitarity $\eta$ as shown in [11,82] and our prediction for the muon and electron anomalous magnetic moments can also be written in terms of nonunitarity. We derived the analytic expression for the anomalies and found that the order of magnitude of these predictions is too small to accommodate the experimental bound within the $1 \sigma$ range and the sign of each prediction also points out in the same direction. Therefore, we concluded that the $W$ boson exchange at one-loop is not enough to explain both anomalies at $1 \sigma$ and this conclusion has been a good motivation to search for another possibility such as scalar exchange, which is one of the main purposes of this work.

We then turned our attention to the 2HDM contributions (inclusion also of the singlet scalar $\phi$ ) to the muon and electron anomalous magnetic moments, assuming by a choice of parameters a diagonal charged lepton mass matrix to suppress the branching ratio of $\mu \rightarrow e \gamma$. In our analysis we considered in detail the scalar sector of our model, which is composed of two $S U(2)$ scalar doublets $H_{u}$ and $H_{d}$ and one electrically neutral complex scalar $\phi$ by studying the corresponding scalar potential, deriving the squared mass matrices for the $C P$-even, $C P$-odd neutral and electrically charged scalars and determining the resulting scalar mass spectrum. We have restricted to the scenario corresponding to the decoupling limit where no mixing between the physical SM Higgs $h$ and the physical non-SM scalars $H_{1,2}$ arise and within this scenario we have imposed the restrictions arising from the Higgs diphoton decay rate, the $h W W$ coupling, the $125 \mathrm{GeV}$ mass of the SM-like Higgs and the experimental lower bounds on non-SM scalar masses, to determine the allowed parameter space 
consistent with the muon and electron anomalous magnetic moments. To this end, we have constructed a $\chi^{2}$ fitting function, which measures the deviation of the values of the physical observables obtained in the model, i.e., $(g-2)_{e, \mu}$, the $125 \mathrm{GeV}$ SM-like Higgs mass, the Higgs diphoton signal strength, the $h W W$ coupling, with respect to their experimental values. Its minimization allows to determine the values of the model parameters consistent with the measured experimental values of these observables. After saturating the $\chi^{2}$ value less than or nearly 2 , we obtained five independent benchmark points and carried out second scan with the benchmark points to find a correlation between observables and mass parameters. For the plots, we took an appropriate case which is more converged when compared to other ones and satisfying the vacuum stability conditions. We found that our prediction for both anomalies can be explained within the $1 \sigma$ constraint of each anomaly and a correlation proportional for muon versus electron anomaly is appeared in Fig. 11 and 12. Here, we put two constraints on mass of the lightest non-SM scalar and of the lightest vectorlike family; $m_{H_{1}}, m_{e_{5}}>200 \mathrm{GeV}$ based on references. The second scan result tells that the available parameter space is not significantly constrained by current experimental results on non-SM scalar mass and vectorlike mass, while keeping perturbativity for quartic couplings and Yukawa constants. An important feature of our BSM model is it predicts the large Yukawa coupling constants $y$, $x$, which might be able to destabilize the Higgs potential. The up-type Higgs $H_{u}$ potential is not significantly affected by the large Yukawa coupling constants in the decoupling scenario, whereas there is no safe condition for the downtype Higgs $H_{d}$ potential which can be worsen by mixing with the flavon field $\phi$. The large Yukawa coupling constants $x$ introduces small values for the vev $v_{3}$ in the definition of $x$ and the energy scale is confirmed by order of $10 \mathrm{GeV}$ in our numerical analysis. On top of that, we also identified the appropriate sign of quartic coupling constants can make the Higgs potential stable. Therefore, the down type $H_{d}$ Higgs potential is stable by both the small vev $v_{3}$ and the appropriate quartic coupling constants in our BSM model. Lastly, we discussed how we were able to explain both $(g-2)_{e, \mu}$ anomalies at $1 \sigma$ constraint and impact of the light non-SM scalar $H_{1}$. For the former, we first simplified the prediction for both anomalies and used some numerical values at the stage where no more analytic simplification is possible. For the latter, we compared the cross section for the SM process $p p \rightarrow h$ and BSM process $p p \rightarrow H_{1}$ and included this comparison in Appendix B.

We conclude that the proposed model of fermion mass hierarchies is able to successfully accommodate both the muon and electron anomalous magnetic moments within the $1 \sigma$ experimentally allowed ranges, with the dominant contributions arising from one loop diagrams involving the 2HDM scalars and vectorlike leptons. The resulting model parameter space consistent with the $(g-2)_{e, \mu}$ anomalies requires masses of non-SM scalars and vectorlike particles in the sub $\mathrm{TeV}$ and $\mathrm{TeV}$ ranges, thus making these particles accessible at the LHC and future colliders.

\section{ACKNOWLEDGMENTS}

We would like to thank Simon King for discussions. This research has received funding from ANID-Chile FONDECYT 1210378. S. F. K. acknowledges the STFC Consolidated Grant No. ST/L000296/1 and the European Union's Horizon 2020 Research and Innovation programme under Marie Skłodowska-Curie grant agreements Elusives ITN No. 674896, HIDDeN European ITN project (H2020-MSCA-ITN-2019//860881-HIDDeN) and InvisiblesPlus RISE No. 690575.

\section{APPENDIX A: QUARK MASS MATRICES IN TWO BASES}

As the lepton mass matrix is constructed in main body of this work, the quark sector can be built in a similar way. Like the lepton sector, we make use of two approaches to an effective lepton mass matrix, one of which is a convenient basis and the other is a decoupling basis.

\section{A convenient basis for quarks}

Consider the $7 \times 7$ quark mass matrix rotated as in the lepton sector. 


$M^{u}=\left(\begin{array}{c|ccccccc} & u_{1 R} & u_{2 R} & u_{3 R} & u_{4 R} & u_{5 R} & \tilde{Q}_{4 R} & \tilde{Q}_{5 R} \\ \bar{Q}_{1 L} & 0 & 0 & 0 & 0 & y_{15}^{u} v_{u} & 0 & x_{15}^{Q} v_{\phi} \\ \bar{Q}_{2 L} & 0 & 0 & 0 & y_{24}^{u} v_{u} & y_{25}^{u} v_{u} & 0 & x_{25}^{Q} v_{\phi} \\ \bar{Q}_{3 L} & 0 & 0 & 0 & y_{34}^{u} v_{u} & y_{35}^{u} v_{u} & x_{34}^{Q} v_{\phi} & x_{35}^{Q} v_{\phi} \\ \bar{Q}_{4 L} & 0 & 0 & y_{43}^{u} v_{u} & 0 & 0 & M_{44}^{Q} & M_{45}^{Q} \\ \bar{Q}_{5 L} & y_{51}^{u} v_{u} & y_{52}^{u} v_{u} & y_{53}^{u} v_{u} & 0 & 0 & 0 & M_{55}^{Q} \\ \overline{\tilde{u}}_{4 L} & 0 & x_{42}^{u} v_{\phi} & x_{43}^{u} v_{\phi} & M_{44}^{u} & 0 & 0 & 0 \\ \tilde{\tilde{u}}_{5 L} & x_{51}^{u} v_{\phi} & x_{52}^{u} v_{\phi} & x_{53}^{u} v_{\phi} & M_{54}^{u} & M_{55}^{u} & 0 & 0\end{array}\right)$

$$
M^{d}=\left(\begin{array}{c|ccccccc} 
& d_{1 R} & d_{2 R} & d_{3 R} & d_{4 R} & d_{5 R} & \tilde{Q}_{4 R} & \tilde{Q}_{5 R} \\
\hline \bar{Q}_{1 L} & 0 & 0 & 0 & y_{14}^{d} v_{d} & y_{15}^{d} v_{d} & 0 & x_{15}^{Q} v_{\phi} \\
\bar{Q}_{2 L} & 0 & 0 & 0 & y_{24}^{d} v_{d} & y_{25}^{d} v_{d} & 0 & x_{25}^{Q} v_{\phi} \\
\bar{Q}_{3 L} & 0 & 0 & 0 & y_{34}^{d} v_{d} & y_{35}^{d} v_{d} & x_{34}^{Q} v_{\phi} & x_{35}^{Q} v_{\phi} \\
\bar{Q}_{4 L} & 0 & 0 & y_{43}^{d} v_{d} & 0 & 0 & M_{44}^{Q} & M_{45}^{Q} \\
\bar{Q}_{5 L} & y_{51}^{d} v_{d} & y_{52}^{d} v_{d} & y_{53}^{d} v_{d} & 0 & 0 & 0 & M_{55}^{Q} \\
\tilde{\tilde{d}}_{4 L} & 0 & x_{42}^{d} v_{\phi} & x_{43}^{d} v_{\phi} & M_{44}^{d} & 0 & 0 & 0 \\
\overline{\tilde{d}}_{5 L} & x_{51}^{d} v_{\phi} & x_{52}^{d} v_{\phi} & x_{53}^{d} v_{\phi} & M_{54}^{d} & M_{55}^{d} & 0 & 0
\end{array}\right)
$$

Notice that the same rotations operated in the lepton sector is applied to both up- and down-type quark sector except for $y_{14}^{d}$ since quark doublet rotation is already used in the up-type quark sector. These two mass matrices clearly tells that this model is an extended 2HDM in that the up-type SM Higgs $H_{u}$ corresponds to up-type quark sector, while the down-type SM Higgs $H_{d}$ corresponds for downtype quark sector.

\section{A basis for decoupling heavy fourth and fifth vectorlike family}

In this section, we treat the decoupling basis with quarks holding an assumption $\langle\phi\rangle \approx M_{44}^{Q}$. As in the charged lepton mass matrix, we can obtain the Yukawa matrix from the $5 \times 5$ upper blocks of Eq. (A1),

$$
\tilde{y}_{\alpha \beta}^{u}=\left(\begin{array}{ccccc}
0 & 0 & 0 & 0 & y_{15}^{u} \\
0 & 0 & 0 & y_{24}^{u} & y_{25}^{u} \\
0 & 0 & 0 & y_{34}^{u} & y_{35}^{u} \\
0 & 0 & y_{43}^{u} & 0 & 0 \\
y_{51}^{u} & y_{52}^{u} & y_{53}^{u} & 0 & 0
\end{array}\right), \quad \tilde{y}_{\alpha \beta}^{d}=\left(\begin{array}{ccccc}
0 & 0 & 0 & y_{14}^{d} & y_{15}^{d} \\
0 & 0 & 0 & y_{24}^{d} & y_{25}^{d} \\
0 & 0 & 0 & y_{34}^{d} & y_{35}^{d} \\
0 & 0 & y_{43}^{d} & 0 & 0 \\
y_{51}^{d} & y_{52}^{d} & y_{53}^{d} & 0 & 0
\end{array}\right)
$$

where $\alpha$ and $\beta$ run from 1 to 5 . The Yukawa matrices $\tilde{y}_{\alpha \beta}^{u, d}$ can be diagonalized by the unitary rotations $V$

$$
V_{Q}=V_{45}^{Q} V_{35}^{Q} V_{25}^{Q} V_{15}^{Q} V_{34}^{Q} V_{24}^{Q} V_{14}^{Q}, \quad V_{u}=V_{45}^{u} V_{35}^{u} V_{25}^{u} V_{15}^{u} V_{34}^{u} V_{24}^{u} V_{14}^{u}, \quad V_{d}=V_{45}^{d} V_{35}^{d} V_{25}^{d} V_{15}^{d} V_{34}^{d} V_{24}^{d} V_{14}^{d}
$$

where each of the unitary matrices $V_{i 4,5}$ are parametrized by a single angle $\theta_{i 4,5}$ featuring the mixing between the $i$ th SM chiral quark and the 4,5th vectorlike quark. In the rotated mass matrix, we need $(3,4),(1,5),(2,5),(3,5)$ mixing in the $Q$ sector and $(2,4),(3,4),(1,5),(2,5),(3,5)$ mixing in the $u, d$ sectors to go to the decoupling basis therefore the unitary mixing matrices $V$ are defined to be 


$$
\begin{aligned}
& V_{Q}=V_{35}^{Q} V_{25}^{Q} V_{15}^{Q} V_{34}^{Q} \\
& =\left(\begin{array}{ccccc}
1 & 0 & 0 & 0 & 0 \\
0 & 1 & 0 & 0 & 0 \\
0 & 0 & c_{35}^{Q} & 0 & s_{35}^{Q} \\
0 & 0 & 0 & 1 & 0 \\
0 & 0 & -s_{35}^{Q} & 0 & c_{35}^{Q}
\end{array}\right)\left(\begin{array}{ccccc}
1 & 0 & 0 & 0 & 0 \\
0 & c_{25}^{Q} & 0 & 0 & s_{25}^{Q} \\
0 & 0 & 1 & 0 & 0 \\
0 & 0 & 0 & 1 & 0 \\
0 & -s_{25}^{Q} & 0 & 0 & c_{25}^{Q}
\end{array}\right)\left(\begin{array}{ccccc}
c_{15}^{Q} & 0 & 0 & 0 & s_{15}^{Q} \\
0 & 1 & 0 & 0 & 0 \\
0 & 0 & 1 & 0 & 0 \\
0 & 0 & 0 & 1 & 0 \\
-s_{15}^{Q} & 0 & 0 & 0 & c_{15}^{Q}
\end{array}\right)\left(\begin{array}{ccccc}
1 & 0 & 0 & 0 & 0 \\
0 & 1 & 0 & 0 & 0 \\
0 & 0 & c_{34}^{Q} & s_{34}^{Q} & 0 \\
0 & 0 & -s_{34}^{Q} & c_{34}^{Q} & 0 \\
0 & 0 & 0 & 0 & 1
\end{array}\right), \\
& \approx\left(\begin{array}{ccccc}
1 & 0 & 0 & 0 & s_{15}^{Q} \\
0 & 1 & 0 & 0 & s_{25}^{Q} \\
0 & 0 & 1 & s_{34}^{Q} & s_{35}^{Q} \\
0 & 0 & -s_{34}^{Q} & 1 & 0 \\
-s_{15}^{Q} & -s_{25}^{Q} & -s_{15}^{Q} & 0 & 1
\end{array}\right) \\
& s_{34}^{Q}=\frac{x_{34}^{Q}\langle\phi\rangle}{\sqrt{\left(x_{34}^{Q}\langle\phi\rangle\right)^{2}+\left(M_{44}^{Q}\right)^{2}}}, \quad s_{15}^{Q}=\frac{x_{15}^{Q}\langle\phi\rangle}{\sqrt{\left(x_{15}^{Q}\langle\phi\rangle\right)^{2}+\left(M_{55}^{Q}\right)^{2}}}, \\
& s_{25}^{Q}=\frac{x_{25}^{Q}\langle\phi\rangle}{\sqrt{\left(x_{25}^{Q}\langle\phi\rangle\right)^{2}+\left(M_{55}^{\prime Q}\right)^{2}}}, \quad s_{35}^{Q}=\frac{x_{35}^{\prime Q}\langle\phi\rangle}{\sqrt{\left(x_{35}^{\prime Q}\langle\phi\rangle\right)^{2}+\left(M_{55}^{\prime \prime Q}\right)^{2}}}, \\
& x_{35}^{\prime Q}\langle\phi\rangle=c_{34}^{Q} x_{35}^{Q}\langle\phi\rangle+s_{34}^{Q} M_{45}^{Q}, \quad M_{45}^{\prime Q}=-s_{34}^{Q} x_{35}^{Q}\langle\phi\rangle+c_{34}^{Q} M_{45}^{Q} \\
& \tilde{M}_{44}^{Q}=\sqrt{\left(x_{34}^{Q}\langle\phi\rangle\right)^{2}+\left(M_{44}^{Q}\right)^{2}}, \\
& M_{55}^{\prime Q}=\sqrt{\left(x_{15}^{Q}\langle\phi\rangle\right)^{2}+\left(M_{55}^{Q}\right)^{2}}, \quad M_{55}^{\prime \prime Q}=\sqrt{\left(x_{25}^{Q}\langle\phi\rangle\right)^{2}+\left(M_{55}^{\prime Q}\right)^{2}}, \quad \tilde{M}_{55}^{Q}=\sqrt{\left(x_{35}^{\prime Q}\langle\phi\rangle\right)^{2}+\left(M_{55}^{\prime \prime Q}\right)^{2}} \\
& V_{u}=V_{35}^{u} V_{25}^{u} V_{15}^{u} V_{34}^{u} V_{24}^{u} \\
& =\left(\begin{array}{ccccc}
1 & 0 & 0 & 0 & 0 \\
0 & 1 & 0 & 0 & 0 \\
0 & 0 & c_{35}^{u} & 0 & s_{35}^{u} \\
0 & 0 & 0 & 1 & 0 \\
0 & 0 & -s_{35}^{u} & 0 & c_{35}^{u}
\end{array}\right)\left(\begin{array}{ccccc}
1 & 0 & 0 & 0 & 0 \\
0 & c_{25}^{u} & 0 & 0 & s_{25}^{u} \\
0 & 0 & 1 & 0 & 0 \\
0 & 0 & 0 & 1 & 0 \\
0 & -s_{25}^{u} & 0 & 0 & c_{25}^{u}
\end{array}\right)\left(\begin{array}{ccccc}
c_{15}^{u} & 0 & 0 & 0 & s_{15}^{u} \\
0 & 1 & 0 & 0 & 0 \\
0 & 0 & 1 & 0 & 0 \\
0 & 0 & 0 & 1 & 0 \\
-s_{15}^{u} & 0 & 0 & 0 & c_{15}^{u}
\end{array}\right) \\
& \times\left(\begin{array}{ccccc}
1 & 0 & 0 & 0 & 0 \\
0 & 1 & 0 & 0 & 0 \\
0 & 0 & c_{34}^{u} & s_{34}^{u} & 0 \\
0 & 0 & -s_{34}^{u} & c_{34}^{u} & 0 \\
0 & 0 & 0 & 0 & 1
\end{array}\right)\left(\begin{array}{ccccc}
1 & 0 & 0 & 0 & 0 \\
0 & c_{24}^{u} & 0 & s_{24}^{u} & 0 \\
0 & 0 & 1 & 0 & 0 \\
0 & -s_{24}^{u} & 0 & c_{24}^{u} & 0 \\
0 & 0 & 0 & 0 & 1
\end{array}\right) \approx\left(\begin{array}{ccccc}
1 & 0 & 0 & 0 & \theta_{15}^{u} \\
0 & 1 & 0 & \theta_{24}^{u} & \theta_{25}^{u} \\
0 & 0 & 1 & \theta_{34}^{u} & \theta_{35}^{u} \\
0 & -\theta_{24}^{u} & -\theta_{34}^{u} & 1 & 0 \\
-\theta_{15}^{u} & -\theta_{25}^{u} & -\theta_{35}^{u} & 0 & 1
\end{array}\right), \\
& s_{24}^{u} \approx \frac{x_{42}^{u}\langle\phi\rangle}{M_{44}^{u}}, \quad s_{34}^{u} \approx \frac{x_{43}^{u}\langle\phi\rangle}{M_{44}^{\prime u}}, \quad s_{15}^{u} \approx \frac{x_{51}^{u}\langle\phi\rangle}{M_{55}^{u}}, \quad s_{25}^{u} \approx \frac{x_{52}^{\prime u}\langle\phi\rangle}{M_{55}^{\prime u}}, \quad s_{35}^{u} \approx \frac{x_{53}^{u}\langle\phi\rangle}{M_{55}^{\prime u}}, \\
& x_{52}^{\prime u}\langle\phi\rangle=c_{24}^{u} x_{52}^{u}\langle\phi\rangle+s_{24}^{u} M_{54}^{u}, \quad M_{54}^{\prime u}=-s_{24}^{u} x_{52}^{u}\langle\phi\rangle+c_{24}^{u} M_{54}^{u}, \\
& x_{53}^{\prime u}\langle\phi\rangle=c_{34}^{u} x_{53}^{u}\langle\phi\rangle+s_{34}^{u} M_{54}^{\prime u}, \quad M_{54}^{\prime \prime u}=-s_{34}^{u} x_{53}^{u}\langle\phi\rangle+c_{34}^{u} M_{54}^{\prime u} \text {, } \\
& M_{44}^{\prime u}=\sqrt{\left(x_{42}^{u}\langle\phi\rangle\right)^{2}+\left(M_{44}^{u}\right)^{2}}, \quad \tilde{M}_{44}^{u}=\sqrt{\left(x_{43}^{u}\langle\phi\rangle\right)^{2}+\left(M_{44}^{u}\right)^{2}}, \\
& M_{55}^{\prime \prime}=\sqrt{\left(x_{51}^{u}\langle\phi\rangle\right)^{2}+\left(M_{55}^{u}\right)^{2}}, \quad M_{55}^{\prime \prime u}=\sqrt{\left(x_{52}^{\prime \prime}\langle\phi\rangle\right)^{2}+\left(M_{55}^{\prime \prime}\right)^{2}}, \quad \tilde{M}_{55}^{u}=\sqrt{\left(x_{53}^{\prime \prime}\langle\phi\rangle\right)^{2}+\left(M_{55}^{\prime \prime u}\right)^{2}} .
\end{aligned}
$$


With the defined unitary mixing matrices in place, the $5 \times 5$ Yukawa matrices in a mass basis (primed) are transformed by

$$
\tilde{y}_{\alpha \beta}^{\prime u}=V_{Q} \tilde{y}_{\alpha \beta}^{u} V_{u}^{\dagger}, \quad \tilde{y}_{\alpha \beta}^{d d}=V_{Q} \tilde{y}_{\alpha \beta}^{d} V_{d}^{\dagger},
$$

where tilde with prime means interaction basis whereas tilde alone corresponds to the mass basis. The effective SM
Yukawa couplings for the quarks then correspond to the $3 \times 3$ upper block of $\tilde{y}_{\alpha \beta}^{\prime \prime}, \tilde{y}_{\alpha \beta}^{\prime d}$, namely

$y_{i j}^{u} \tilde{H}_{u} \bar{Q}_{i L} u_{j R}, \quad y_{i j}^{d} \tilde{H}_{d} \bar{Q}_{i L} d_{j R}, \quad$ with $\quad y_{i j}^{u} \equiv \tilde{y}_{i j}^{\prime u}$,

$y_{i j}^{d} \equiv \tilde{y}_{i j}^{\prime d}, \quad(i, \quad j=1,2,3)$.

The $3 \times 3$ SM Yukawa matrices for up- and down-type quark sector read:

$$
\begin{array}{r}
y_{i j}^{u}=\left(\begin{array}{ccc}
s_{15}^{Q} y_{51}^{u}+y_{15}^{u} \theta_{15}^{u} & s_{15}^{Q} y_{52}^{u}+y_{15}^{u} \theta_{25}^{u} & s_{15}^{Q} y_{53}^{u}+y_{15}^{u} \theta_{35}^{u} \\
s_{25}^{Q} y_{51}^{u}+y_{25}^{u} \theta_{15}^{u} & s_{25}^{Q} y_{52}^{u}+y_{24}^{u} \theta_{24}^{u}+y_{25}^{u} \theta_{25}^{u} & s_{25}^{Q} y_{53}^{u}+y_{24}^{u} \theta_{34}^{u}+y_{25}^{u} \theta_{35}^{u} \\
s_{35}^{Q} y_{51}^{u}+y_{35}^{u} \theta_{15}^{u} & s_{35}^{Q} y_{52}^{u}+y_{34}^{u} \theta_{24}^{u}+y_{35}^{u} \theta_{25}^{u} & s_{34}^{Q} y_{43}^{u}+s_{35}^{Q} y_{53}^{u}+y_{34}^{u} \theta_{34}^{u}+y_{35}^{u} \theta_{35}^{u}
\end{array}\right) \\
y_{i j}^{d}=\left(\begin{array}{ccc}
s_{15}^{Q} y_{51}^{d}+y_{15}^{d} \theta_{15}^{d} & s_{15}^{Q} y_{52}^{d}+y_{14}^{d} \theta_{24}^{d}+y_{15}^{d} \theta_{25}^{d} & s_{15}^{Q} y_{53}^{d}+y_{14}^{d} \theta_{34}^{d}+y_{15}^{d} \theta_{35}^{d} \\
s_{25}^{Q} y_{51}^{d}+y_{25}^{d} \theta_{15}^{d} & s_{25}^{Q} y_{52}^{d}+y_{24}^{d} \theta_{24}^{d}+y_{25}^{d} \theta_{25}^{d} & s_{25}^{Q} y_{53}^{d}+y_{24}^{d} \theta_{34}^{d}+y_{25}^{d} \theta_{35}^{d} \\
s_{35}^{Q} y_{51}^{d}+y_{35}^{d} \theta_{15}^{d} & s_{35}^{Q} y_{52}^{d}+y_{34}^{d} \theta_{24}^{d}+y_{35}^{d} \theta_{25}^{d} & s_{34}^{Q} y_{43}^{d}+s_{35}^{Q} y_{53}^{d}+y_{34}^{d} \theta_{34}^{d}+y_{35}^{d} \theta_{35}^{d}
\end{array}\right)
\end{array}
$$

\section{APPENDIX B: HEAVY SCALAR PRODUCTION AT A PROTON-PROTON COLLIDER}

We have confirmed that the mass of the non-SM $C P$ even scalar $H_{1}$ is ranged from 200 to $240 \mathrm{GeV}$ in Table IV and this light mass of $H_{1}$ has not been observed at CERN or other experiments so far. In order to see how big an impact of $H_{1}$ is when compared to that of SM Higgs $h$, we studied a total cross section for the SM process $p p \rightarrow h$ and for BSM process $p p \rightarrow H_{1}$. The SM cross section for $p p \rightarrow h$ process is

$$
\begin{aligned}
\sigma_{\mathrm{SM}}= & \frac{\alpha_{S}^{2} m_{h}^{2}}{64 \pi v^{2}}\left(L\left(\frac{m_{h}^{2}}{m_{t}^{2}}\right)\right)^{2} \\
& \times \frac{1}{S} \int_{\ln \sqrt{m_{h}^{2} / S}}^{-\ln \sqrt{m_{h}^{2} / S}} \operatorname{PDF}\left(0, x_{1}(y), m_{h}\right) \\
& \times P D F\left(0, x_{2}(y), m_{h}\right) d y
\end{aligned}
$$

where $L$ is a loop integral

$$
\begin{aligned}
L(a)= & \mid[2 a+(-4+a) \operatorname{Poly} \log (2,1 / 2(-\sqrt{-4+a} \sqrt{a}+a)) \\
& +(-4+a) \operatorname{PolyLog}(2,1 / 2(\sqrt{-4+a} \sqrt{a}+a))] / a^{2} \mid,
\end{aligned}
$$

$\alpha_{S}$ is the strong coupling constant, $v$ is the conventional SM Higgs vev $246.22 \mathrm{GeV}, m_{h}$ is the Higgs mass $125 \mathrm{GeV}$, $m_{t}$ is the top quark mass $173 \mathrm{GeV}, S$ is the squared LHC center of mass energy $(14 \mathrm{TeV})^{2}, P D F$ corresponds to the parton distribution function where 0 means 0 th partongluon, $x$ is the momentum fraction of the proton carried out by the gluon. Here the factorization scale has been taken to be equal to the SM like Higgs boson mass $m_{h}$ and $x_{1,2}(y)$ are defined as follows:

$$
\begin{aligned}
& x_{1}(y)=\frac{\sqrt{m_{h}^{2} / S}}{S} \exp (y), \\
& x_{2}(y)=\frac{\sqrt{m_{h}^{2} / S}}{S} \exp (-y) .
\end{aligned}
$$

With these defined functions and values, the total cross section for $p p \rightarrow h$ is

$$
\sigma_{\mathrm{SM}} \simeq 18 p b .
$$

Next, the total cross section for $p p \rightarrow H_{1}$ process is

$$
\begin{aligned}
\sigma\left(p p \rightarrow H_{1}\right)= & \frac{\alpha_{S}^{2} m_{H_{1}}^{2} a_{h b b}^{2}}{64 \pi v_{2}^{2}}\left(L\left(\frac{m_{H_{1}}^{2}}{m_{b}^{2}}\right)\right)^{2} \\
& \times \frac{1}{S} \int_{\ln \sqrt{m_{H_{1}}^{2} / S}}^{-\ln \sqrt{m_{H_{1}}^{2} / S}} \operatorname{PDF}\left(0, x_{1}^{\prime}(y), m_{H_{1}}\right) \\
& \times P D F\left(0, x_{2}^{\prime}(y), m_{H_{1}}\right) d y
\end{aligned}
$$

where $m_{H_{1}}$ is mass of non-SM $C P$ even scalar $H_{1}$, and $x_{1,2}^{\prime}$ are defined in a similar way:

$$
\begin{aligned}
& x_{1}^{\prime}(y)=\frac{\sqrt{m_{H_{1}}^{2} / S}}{S} \exp (y), \\
& x_{2}^{\prime}(y)=\frac{\sqrt{m_{H_{1}}^{2} / S}}{S} \exp (-y)
\end{aligned}
$$

One main distinction between Eqs. (B1) and (B5) is the non-SM scalar $H_{1}$ only interacts with down-type quark pair 
$b \bar{b}$ since it is a mixed state between $h_{d}^{0}$ and $\phi$ while the SM Higgs $h$ can interact with top-quark pair $t \bar{t}$. According to the mass range of $H_{1}$ reported in Table IV, the total cross section for $p p \rightarrow H_{1}$ is given in Fig. 13.

The total cross section for $p p \rightarrow H_{1}$ runs from nearly $8 p b$ at $200 \mathrm{GeV}$ to smaller values as mass of $H_{1}$ increases. The order of magnitude of this cross section for $p p \rightarrow H_{1}$ is compatible to that of the SM process $p p \rightarrow h$, however the BSM process is strongly suppressed since its single LHC production via gluon fusion mechanism is dominated by the triangular bottom quark loop as mentioned in Sec. VI. Therefore, our prediction with the light non-SM scalar $H_{1}$ is possible to accommodate each anomaly constraint at $1 \sigma$.

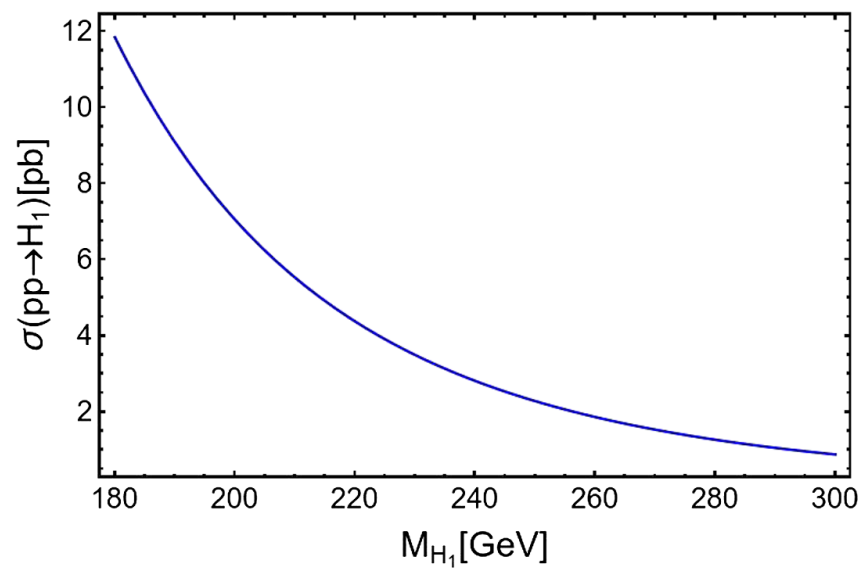

FIG. 13. The total cross section for $p p \rightarrow H_{1}$ at $14 \mathrm{TeV}$.
[1] G. W. Bennett et al. (Muon g-2 Collaboration), Phys. Rev. D 73, 072003 (2006).

[2] K. Hagiwara, R. Liao, A. D. Martin, D. Nomura, and T. Teubner, J. Phys. G 38, 085003 (2011).

[3] M. Davier, A. Hoecker, B. Malaescu, and Z. Zhang, Eur. Phys. J. C 77, 827 (2017).

[4] T. Blum, P. A. Boyle, V. Gülpers, T. Izubuchi, L. Jin, C. Jung, A. Jüttner, C. Lehner, A. Portelli, and J. T. Tsang (RBC and UKQCD Collaborations), Phys. Rev. Lett. 121, 022003 (2018).

[5] A. Keshavarzi, D. Nomura, and T. Teubner, Phys. Rev. D 97, 114025 (2018).

[6] T. Aoyama, N. Asmussen, M. Benayoun, J. Bijnens, T. Blum, M. Bruno, I. Caprini, C. M. Carloni Calame, M. Cè, and G. Colangelo et al., Phys. Rep. 887, 1 (2020).

[7] R. H. Parker, C. Yu, W. Zhong, B. Estey, and H. Müller, Science 360, 191 (2018).

[8] L. Morel, Z. Yao, P. Cladé, and S. Guellati-Khélifa, Nature (London) 588, 61 (2020).

[9] S. F. King, J. High Energy Phys. 09 (2018) 069.

[10] S. Centelles Chuliá, C. Döring, W. Rodejohann, and U. J. Saldaña-Salazar, J. High Energy Phys. 09 (2020) 137.

[11] J. Hernandez-Garcia and S. F. King, J. High Energy Phys. 05 (2019) 169.

[12] A. Crivellin, M. Hoferichter, and P. Schmidt-Wellenburg, Phys. Rev. D 98, 113002 (2018).

[13] A. Freitas, J. Lykken, S. Kell, and S. Westhoff, J. High Energy Phys. 05 (2014) 145; 09 (2014) 155(E).

[14] T. Appelquist, M. Piai, and R. Shrock, Phys. Lett. B 593, 175 (2004).

[15] G. F. Giudice, P. Paradisi, and M. Passera, J. High Energy Phys. 11 (2012) 113.

[16] A. Falkowski, S. F. King, E. Perdomo, and M. Pierre, J. High Energy Phys. 08 (2018) 061.

[17] B. Allanach, F. S. Queiroz, A. Strumia, and S. Sun, Phys. Rev. D 93, 055045 (2016); 95, 119902(E) (2017).
[18] C. H. Chen, T. Nomura, and H. Okada, Phys. Rev. D 94, 115005 (2016).

[19] S. Raby and A. Trautner, Phys. Rev. D 97, 095006 (2018).

[20] C. W. Chiang, H. Okada, and E. Senaha, Phys. Rev. D 96, 015002 (2017).

[21] C. H. Chen, T. Nomura, and H. Okada, Phys. Lett. B 774, 456 (2017).

[22] H. Davoudiasl and W. J. Marciano, Phys. Rev. D 98, 075011 (2018).

[23] J. Liu, C. E. M. Wagner, and X. P. Wang, J. High Energy Phys. 03 (2019) 008.

[24] A. E. Cárcamo Hernández, S. Kovalenko, R. Pasechnik, and I. Schmidt, Eur. Phys. J. C 79, 610 (2019).

[25] T. Nomura and H. Okada, Phys. Rev. D 101, 015021 (2020).

[26] J. Kawamura, S. Raby, and A. Trautner, Phys. Rev. D 100, 055030 (2019).

[27] M. Bauer, M. Neubert, S. Renner, M. Schnubel, and A. Thamm, Phys. Rev. Lett. 124, 211803 (2020).

[28] X. F. Han, T. Li, L. Wang, and Y. Zhang, Phys. Rev. D 99, 095034 (2019).

[29] B. Dutta and Y. Mimura, Phys. Lett. B 790, 563 (2019).

[30] M. Badziak and K. Sakurai, J. High Energy Phys. 10 (2019) 024.

[31] M. Endo and W. Yin, J. High Energy Phys. 08 (2019) 122.

[32] G. Hiller, C. Hormigos-Feliu, D. F. Litim, and T. Steudtner, Phys. Rev. D 102, 071901 (2020).

[33] A. E. Cárcamo Hernández, S. F. King, H. Lee, and S. J. Rowley, Phys. Rev. D 101, 115016 (2020).

[34] A. E. Cárcamo Hernández, D. T. Huong, and H. N. Long, Phys. Rev. D 102, 055002 (2020).

[35] J. Kawamura, S. Raby, and A. Trautner, Phys. Rev. D 101, 035026 (2020).

[36] C. Cornella, P. Paradisi, and O. Sumensari, J. High Energy Phys. 01 (2020) 158.

[37] A. E. Cárcamo Hernández, Y. Hidalgo Velásquez, S. Kovalenko, H. N. Long, N. A. Pérez-Julve, and V. V. Vien, Eur. Phys. J. C 81, 191 (2021). 
[38] C. Arbeláez, R. Cepedello, R. M. Fonseca, and M. Hirsch, Phys. Rev. D 102, 075005 (2020).

[39] G. Hiller, C. Hormigos-Feliu, D. F. Litim, and T. Steudtner, Phys. Rev. D 102, 095023 (2020).

[40] S. Jana, V. P. K., and S. Saad, Phys. Rev. D 101, 115037 (2020).

[41] Á. S. de Jesus, S. Kovalenko, C. A. de S. Pires, F. S. Queiroz, and Y.S. Villamizar, Phys. Lett. B 809, 135689 (2020).

[42] A. S. De Jesus, S. Kovalenko, F. S. Queiroz, C. Siqueira, and K. Sinha, Phys. Rev. D 102, 035004 (2020).

[43] C. Hati, J. Kriewald, J. Orloff, and A. M. Teixeira, J. High Energy Phys. 07 (2020) 235.

[44] F. J. Botella, F. Cornet-Gomez, and M. Nebot, Phys. Rev. D 102, 035023 (2020).

[45] I. Doršner, S. Fajfer, and S. Saad, Phys. Rev. D 102, 075007 (2020).

[46] L. Calibbi, M. L. López-Ibáñez, A. Melis, and O. Vives, J. High Energy Phys. 06 (2020) 087.

[47] L. T. Hue, P. N. Thanh, and T. D. Tham, Commun. Phys. 30, 221 (2020).

[48] S. Jana, P. K. Vishnu, W. Rodejohann, and S. Saad, Phys. Rev. D 102, 075003 (2020).

[49] E. J. Chun and T. Mondal, J. High Energy Phys. 11 (2020) 077.

[50] C. K. Chua, Phys. Rev. D 102, 055022 (2020).

[51] Y. Daikoku and H. Okada, arXiv:2011.10374.

[52] H. Banerjee, B. Dutta, and S. Roy, J. High Energy Phys. 03 (2021) 211.

[53] C. H. Chen and T. Nomura, Nucl. Phys. B964, 115314 (2021).

[54] I. Bigaran and R. R. Volkas, Phys. Rev. D 102, 075037 (2020).

[55] J. Kawamura, S. Okawa, and Y. Omura, J. High Energy Phys. 08 (2020) 042.

[56] M. Endo, S. Iguro, and T. Kitahara, J. High Energy Phys. 06 (2020) 040.

[57] N. Chakrabarty, arXiv:2010.05215.

[58] S. P. Li, X. Q. Li, Y. Y. Li, Y. D. Yang, and X. Zhang, J. High Energy Phys. 01 (2021) 034.

[59] A. Davidson and K.C. Wali, Phys. Rev. Lett. 59, 393 (1987).

[60] A. Davidson and K. C. Wali, Phys. Rev. Lett. 58, 2623 (1987).

[61] Z. G. Berezhiani and R. Rattazzi, Phys. Lett. B 279, 124 (1992).

[62] I. S. Sogami and T. Shinohara, Prog. Theor. Phys. 86, 1031 (1991).

[63] P. H. Gu and M. Lindner, Phys. Lett. B 698, 40 (2011).

[64] C. Alvarado, R. Martinez, and F. Ochoa, Phys. Rev. D 86, 025027 (2012).

[65] A. E. Carcamo Hernandez, R. Martinez, and F. Ochoa, Phys. Rev. D 87, 075009 (2013).

[66] R. Kawasaki, T. Morozumi, and H. Umeeda, Phys. Rev. D 88, 033019 (2013).

[67] R. N. Mohapatra and Y. Zhang, J. High Energy Phys. 06 (2014) 072.
[68] P. S. B. Dev, R. N. Mohapatra, and Y. Zhang, J. High Energy Phys. 02 (2016) 186.

[69] D. Borah and S. Patra, Phys. Lett. B 771, 318 (2017).

[70] A. Patra and S. K. Rai, Phys. Rev. D 98, 015033 (2018).

[71] K. S. Babu, B. Dutta, and R. N. Mohapatra, J. High Energy Phys. 01 (2019) 168.

[72] I. de Medeiros Varzielas and S. F. King, J. High Energy Phys. 11 (2018) 100.

[73] A. E. Cárcamo Hernández and S. F. King, Phys. Rev. D 99, 095003 (2019).

[74] A. E. Cárcamo Hernández, Y. Hidalgo Velásquez, and N. A. Pérez-Julve, Eur. Phys. J. C 79, 828 (2019).

[75] A. E. Cárcamo Hernández, J. Marchant González, and U. J. Saldaña-Salazar, Phys. Rev. D 100, 035024 (2019).

[76] A. E. C. Hernández and I. Schmidt, arXiv:2101.02718.

[77] S. F. King, J. High Energy Phys. 08 (2017) 019.

[78] M. Blennow and E. Fernandez-Martinez, Phys. Lett. B 704, 223 (2011).

[79] L. L. Chau and W. Y. Keung, Phys. Rev. Lett. 53, 1802 (1984).

[80] E. Fernandez-Martinez, M. B. Gavela, J. Lopez-Pavon, and O. Yasuda, Phys. Lett. B 649, 427 (2007).

[81] A. Broncano, M. B. Gavela, and E. E. Jenkins, Phys. Lett. B 552, 177 (2003); 636, 332(E) (2006).

[82] L. Calibbi and G. Signorelli, Riv. Nuovo Cimento 41, 71 (2018).

[83] S. L. Glashow, J. Iliopoulos, and L. Maiani, Phys. Rev. D 2 , 1285 (1970).

[84] T. P. Cheng and L. F. Li, Gauge Theory Of Elementary Particle Physics (Clarendon, Oxford Science Publications, Oxford, United Kingdom, 1984), p. 536.

[85] M. Lindner, M. Platscher, and F. S. Queiroz, Phys. Rep. 731, 1 (2018).

[86] E. Fernandez-Martinez, J. Hernandez-Garcia, and J. Lopez-Pavon, J. High Energy Phys. 08 (2016) 033.

[87] M. Tanabashi et al. (Particle Data Group), Phys. Rev. D 98, 030001 (2018).

[88] A. M. Sirunyan et al. (CMS Collaboration), J. High Energy Phys. 11 (2018) 185.

[89] G. Aad et al. (ATLAS Collaboration), Phys. Rev. D 101, 012002 (2020).

[90] R. A. Diaz, R. Martinez, and J. A. Rodriguez, Phys. Rev. D 67, 075011 (2003).

[91] F. Jegerlehner and A. Nyffeler, Phys. Rep. 477, 1 (2009).

[92] C. Kelso, H. N. Long, R. Martinez, and F. S. Queiroz, Phys. Rev. D 90, 113011 (2014).

[93] K. Kowalska and E. M. Sessolo, J. High Energy Phys. 09 (2017) 112.

[94] F. Z. Xu, W. Zhang, J. Li, and T. Li, Phys. Rev. D 98, 115033 (2018).

[95] J. Hernández-Sánchez, C. G. Honorato, S. Moretti, and S. Rosado-Navarro, Phys. Rev. D 102, 055008 (2020).

[96] G. Bhattacharyya and D. Das, Pramana 87, 40 (2016).

[97] M. Maniatis, A. von Manteuffel, O. Nachtmann, and F. Nagel, Eur. Phys. J. C 48, 805 (2006). 